\title{
Alpha-synuclein aggregation: visualization by X-ray techniques and its modulation by iron
}

\author{
Dissertation \\ for the award of the degree \\ "Doctor of Philosophy" \\ Division of Mathematics and Natural Sciences
}

of the Georg-August-Universität Göttingen

within the doctoral program "Molecular physiology of the brain"

of the Georg-August University School of Science (GAUSS)

submitted by

Eleonora Carboni

From Urbino (Italy)

Göttingen, September 2016 



\section{Thesis Committee}

Prof. Dr. Paul Lingor (Department of Neurology, University Medical Center Göttingen)

Prof. Dr. Tim Salditt (Institute for X-ray physics, Faculty of Physics University of Göttingen)

Prof. Dr. Tiago Fleming Outeiro (NeuroDegeneration and Restaurative Research, University Medical Center Göttingen)

\section{Members of the Examination Board}

$\mathbf{1}^{\text {st }}$ Referee: Prof. Dr. Paul Lingor (Department of Neurology, University Medical Center Göttingen)

$2^{\text {nd }}$ Referee: Prof. Dr. Tim Salditt (Institute for X-ray physics, Faculty of Physics University of Göttingen)

Prof. Dr. Tiago Fleming Outeiro (NeuroDegeneration and Restaurative Research, University Medical Center Göttingen)

Prof. Dr. Matthias Bähr (Department of Neurology, University Medical Center Göttingen)

Dr. Kamin Dean (Trans-synaptic Signaling, European Neuroscience Institute Göttingen)

Dr. Sebastian Kügler (Department of Neurology, University Medical Center Göttingen)

Date of oral examination: 19.10.2016 


\section{Declaration}

I hereby declare that the thesis:

"Alpha-synuclein aggregation: visualization by $x$-ray techniques and its modulation by iron"

has been written independently and with no other sources and aids than quoted.

Eleonora Carboni

Göttingen, September 2016 

Considerate la vostra semenza:

fatti non foste a viver come bruti, ma per seguir virtute e conoscenza.

Denkt euren Ursprungs und aus welchem Grunde Ihr seid geboren, nicht wie Vieh zu leben, Nein! zu der Tugend und der Weisheit Funde.

Consider well the seed that gave you birth: you were not made to live as brutes, but to follow virtue and knowledge.

Dante (1265-1321)

Divina Commedia (Inferno, XXVI vv118-120) 


\section{Table of Contents}

Table of Contents i

List of the figures. iv

Abbreviation List ...

1.

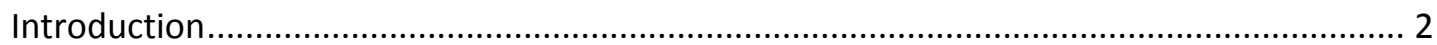

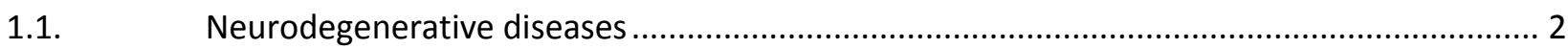

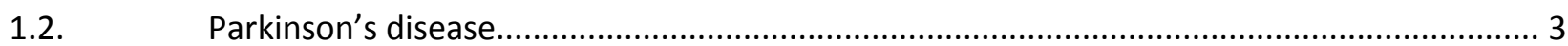

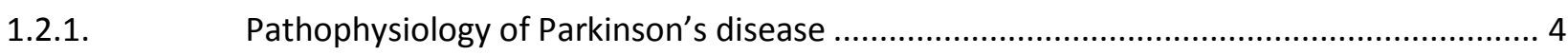

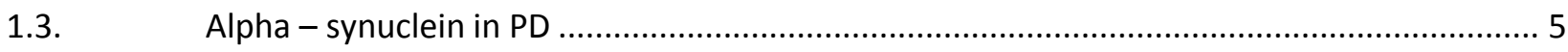

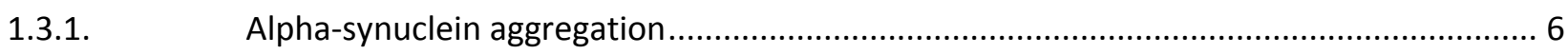

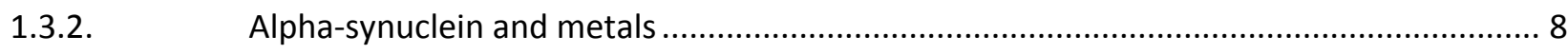

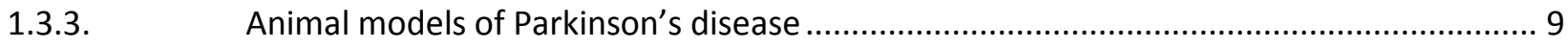

1.3.4. Use of chelators in neurodegeneration and Parkinson's disease ................................... 12

1.4. $\quad \mathrm{X}$ - ray imaging techniques and their principles ......................................................... 13

1.4.1. X-ray fluorescence and particle induced X-ray emission: principles and uses ................ 15

1.4.2. $\quad X$ ray scattering- based imaging techniques: SAXS, WAXS and STXM.......................... 17

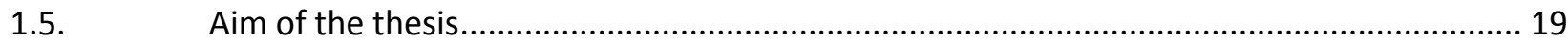

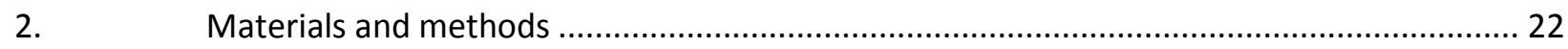

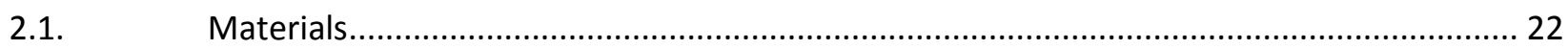

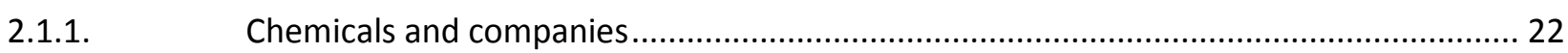

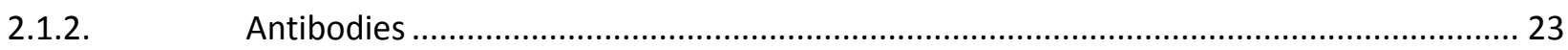

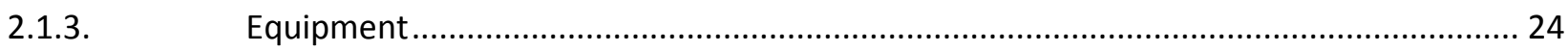

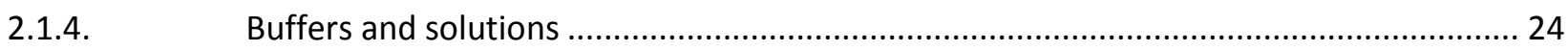

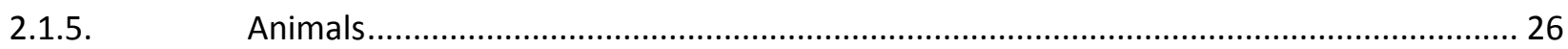

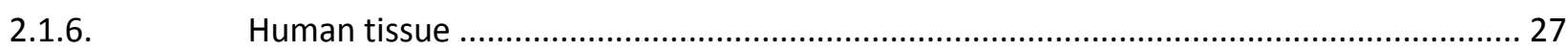

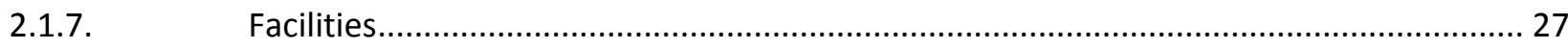

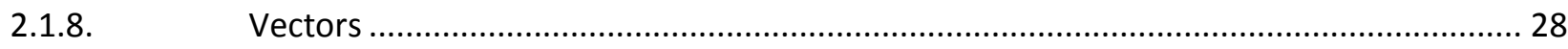

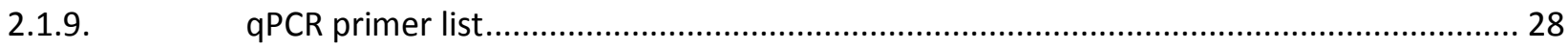

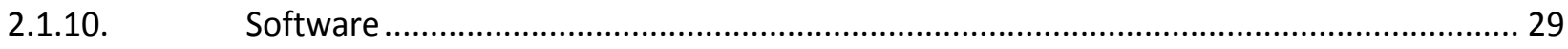

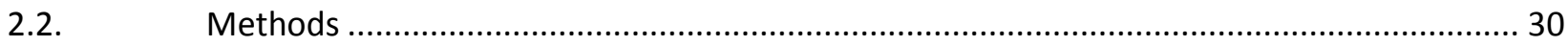

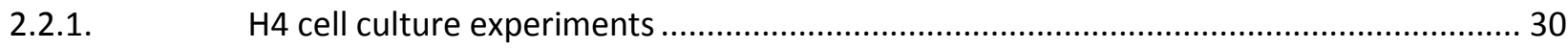

2.2.2. Tissue preparation for $\mathrm{X}$-ray diffraction and $\mathrm{X}$-ray fluorescence and analysis ................ 31

2.2.3. Tissue preparation for particle induced $X$-ray emission ............................................... 34 
2.2.4. Primary midbrain neurons culture preparation for particle induced $\mathrm{x}$-ray

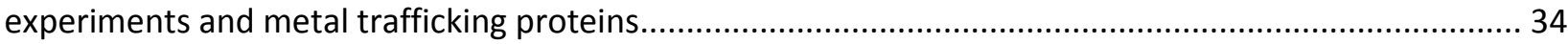

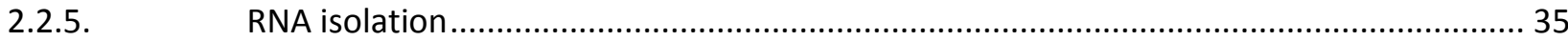

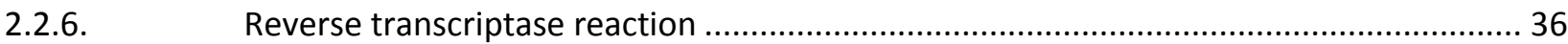

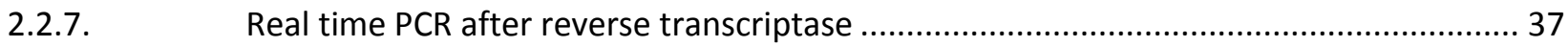

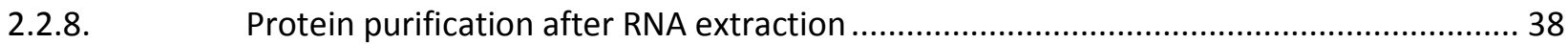

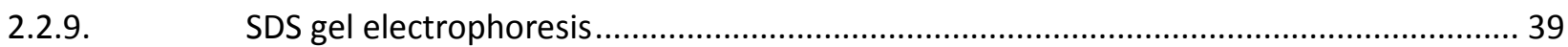

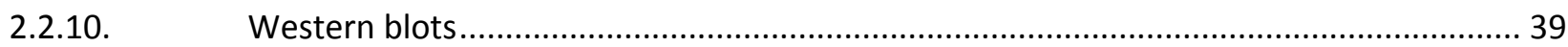

2.2.11. Iron intoxication protocol and chelator treatment..................................................... 41

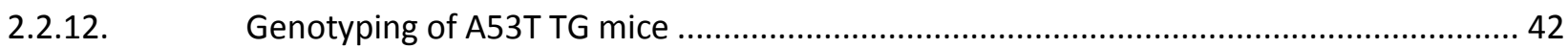

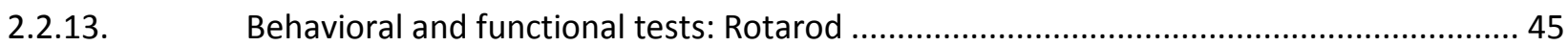

2.2.14. Behavioral and functional tests: Novel object recognition (NOR) test.......................... 47

2.2.15. Behavioral and functional tests: Catwalk.................................................................... 48

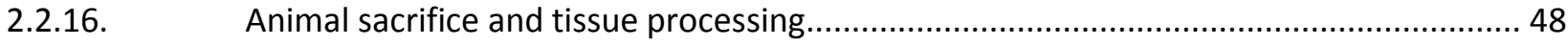

2.2.17. Proteinase K digestions and dot blots........................................................................ 49

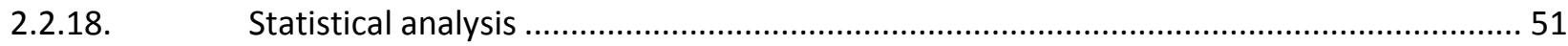

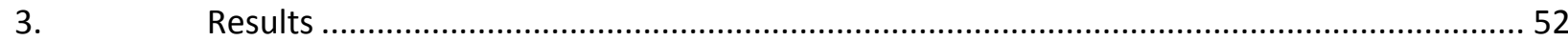

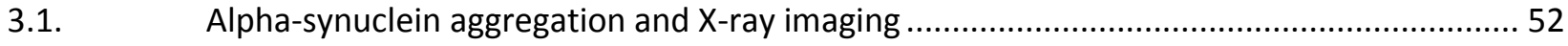

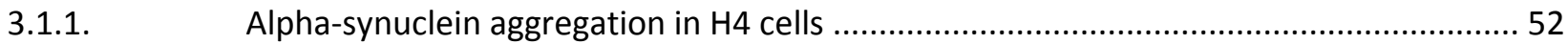

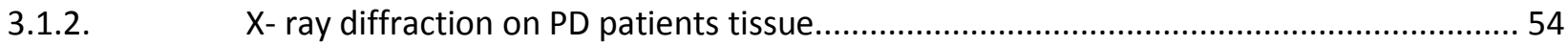

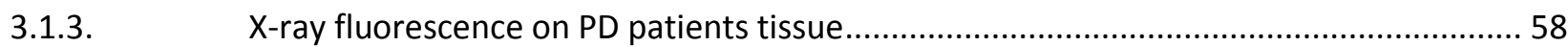

3.1.4. Particle induced X-ray emission on PD patient tissue.................................................. 62

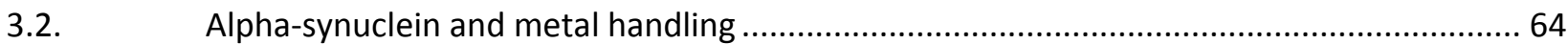

3.2.1. Alpha synuclein overexpression and Fe supplementation in primary neuronal cultures $\quad 65$

3.2.2. Alpha-synuclein overexpression and metal transporting proteins............................... 68

3.3. In-vivo aggregation study: A53T mice and Fe supply ..................................................... 71

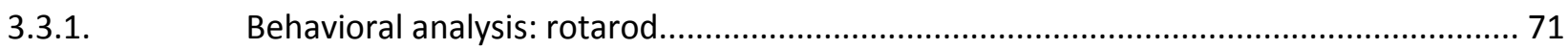

3.3.2. Behavioral analysis: Novel object recognition (NOR) ................................................ 73

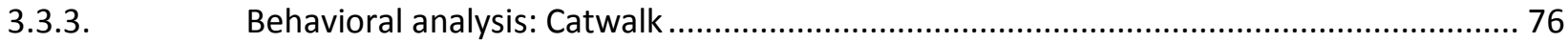

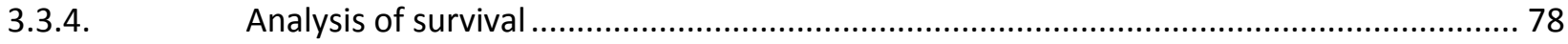

3.3.5. Biochemical characterization of the aggregation ......................................................... 79

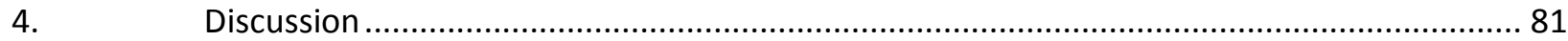

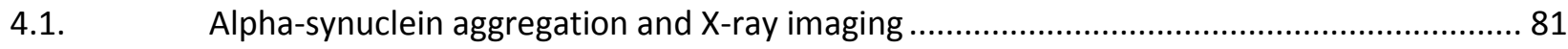

4.1.1. Aggregates in $\mathrm{H} 4$ cells do not give a signal in STXM .................................................. 82 
4.1.2. PD patients tissue shows characteristic peaks in the diffraction patterns at wide angles $\quad 84$

4.1.3. $\quad \mathrm{Cu}$ and Fe quantification in PD patient's tissue ......................................................... 87

4.1.4. Particle induced X-ray emission on PD patient tissue reveals lower concentration

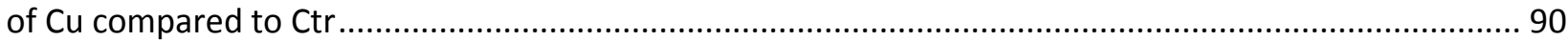

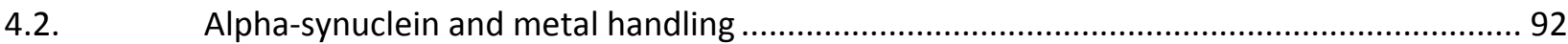

4.2.1. Alpha synuclein overexpression and Fe supply cause elemental dys-homeostasis in

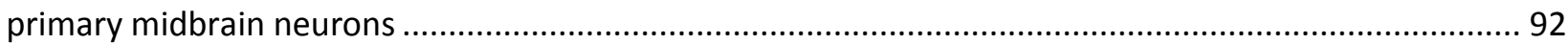

4.2.2. Alpha synuclein down-regulates CTR1 levels............................................................. 94

4.3. In-vivo aggregation study: A53T mice and Fe supply ...................................................... 95

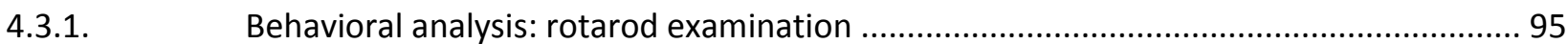

4.3.2. Behavioral analysis: Novel object recognition ............................................................. 96

4.3.3. Behavioral analysis: Catwalk analysis ......................................................................... 97

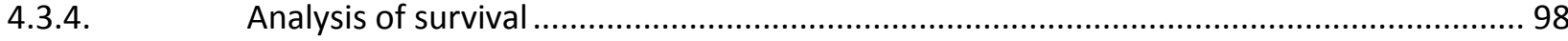

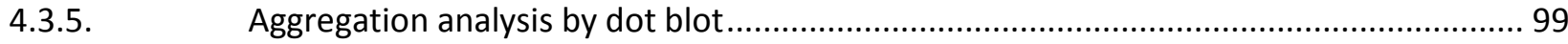

4.3.6. A53T mice and Fe intoxication in perspective ........................................................ 100

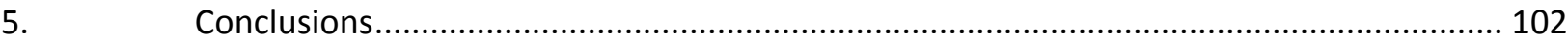

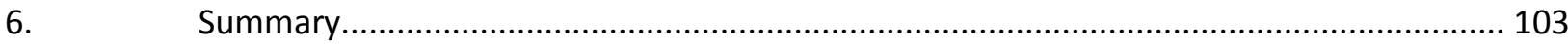

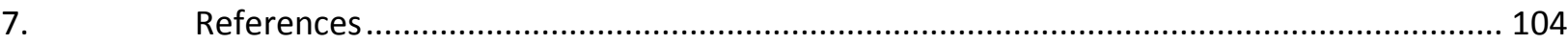

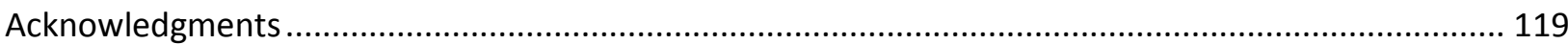

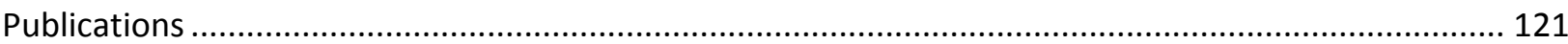

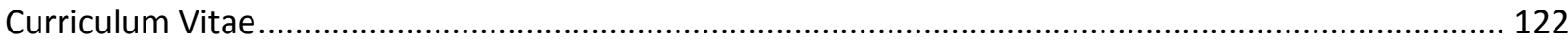




\section{List of the figures}

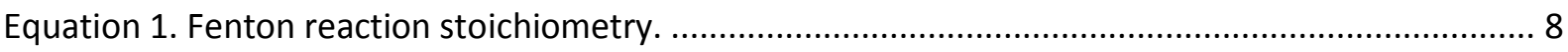

Figure 1.1. Scheme of the metal binding sites to aSyn ................................................................. 9

Figure 1.2. Scheme of the interaction between $\mathrm{X}$-ray and matter ................................................ 14

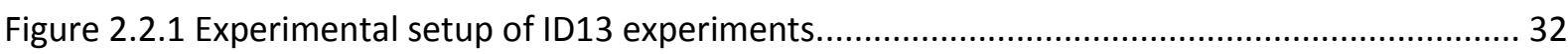

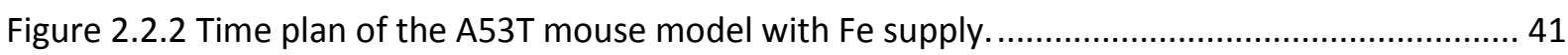

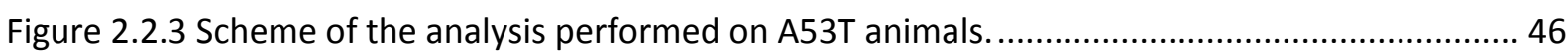

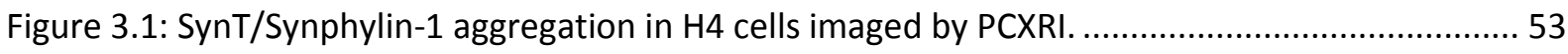

Figure 3.2 . WAXS analysis of PD sample and CTR sample after thresholding..................................... 54

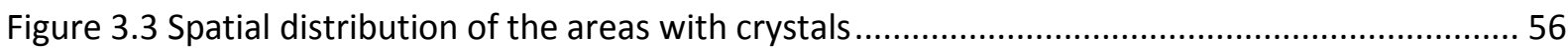

Figure 3.4 Histological evaluation of amyloid structure using Bielschowski silver stain. ..................... 57

Figure 3.5 A. Overlay of the four fluorescence spectrum calculated.............................................. 59

Figure 3.6 Relative elemental quantification of X-ray fluorescence from PD samples compared

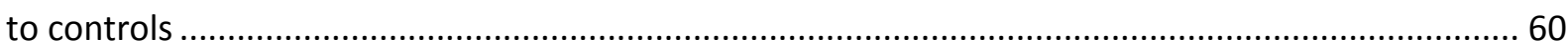

Figure 3.7 Example of total fluorescence maps and the maps for $\mathrm{Zn}$, Fe and $\mathrm{Cu}$.............................. 61

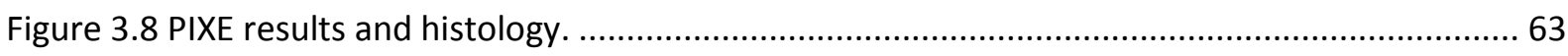

Figure 3.9 PIXE measurements of primary midbrain neurons overexpressing aSyn with or

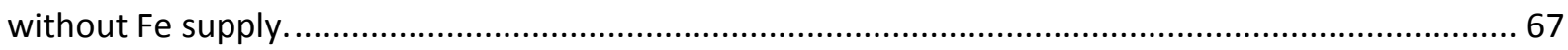

Figure 3.10 Evaluations of metal transport proteins levels influenced by increasing

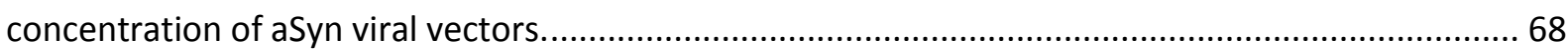

Figure 3.11. Evaluations of metal transport proteins levels influenced by increasing

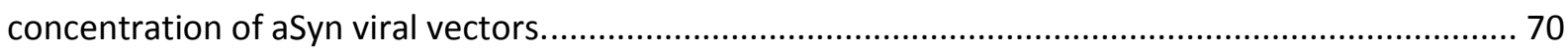

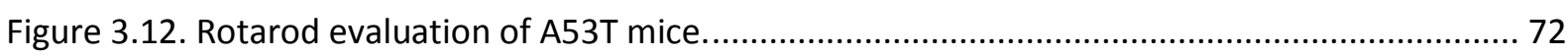

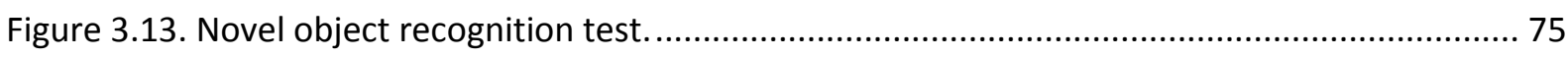

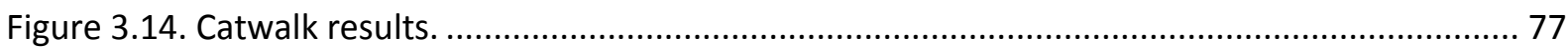

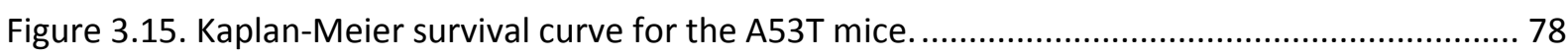

Figure 3.16 Dot blot assay for aggregated human aSyn in the cortex of A53T mice........................... 80 


\section{Abbreviation List}

6-OHDA: 6-hydroxydopamine

AAV: adeno-associated virus

$A \beta:$ amyloid $\beta$

AD: Alzheimer's disease

ALS: amyotrophic lateral sclerosis

APS: ammonium peroxide sulfate

ATP7a: Menkes' protein or copper-transporting P-type ATPase type a

ATP7b: Wilson disease protein or copper-transporting P-type ATPase type b

aSyn: alpha-synuclien

BSA: bovine serum albumin

Ca: calcium

$\mathrm{CaCl}_{2}$ : calcium chloride

Cl: chlorine

CNS: central nervous system

$\mathrm{CO}_{2}$ : carbon dioxide

CSF: cerebrospinal fluid

CTR1: High affinity copper uptake protein 1

Cu: Copper

Cy: cyanine

DA: dopamine

DAPI: 4',6-diamidino-2-phenylindole

DAT: dopamine transporter

DIV: days in vitro 
DMT1: divalent metal transporter 1 , or natural resistance-associated macrophage protein 2

DNA: deoxyribonucleic acid

ECL: enhanced chemiluminescence

EtOH: ethanol

EDTA: ethylenediaminetetraacetic acid

EGFP: enhanced green fluorescent protein

fc: fold change

FCS: fetal calf serum

Fe: iron

Fpn1: Ferroportin 1

GAPDH: Glyceraldehyde 3-phosphate dehydrogenase

GPe: external globus pallidus

GPi: internal globus pallidus

HBSS: Hank's Balanced Salt Solution $\mathrm{H}_{3} \mathrm{BO}_{3}$ : boric acid

$\mathrm{HCl}$ : hydrochloric acid

$\mathrm{H}_{2} \mathrm{C}_{6} \mathrm{O}$ : ethanol

$\mathrm{HNO}_{3}$ : nitric acid

$\mathrm{H}_{2} \mathrm{O}$ : water

$\mathrm{H}_{2} \mathrm{O}_{2}$ : hydrogen peroxide

HRP: horseradish peroxidase

ICC: immunocytochemistry

IHC: immunohistochemistry

$\mathrm{K}$ : potassium

LB: Lewy body

MAOB: monoamine oxidase $B$ 
MPP+: 1-methyl-4-phenylpyridinium ion

MPTP: 1-methyl-4-phenyl-1,2,3,6-tetrahydropyridine

mRNA: messenger RNA

$\mathrm{NaCl}$ : sodium chloride

$\mathrm{NaHCO}_{3}$ : sodium hydrogen carbonate

$\mathrm{Na}_{2} \mathrm{HPO}_{4}$ : sodium hydrogen phosphate

$\mathrm{NaN}_{3}$ : sodium azide

$\mathrm{NaOH}$ : sodium hydroxide

NDD: neurodegenerative disorder

NGS: normal goat serum

$\mathrm{NH}_{4} \mathrm{Cl}$ : ammonium chloride

NOR: novel object recognition test

ON: over night

P: phosphorus

PBS: phosphate buffered saline

PCR: polymerase chain reaction

PD: Parkinson's disease

PFA: paraformaldehyde

PIXE: particle-induced X-ray emission

PLO: poly-L-ornithin

PMN: primary midbrain neurons

PNS: peripheral nervous system

qPCR: quantitative PCR

RNA: ribonucleic acid

ROS: reactive oxygen species

rpm: rounds per minute 
RT: room temperature

S: Sulfur

SDS: sodium dodecyl sulfate

SDS-page: SDS-polyacrylamide gel electrophoresis

SEM: standard error of the mean

$\mathrm{Si}_{3} \mathrm{~N}_{4}$ : silicon nitride

SNP: single nucleotide polymorphism

SNpc: substantia nigra pars compacta

SNpr/SNr: substantia nigra pars reticulate

SAXS: small angle scattering

STXM: scanning transmission X-ray microscopy

STN: subthalamic nucleus

TBS: Tris-buffered saline

TBS-T: TBS-Tween20

TEMED: tetramethylethylenediamine

TfR: transferrin receptor

TH: tyrosine hydroxylase

Tris: 2-Amino-2-hydroxymethyl-propane-1,3-diol

VA/VL: thalamus

VTA: ventral tegmental area

WAXS: wide angle scattering

Zn: Zinc 


\section{Introduction}

\subsection{Neurodegenerative diseases}

There are more than 600 disorders that can afflict the nervous system. Among those, the term "neurodegenerative diseases" refers to the progressive damage or degeneration of various types of nerve cells, which can occur in the central or in the peripheral nervous system, causing a reduced innervation of target structures. Neurodegenerative diseases cause progressive disability in affected persons through the loss of sensory, motor and/or higher cognitive functions.

This kind of diseases increases its incidence with age and growing life expectancy in industrialized countries (ONU 2015) results in a higher prevalence of neurodegenerative disorders in the population. Among the neurodegenerative diseases the most frequent are Alzheimer's disease (Mayeux 2006) and Parkinson's disease (Pringsheim et al. 2014).

The costs of neurodegenerative diseases are enormous due to the high number of persons affected. The costs do no account only for the costs of treatment, but also the lost productivity of patients and their caregivers, for whom looking after chronically disabled family members can represent an enormous source of emotional, practical, and financial burden (Wright Willis et al. 2010).

Neurodegenerative diseases share many pathophysiological characteristics like the increased oxidative stress in the cells, protein aggregation mechanisms, mitochondrial dysfunction, increase programmed cells death and deficits in the protein recycling mechanisms (Mayeux 2006; Pringsheim et al. 2014).

Despite intense years of research, the causes of neurodegenerative diseases are still not completely understood, and the therapies available are only symptomatic as they are not capable to hamper the progression of the disease. Moreover, the diagnosis and the evaluation of disease severity of many neurodegenerative diseases is still largely based on the clinical examination of the patients, as reliable biomarkers are not yet available (Chen-Plotkin 2014). 
For those reasons, the investigation of the etiopathology of neurodegenerative diseases and of possible therapeutic strategies plays a role of primary importance for their future applications.

\subsection{Parkinson's disease}

Parkinson's' disease (PD) is the second most common neurodegenerative disease after Alzheimer's disease. The incidence (given per 100,000) is increasing with age: 41 in individuals 40 to 49 years; 107 in individuals 50 to 59 years; 173 in individuals 55 to 64 years; 428 in individuals 60 to 69 years; 425 in individuals 65 to 74 years; 1,087 in individuals 70 to 79 years; and 1,903 in individuals over age 80 (Pringsheim et al. 2014). Sex has an effect on the prevalence as males are significantly more affected than females (Pringsheim et al. 2014).

Characteristic symptoms of PD comprise core motor clinical features including rigidity, postural instability, resting tremor and bradykinesia. These are the symptoms that were described for the first time by a British physician named James Parkinson in 1817 in an essay called "An Essay on the Shaking Palsy" (Parkinson 1817). We owe the modern name to the French physician Jean-Martin Charcot (who described another disease, the amyotrophic lateral sclerosis for the first time), that changed the name from "shaking palsy" to Parkinson's disease.

One of the hallmark of PD is the presence of proteinaceous aggregates in the patients' brains. These aggregates take the name of "Lewy Bodies" (LB) after the German physician who discovered them in 1912: Frederic Lewy. However it was only in 1997 when an Italian biologist, Maria Grazia Spillantini, could identify the most abundant protein contained in LB: alpha-synuclein (aSyn) (Spillantini et al. 1997).

PD is mostly an idiopathic disease, but about the $5 \%$ of the cases have a genetic origin (Klein \& Westenberger 2012). Mutations on six genes are unequivocally linked to heritable PD: SNCA (the gene for aSyn), LRRK2 (PARK8), Parkin (PARK2), PINK1 (PARK6), DJ-1 (PARK7), and ATP13A2 (PARK9) (Klein \& Westenberger 2012). Many mechanisms involved in the pathophysiological processes of PD patients with familiar forms of the disease resemble very closely the features of the idiopathic patients. In 
fact, they show increased oxidative stress, mitochondrial dysfunction and protein misfolding (Dexter \& Jenner 2013).

The current diagnosis of PD occurs in late stage of the pathophysiological processes, as it is still strongly relying on the severity of patients' symptoms as specified in the Unified Parkinson's Disease Rating Scale (UPDRS)(Oung et al. 2015). At this stage about as much as $70 \%$ of dopaminergic neurons might be already degenerated (Oung et al. 2015).

Besides the aforementioned motor symptoms, PD is also linked to some other non-motor symptoms that consist of fatigue, nervousness, constipation, visual hallucinations, olfactory dysfunction, pain, urinary irregularities or urgency, extreme sweating, and sleep deprivation (Oung et al. 2015). Those non-motor symptoms are now emerging as early symptoms or pre-parkinsonian states. (Iranzo 2013; Oung et al. 2015).

\subsubsection{Pathophysiology of Parkinson's disease}

One of the most prominent histological features in PD is considered to be the damage to specific areas of the $\mathrm{SN}$, in the pars compacta (SNpc), with severe loss of dopaminergic cells and their projections, causing the appearance of the classical motor symptoms in patients (Galvan \& Wichmann 2008).

$\mathrm{SNPc}$ is a midbrain nucleus and is involved in the regulation of voluntary movement. In the physiological situation, the dopaminergic neurons are neuromelamin (NM) positive cells of the SNpc that have long and unmyelinated axonal projections into the striatum. The connections between the striatum and the basal ganglia are organized into two distinct pathways: the direct and indirect pathway. The direct pathway arises from a set of neurons that projects to neurons of the internal globus pallidus (GPi) and subtantia nigra pars reticulata (SNpr) and express preferentially the dopamine receptor D1, whereas the indirect pathway arises from a different set of neurons that projects to the external Globus Pallidus (GPe) and expresses preferentially the dopamine receptor D2 (Galvan \& Wichmann 2008). 
Dopamine therefore appears to play a double role as it can modulate the activity of the basal ganglia output neurons in GPi and SNpr by facilitation of transmission over the direct pathway, and inhibition of transmission over the indirect pathway. When the GPi nucleus is inhibited by the GABAergic cells activated but the direct pathway, the result is the excitation of the thalamus and of the motor cortex. On the other hand, the indirect pathway mediates the inhibition of the GPe that decreases the excitatory outputs of the subthalamic nucleus that projects to GPi. GPi itself has projections to the ventral nucleus of the thalamus and the ventral nucleus of the thalamus that projects to the motor cortex. The motor cortex controls behavior and gives input to the spinal cord, the striatum and the SNpr (Kandel et al. 2012).

This complex feedback system plays a pivotal role for the initiation of voluntary movement and it becomes impaired during the development of PD as the dopaminergic neurons contained in the SNpc are progressively degenerating thus causing the appearance of the motor symptoms.

When the clinical symptoms arise, about the $70 \%$ of dopaminergic neurons are already depleted (Galvan \& Wichmann 2008). It has been proposed that the degeneration starts at the synaptic terminals in the striatum and afterwards it propagates to the neurons in the SN causing progressive cell death (Burke \& O'Malley 2013).

\subsection{Alpha - synuclein in PD}

Together with the loss of dopaminergic neurons, the most important hallmark of PD is the presence of inclusion bodies. These inclusion bodies can be found mostly in the NM positive cell perikarya as globular structures where they are called Lewy bodies (LB); or they can be spindle-shaped in neurites: the Lewy neurites (LN)(Braak et al., 2003).

The development of PD and its severity seem to be regulated by the spreading of LB within different areas of the brain. Indeed, according to Braak staging, PD can be in its initial phases asymptomatic, but at this stage LB might be already present in the olfactory bulb, medulla oblongata and pontine tegmentum. In later stages the LB 
pathology extends to SN, the midbrain and basal forebrain. In the last stage LBs reach the neocortex leading to dementia (Braak et al., 2003).

The main component of LB has been found to be a protein called alphasynuclein (aSyn) (Spillantini et al. 1997). After this discovery, other diseases have been proven to be associated with aSyn aggregation, like multiple system atrophy, and dementia with Lewy Bodies. These diseases are collectively called "synucleinopathies" and also PD belongs to this group (Tagliafierro \& Chiba-Falek 2016).

In the etiopathology of PD, the role played by aSyn is very prominent, as some families with the hereditary form of the disease bear mutations in the gene for aSyn (SNCA). Several single point mutations of aSyn have been identified in those families: A53T, A30P, E46K, H50Q and G51D. Furthermore, duplications and triplications of the gene have been linked to inherited forms of the disorder (Kasten \& Klein 2013).

The physiological function of aSyn is not yet fully understood, nonetheless aSyn seems to facilitate the formation of the soluble NSF attachment protein receptor (SNARE) complex and it plays a role in dopamine (DA) biosynthesis and regulation (Burré et al. 2010). Interestingly, aSyn was shown to act as a cellular ferrireductase using $\mathrm{Cu}$ and $\mathrm{NADH}$ as co-factors to reduce Fe(III) to Fe(II) thus increasing the levels of oxidative stress in the cell (Davies et al. 2011).

\subsubsection{Alpha-synuclein aggregation}

aSyn is a small molecule of 140 aminoacids that can be found ubiquitously throughout the CNS and also in red blood cells (Barbour et al. 2008). Although it interacts with lipid membranes, it's a highly soluble protein. It's localized at the presynaptic terminals in close proximity to synaptic vesicles and in the cytosol. Under physiological conditions it is thought to be poorly structured therefore it can undergo several structural modifications including self-aggregation and fibril formation (Breydo \& Uversky 2011).

Several points of evidence indicate that aSyn plays a primary role in the pathophysiology of PD, in particularly, its misfolding appears like a crucial event in 
the disease development. Misfolded aSyn is the main component of the LB found in PD patients thus further supporting the importance of the protein in PD (Spillantini et al. 1997). Whether the aggregated forms of aSyn present in LB are per se toxic, is still under debate, but there is a large consensus that aggregated forms of aSyn, like oligomers, are able to exert cellular toxicity in in-vitro and in-vivo conditions (Berrocal et al. 2014). Moreover, even the mutant forms of aSyn that are responsible for familiar PD appear to have a higher propensity for aggregation and fibrillation, compared to WT aSyn leading to a premature onset of the disease (Berrocal et al. 2014).

aSyn sequence can be structurally divided into 3 main parts: a $\mathrm{N}$-terminal part (residues: 1-60), which interacts with membranes; a central NAC domain (non-Abeta component of Alzheimer's disease amyloid), that is a random coiled coil structure that can cause the misfolding into $\beta$-sheets (residues 61-95) and a C-terminal part (residues 96-140), which seems to hinder the fibril formation (Breydo \& Uversky 2011).

The exact mechanisms of fibril formation are still unclear. One of the most accredited proposed models, is that aSyn shifts its folding from monomer to fibrils enriched in $\beta$-sheets, through several different intermediate steps. Some of the most common aggregated forms of aSyn are oligomers, pre-fibrils, annular and granular structures. They constitute a roughed energy funnel of the different conformations of the protein that can lead to a proper folding in physiological conditions, or to the misfolding and to the pathology.

The various intermediate steps are differently folded and are sometime called "strains", a loaned term taken from the prion research. Together with the peculiarity of the folding, the different strains of aSyn appear to exert also different levels of toxicity through different mechanisms in in vivo and in vitro models (Peelaerts et al. 2015).

Recent findings point out that the conversion of aSyn into its misfolded species is also a crucial step in the spreading of aSyn throughout the brain for the progression of the PD pathology. This resembles a prion-like mechanism that could explain the different stages of the disease proposed by Braak (Recasens \& Dehay 2014). 


\subsubsection{Alpha-synuclein and metals}

Metals seem to play a pivotal role in the etiopathology of PD. In fact, one of the most interesting features of PD patients, besides aSyn misfolding, is the metal dyshomeostasis. PD patients' brains display an increased amount of Fe in the SN (Dexter et al. 1989). Moreover, there is a co-localization of Fe and LB at the histological level (Castellani et al. 2000).

Adequate amounts of trace metals are needed in the normal physiology of the brain. Due to their red-ox potential, metals provide counter-ions and they constitute the catalytic core of enzymes as electron carriers and/or red-ox reactions. Nonetheless, metals can exert also toxic effects in the organism by increasing the levels of reactive oxygen species (ROS) when the red-ox potential isn't counterbalanced. This process is described by the Fenton reaction (see Equation 1):

$$
\begin{aligned}
& \mathrm{M}^{\mathrm{n}+1}+\mathrm{O}_{2}^{-\cdot} \rightarrow \mathrm{M}^{\mathrm{n}}+\mathrm{O}_{2} \\
& \mathrm{M}^{\mathrm{n}}+\mathrm{H}_{2} \mathrm{O}_{2} \rightarrow \mathrm{M}^{\mathrm{n}+1}+\mathrm{OH}^{-}+\mathrm{OH}^{\bullet} \\
& \text { Net reaction }(1)+(2) \text { : } \\
& \mathrm{O}_{2}^{-\bullet}+\mathrm{H}_{2} \mathrm{O}_{2} \rightarrow \mathrm{O}_{2}+\mathrm{OH}^{-}+\mathrm{OH}^{\bullet}
\end{aligned}
$$$$
\text { Haber-Weiss reaction (3) }
$$

Fenton reaction (2)

Equation 1. Fenton reaction stoichiometry.

The result given by the Fenton reaction is the production of the highly reactive hydroxyl radicals that are able to oxidize proteins, DNA and lipids thus leading to cytotoxicity and metabolic impairment.

The ROS production is usually hindered by several detoxification mechanisms like chaperone proteins and anti-oxidant molecules (like ascorbic acid). These systems appear to be impaired in neurodegenerative diseases in general and in PD in particular (Dias et al. 2014).

Several metals can directly bind to aSyn fostering its aggregation process (Uversky et al. 2001). A scheme of aSyn structure and the binding sites for metals is depicted in Figure 1.1 from (Carboni \& Lingor 2015). The affinities of aSyn for metals 
are unequal. $\mathrm{Cu}(\mathrm{II})$ binds at the $\mathrm{N}$-terminus of the protein with the highest affinity among the metals as the dissociation constant is of approximately $0.1 \mu \mathrm{M}$ when the binding occurs at the first 35 aminoacid residues of the protein (Binolfi et al. 2006). aSyn has another binding site at the C-terminus that is a so called "dirty site" as it can bind several divalent metals without a great selectivity. In fact, the dissociation constant for $\mathrm{Fe}(\mathrm{II}), \mathrm{Mn}(\mathrm{II})$ and $\mathrm{Cu}(\mathrm{II})$ binding at the $\mathrm{C}$-terminus is in the range of $1 \mathrm{mM}$ and it's similar for the three metals (Binolfi et al. 2006).

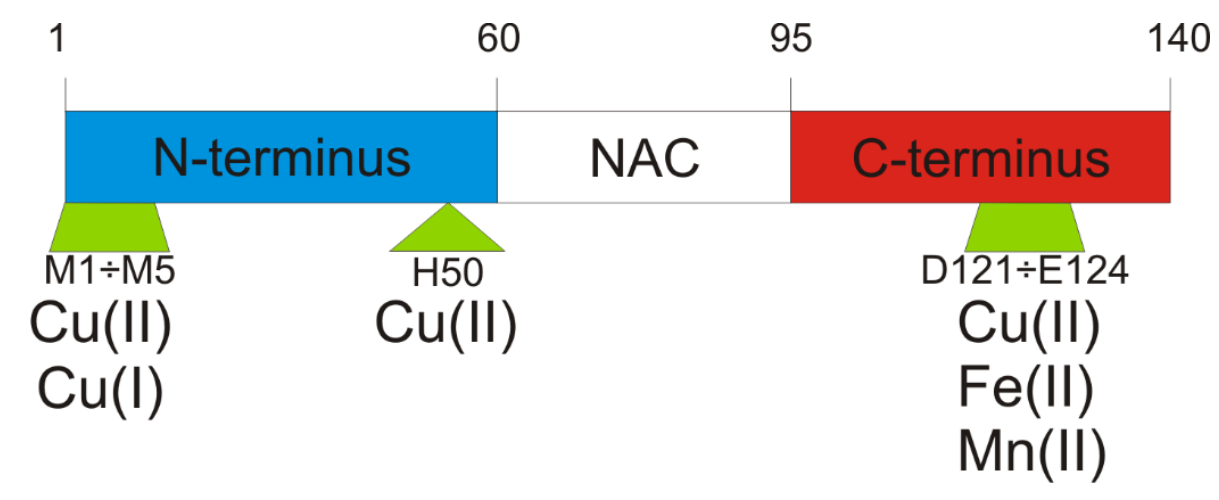

Figure 1.1. Scheme of the metal binding sites to aSyn. Modified from (Carboni \& Lingor 2015)

\subsubsection{Animal models of Parkinson's disease}

As already mentioned in the previous paragraphs, the etiopathology of PD is still not completely understood and the available therapies can only alleviate patients' symptoms but are not curative. To this end, further studies are needed and the use of animal models is a precious tool because it can help a deeper understanding of disease progression and explore possible treatments.

At the moment there is no currently available model that can reproduce all the pathological features of PD (Jagmag et al. 2016). Nonetheless, the animal models applied in research so far have contributed extensively to deepen the knowledge regarding the mechanisms underlying PD pathology. The models used in research can be categorized in two main groups. First, there are pharmacological models where a toxin exerts its effects on dopaminergic pathway. Second, there are genetic models where there transgenic animals express proteins linked to the PD pathology. 
Pharmacological models: the pharmacological models that are employed for modeling PD rely on the effect of neurotoxins that cause nigrostrial DA neuronal degeneration. He most widely used are:

- 6-OHDA: the 6-hydroxydopamine (6-OHDA) was the first toxin used in rats and in mice to cause nigrostriatal degeneration. This drug has to be injected directly in the brain in the SNpc, Medial Forebrain Bundle (MFB) or striatum as it cannot cross the blood brain barrier (BBB). Its main action is the increase of the production of reactive oxygen species and the consequent death of DA neurons as well noradrenergic nerve terminals (Metz et al. 2005). Lesioned animals display motor impairments in the contralateral side of the body to the injection side, and are responsive to the treatment with levodopa but fail to show presence of intracytoplasmatic inclusions.

- MPTP: 1-methyl-4-phenyl-1,2,3,6-tetrahydropyridine (MPTP) is the prodrug to the neurotoxin MPP+. MPP+ is capable of destroying DA neurons in the $\mathrm{SN}$ and in the striatum of the brain. It has been historically discovered in drug users that displayed parkinsonian-like syndrome as MTPT was a byproduct of the chemical synthesis of some illicit drugs (Langston et al. 1984). Unlike the 6-OHDA, MPTP can cross the BBB and it is converted into $\mathrm{MPP}+$ by the monoamine oxidase B of the glial cells. Once MPP+ enters the DA neurons through the membrane dopamine transporters, it exerts its toxic function by inhibiting the complex I of the respiratory chain in the mitochondria (Jackson-Lewis \& Przedborski 2007). It is widely used as model in mice, and it is usually applied as acute treatment paradigm. Behavioral features of the model consist in the reduced movement of the animals. Intriguingly, chronic treatment in mice lead to the appearance of inclusions in the brain that are reactive for aSyn and ubiquitin (Fornai et al. 2005).

- PESTICIDES: Rotenone and Paraquat are two pesticides that have been used as toxins to mimic PD features in mice. Rotenone toxicity mechanisms are similar to MPTP causing the nigrostriatal DA degeneration, but its use is limited by its high toxicity and the high mortality of the animals after treatment (Moretto \& Colosio 2011). Paraquat is an herbicide that has 
similar effect to Rotenone, but is used to a lesser extent due to the high variability of the outcomes (Moretto \& Colosio 2011).

Genetic models: the genetic animal models of PD are taking into account five genes that have been known to have causal connection in familial PD: aSyn; LRRK-2; Parkin; DJ-1 and PINK-1 (Jagmag et al. 2016). Even if PD isn't due to a mutation on a single gene locus but it's rather a multifactorial disease, the genetic models enable to understand the signaling and pathways associated with known causal gene, and how the perturbation of the signaling is effecting disease-like phenotypes in the animals.

- Alpha-synuclein models: aSyn TG animal models have been extensively studied due to the growing body of literature demonstrating a tight link between PD and this protein. Research has focused mainly on the knock down of the protein or the knock in of several variants of human aSyn (wildtype, A30P, A53T or A30P+A53T) through a transgenic approach with various promoters or by viral vectors, and combined with chemical perturbations (Deng \& Yuan 2014). aSyn knockout mice were mildly motor impaired since they showed reduced rearing activity in the open field. From a biochemical point of view, the animals displayed decreased DA content in the striatum, and a decrease of the reserve pool of vesicles in the hippocampus (Fleming et al. 2005).

Among all those models, a special remark in this section should go to the model employed for this thesis: the TG strain called "B6;C3-Tg(PrnpSNCA*A53T)83Vle/J" (addressed only as A53T mice from now on). This model was reported for the first time by Giasson and colleagues in 2002 (Giasson et al. 2002).

The A53T mice express the single point mutation of human aSyn, responsible for a familiar form of PD (Polymeropoulos 1997) under the prion protein promoter. This mouse line displays a progressive motor phenotype from the $7^{\text {th }}$ month of age, starting with neglect of grooming, weight loss, and reduced ambulation. After that, a progressive paralysis of the limbs begins at the hind legs and it extends within days also to the front limbs (Giasson et al. 2002). Moreover, A53T animals have proven to fail 
motoric tests like Catwalk (Tatenhorst et al. 2016) and behavioral tests like the radial maze (Graham \& Sidhu 2010) and displayed less anxiety compared to the WT (Graham \& Sidhu 2010).

The histology of the A53T animals depicts the progressive development of aggregated forms of human aSyn in cells perikarya in the CNS, especially in the cerebellum, spinal cord, locus coeruleus and raphe nuclei. These inclusions can be found also in dystrophic neurites, reminiscing LN (Giasson et al. 2002). Intriguingly, the inclusions are triton-X 100 resistant and resistant to proteinase $K$ digestion (Tsika et al. 2010; Tatenhorst et al. 2016). As the mice age, the phenotype of the model is more marked, because it's progressive and it resembles in many features the development of familiar forms of PD. In fact, there is no aSyn pathology in the young animals and the onset of the behavioral deficits coincides with the appearance of the aSyn inclusions (Giasson et al. 2002).

\subsubsection{Use of chelators in neurodegeneration and Parkinson's disease}

The traditional therapies for PD are still commonly used and they are actually quite effective in the first phases of the disease to alleviate the symptoms. Unfortunately, these therapies are not able to stop the progression of the disease. Among the most widely used treatments there are DA precursors (e.g. levodopa), DA agonists, inhibitors of DA uptake (MAOB inhibitors), and anti-glutamatergic or anticholinergic molecules (Connolly \& Lang 2014).

However, due to the increasing interest on the interaction between aSyn and the metals, also new complementary therapeutic strategies such as chelator therapies are being exploited. Chelators are a class of pharmacological compounds that exhibit a high affinity for metals. These small molecules can sequester metals hampering their toxic effects and reducing the ROS production (Perez et al. 2008).

Research carried out so far (in vitro and in vivo) employ the use of Fe chelators, above other metals, as the Fe accumulation in PD patients is well documented (Dexter et al. 1989). 
Desferrioxamine, for instance, has been proven to protect up to $60 \%$ of the DA neurons from death in mice injected with 6-OHDA thus eliciting positive effects in this model. It could also hinder the ROS production. This chelator, however, fails to cross the BBB (Youdim et al. 2004).

Another promising chelator drug used in that kind of studies is deferiprone (DFP). DFP is a bidentate chelator for Fe(II) that can cross the BBB that is currently use as treatment for the Fe overload in thalassemia major (Perez et al. 2008).

Preclinical studies of PD using 6-OHDA models pointed out a better survival of TH positive neurons in the SNpc and higher DA levels in the striatum by administering $10 \mathrm{mg} / \mathrm{kg}$ bw of DFP twice daily for 5 days. Therefore the treatment with DFP appears to have beneficial effects in this murine model (Workman et al. 2015).

Regarding clinical trials, a small pilot study with about 40 patients was carried out with DFP (Devos et al. 2014) and at the moment three other trial studies on PD are being conducted with the same drug (Clinicaltrials.gov - Bethesda (MD): National Library of Medicine (US). [cited 2016 Aug]).

The PD patients in the Devos study received $30 \mathrm{mg} / \mathrm{kg} /$ day of DFP in association with the classical DA agonist treatments. This study points out that DPF has a beneficial effect in the patients that took the drug at early stages of the disease (within 3 years from the diagnosis). As long as they took DFP, the UDPRS score was kept stable in comparison to patient who tool placebo and the Fe accumulation in the brain was proved to be reduced in the MRI (Devos et al. 2014). The follow up of the study pointed out that PD patients with a reduced ceruplasmin-ferroxidase activity could respond better to the DFP treatment (Grolez et al. 2015).

\subsection{X- ray imaging techniques and their principles}

X-rays were discovered in 1895 by a German physicist named Wilhelm Conrad Röntgen, who named them as we know it today X-rays, because their nature was, at that time, unknown. For this discovery he was awarded the Nobel Prize 1901.

$\mathrm{X}$-rays are part of the electromagnetic radiation spectrum. Their wavelength is usually between $10^{-8} \mathrm{~m}$ to about $10^{-11} \mathrm{~m}$. X-rays can be "hard X-rays" when their 
energy is above $5 \mathrm{keV}$ with a wavelength below $10^{-10} \mathrm{~m}$; while at lower energies and at higher wavelength they are called "soft X-rays".

Due to their wavelength, $\mathrm{X}$-ray photons can interact with matter causing three main different phenomena (see Figure 1.2): photoelectric absorption, coherent scattering (Thomson/Rayleigh scattering) and incoherent scattering (Compton scattering).

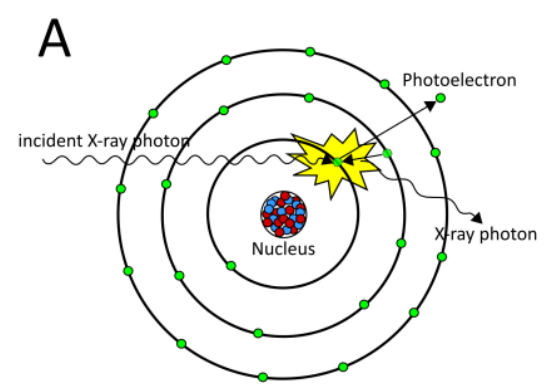

Photoelectric absorption

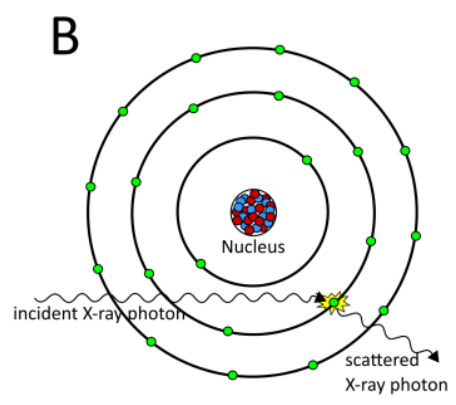

Rayleigh scattering

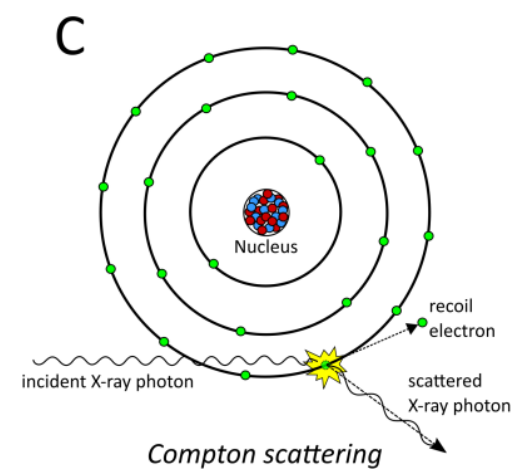

Compton scattering

Figure 1.2. Scheme of the interaction between X-ray and matter. A. photoelectric absorption; B. Coherent (Rayleigh) scattering; C. Incoherent (Compton) scattering. Modified from (Seibert \& Boone 2005).

The photoelectric absorption process is a phenomenon that occurs when an incident photon is interacting with an electron in the orbital position in the $\mathrm{K}, \mathrm{L}$, or $\mathrm{M}$ shell of the scattering atom. The electron absorbs the photon energy and a photoelectron is ejected from the orbital. The orbital position vacated by the photoelectron is filled by an electron from an outer orbital. The variation of energy of the electron that goes form a higher energy level to a lower energy level is resulting in the emission of a photon (Alpen 1998). X-ray fluorescence relies on the photoelectric absorption as its principle.

The coherent scattering (Thomson scattering) is the phenomenon that occurs when no energy of the incident photon is converted into kinetic energy in the medium. In this kind of interaction, the energy and frequency of the incoming photons with the electrons cause the oscillation of the electrons of the medium at the same energy and frequency. The electrons of the medium therefore emit an electromagnetic radiation of the same frequency of the incident photon. The emitted electromagnetic radiations from each of the electrons combine and/or interfere with each other causing an emission of radiation with direction different from that of the 
incoming photon but with the same energy. The direction of the radiation is depending on periodical geometry of the atoms in the medium (Alpen 1998). X-ray diffraction techniques rely on the coherent scattering effects.

The incoherent scattering (Compton scattering) is the phenomenon that occurs when the incoming photon and the scattered electromagnetic wave coming from the interaction with the medium have different wavelengths. The process involves the interaction of a photon and an electron that is weakly bound, but the original photon reappears as a scattered photon of lower energy. On average, the fraction of energy transferred to kinetic energy per interaction increases with photon energy (Alpen 1998).

There are two main kinds of X-ray sources: laboratory sources (like X-ray tubes and X-ray lasers) and synchrotron facilities. The latter provide usually the best sources for imaging and spectroscopy because of their high brilliance (highly collimated photon beam generated by a small divergence and small size source). Moreover, synchrotrons provide a very broad spectrum of electromagnetic radiation.

Synchrotron radiation is created by deflecting the trajectory of an electron or positron beam traveling at relativistic speeds. The movement of the particles is induced by bending the electron beam by the means of electromagnets or insertion device systems like undulators or wigglers. Electrons are forced to make many wiggles instead of a single bend by undulators or wigglers that are usually present in the straight sections of the storage ring. This leads to a multiplication of the emitted X-ray intensity. As a result, the synchrotron radiation possess high brightness, a partial coherence and polarization (Gianoncelli et al. 2015).

\subsubsection{X-ray fluorescence and particle induced X-ray emission: principles and uses}

X-ray Fluorescence (XRF) and Particle-Induced X-ray Emission (PIXE) are two analytical techniques that allow the simultaneous detection of several elements of the periodic table in a nondestructive way. The basic principles are described here according to (Verma 2007). 
Both techniques allow the simultaneous measure of the elemental content in a qualitative (by wavelength) and quantitative (by intensity) manner. They can be used as raster scanning techniques, thus providing also spatial information about elemental distribution in the sample. Therefore these techniques are applied to a variety of different fields like biology, geology and material sciences.

The basic principle is similar for both techniques, as they rely on the ejection of an electron from an inner shell of an atom while another electron from a higher shell shifts into this lower shell to fill the energy gap left behind. The energy lost from the electron that goes from a higher energy shell onto a lower energy shell results in the emission of a photon with an energy that equals the energy difference between the two shells.

The main difference between PIXE and X-ray fluorescence is the mechanism by which the inner-shell electron is ejected. In fact, in XRF there is high energy X-ray photon beam that enables the ejection of the electrons from the inner-shell; on the contrary PIXE takes advantage of a beam of charged particles (like He-ions or protons) to produce the electron ejection.

The laws of Quantum Mechanics predict how much energy must be lost by the electron in order to occupy the inner-shell state. Each electron transfer, for example from the L-shell to the K-shell, represents a loss in the potential energy of the atom and it is specific for each given element. The energy lost between an L-shell to the Kshell is a photon called K $\alpha$ photon. The K $\alpha$ photon possesses an energy that can be calculated as the difference between the binding energies of the filled outer shell and the vacant inner-shell. The fraction of radiative (X-ray) decays is called the fluorescence yield, and is high for deep inner-shells.

The detection of the emitted photon in XRF and PIXE occurs normally in the regime of energy dispersion. The detection is usually done with $\mathrm{Si}(\mathrm{Li})$ detectors. The principle of these kinds of detectors is that the absorption of the characteristic photons in the intrinsic zone of the Si crystal. This causes a number of electron-hole pairs directly proportional to the absorbed energy that form a charge pulse, collected at the respective electrodes on the contact layers across the surface (Wobrauschek et al. 2010; Rodriguez-Fernandez et al. 1994). 
Since PIXE utilizes a beam of charged particles, it can be coupled with the Rutherford backscattering spectrometry (RBS). This kind of spectrometry takes advantage of the elastic backscattering of incoming ions that do not possess energies high enough to penetrate the Coulomb barrier. This technique is very useful in order to determine the mass of the scattering atomic nuclei present in the sample, because their depth can be calculated form the energy loss of the backscattered ion as it passes into and out of the sample.

The information given by RBS is used in combination with PIXE, as it can give insights of the matrix determination and depth of the sample, while PIXE itself gives the elemental profiling. The RBS/PIXE combination can be used successfully in the measurement of biological samples as the matrix is mostly composed by light elements such as carbon, nitrogen and oxygen that are easily resolved by RBS and the PIXE can effectively measure the concentration of trace metals. The results of this combination is a determination of the trace metals as absolute concentrations as the RBS allows the correction for the sample thickness of the events measured by PIXE (Johansson et al. 1995).

\subsection{2. $\quad \mathrm{X}$ ray scattering- based imaging techniques: SAXS, WAXS and STXM}

$X$-ray diffraction (XRD) is a non-destructive technique that gives information on crystals and highly ordered structures. XRD can determine the orientation of a single crystal or grain, measure the average spacing between layers or rows of atoms, determine the orientation of a single crystal and also find the crystal structure of an unknown material (Seeck \& Murphy 2015).

The principle of XRD is the effect produced by the coherent scattering of a beam of incident photons through a highly ordered material (usually a crystal) that produces sharp diffraction peaks. Since the interaction taken into account in XRD are coming from elastic scattering phenomena, the diffraction patterns produced by crystals can be described with a good approximation by classical physics using the Bragg's law of diffraction. The scattering patterns are usually recorded on a 2D 
detector and they can be used to extrapolate information in the crystal lattice geometries (Seeck \& Murphy 2015).

Small and wide angle diffraction: Small-angle X-ray scattering (SAXS) and wideangle X-ray scattering (WAXS) are two subtypes of XRD and work with the same principle. The SAXS setup records monochromatic scattering with a diffraction of few degrees, while WAXS records wider scattering angles. SAXS and WAXS can be often recorded in the same setup by moving the detector closer or further away from to the sample. Their readout gives the electronic density of a sample matrix. Higher dispersions result in higher scattering.

Crystalline materials are highly ordered structures that are conventionally analyzed in the WAXS range as it gives information on the interplanar distance of the atoms, while SAXS is more suited for amorphous crystalline structures (Seeck \& Murphy 2015). For this reason, SAXS has been applied in monodisperse solutions and it's capable of providing low resolution structures of the protein when it's coupled to mathematical modelling approaches (Graewert \& Svergun 2013).

SAXS has also been applied directly on eukaryotic cells and bacteria. In the amoeba Dictyostelium discoideum it showed fiber bundles of the actomyosin network and their orientation (Priebe et al. 2014). More recently nano- and microfocused SAXS revealed a highly ordered and intense signal of human mesenchyma upon differentiation (Bernhardt et al. 2016).

Scanning transmission X-ray Microscopy: Scanning transmission X-ray Microscopy (STXM) employs a coherent beam that is used for raster scans of a probe and a detector. There are two systems for the signal acquisition: bright-field and darkfield. In the bright-field the image represents the absorption map of the specimen; the dark-field image is produced by putting a beamstop on the detector in order to select first diffraction order of scattered radiation. This diffraction signal results that strongly scattering features appear bright while open areas of the specimen produce no scattered signal and appear dark in the image (Morrison \& Browne 1992). STXM can be applied to a variety of samples including biological specimens. Indeed, when applied to epithelial cell lines, it could demonstrate the presence and orientation of keratin bundles (Weinhausen et al. 2012). 


\subsection{Aim of the thesis}

To date, despite intense years of research, there is still no comprehensive understanding of the mechanisms that underlie PD pathology, as it appears to be a multifaceted disease and its causes are still elusive. There is, however, a large consensus on the pivotal role played by aSyn, and particularly in its aggregation processes and its interactions with metals.

Biophysical techniques such as X-ray crystallography or NMR represent a powerful tool for studying processes that lead to the formation of aSyn aggregates. Traditionally, these techniques are mainly carried out in systems that are far from physiological conditions, such as highly purified proteins in crystalized structures or proteins in liquid milieu at high concentrations. Indeed, there are usually a plethora of aSyn interactors that are neglected in those experiments and the physiological concentrations of proteins in the cells are far lower from those needed for studies.

At the same level, conventional biology techniques like cell cultures or histology can tell us a lot of what happens in cells and tissue of PD models. Unfortunately, most of these methods lack of spatial resolution as they need to be carried out in tissues or cells homogenates. Furthermore, they rely heavily on the use of tracers and antibodies, that are prone to artifact production and they are unable to give us the structural insights that appear to be so crucial for understanding aSyn-caused pathology.

To this end, the aim of this thesis was trying to combine biophysical techniques together with biological techniques striving to gather better insights in the pathophysiological mechanisms of PD. We collected the structural information and analyzed the metal content on cells and tissues with biophysical techniques while performing several complementary biochemical and histological characterizations.

Since the topic is very wide and can be exploited in a variety of different ways, this thesis strived to answer four main questions. 


\section{Is it possible to visualize aSyn aggregates through $\mathrm{X}$-ray measurements? Can} we have insights on the pre-aggregates structures?

Structural characterization data for aSyn come mostly from NMR or crystallography but little is known about aSyn structure into cells and tissues. To answer this question, two systems were applied: a cellular model of aggregation analyzed by scanning transmission X-ray microscopy; and sections of tissue coming from PD patients with possible LB presence. The goal was to characterize the scattering signal of aSyn. Moreover we aimed to search for possible signal of preaggregated forms in order to have structural insights on aSyn and a better knowledge of the processes that bring aSyn to aggregation in tissues.

\section{Do metals have an impact on aSyn aggregation in PD patients' tissue?}

In vitro structural analysis of aSyn aggregation pointed out that metals presence facilitates aSyn aggregation in vitro. Whether this happens in the real course of PD disease is still unclear.

To that, we performed two sets of experiments on tissue coming from PD patients compared to controls.

The first experiment took advantage of X-ray fluorescence on PD patients' tissue sections. The goal was understanding whether there was a correlation between the signal coming with the X-ray diffraction and the metal distribution. $\mathrm{A}$ comparison between the structural signal and the elemental distribution could prove whether the in vitro findings are supported by similar data from PD patients' post mortem tissue.

The second experiment was devised to analyze, by the means of particle induced X-ray emission (PIXE), whether the metal dys-homeostasis in PD patients' brains is due to the LB presence or it is regardless of aggregation and is homogenous throughout the region. For each sample that was imaged with PIXE, adjacent slices were cut and searched for LB with histological techniques. The LB found in the NM positive cells were imaged with PIXE and compared with NM positive cells of PD patients without LB and with NM positive cells of Ctr. 


\section{Which effect has aSyn on the metal content of the cell? Can aSyn have an} impact on the metal handling proteins in the cell?

There is a large consensus in the literature about the metal dys-homeostasis in the brains of PD patients' especially in the SN. It is still unknown, however, whether aSyn has a direct effect on the dys-homeostasis maybe having an effect on the expression of the proteins responsible for metal trafficking.

To study this, primary midbrain neurons were transfected with viral vector to overexpress aSyn (or EGFP as Ctr) and the metal levels as well as their distribution were analyzed by PIXE. Moreover, since in PD there is an increased Fe concentration in the $\mathrm{SN}$ of the patients, the same model was exploited with the addition of Fe.

A similar experimental set up was used for assessing the levels of six of the most important metal-trafficking proteins in cells transfected with aSyn or Ctr viral vector. The metal transporters were analyzed both at the protein level as well as the transcriptional level to assess the possible effect of aSyn overexpression.

4. Is it possible to modulate aSyn aggregation in an in vivo model by providing Fe supply?

It is well documented that there is an interaction between aSyn and Fe at the in vitro level as seen in biophysical studies. However, whether there might be a direct interaction of aSyn in an in vivo model of aggregation with Fe had not been assessed.

To that, A53T TG animals were intoxicated with Fe and different behavioral tests were performed. Moreover, animals received a treatment with the Fe chelator DFP to contrast the influence of Fe, thus providing valuable information on the effects of a possible future treatment for PD. 


\section{Materials and methods}

\subsection{Materials}

\subsubsection{Chemicals and companies}

Applichem: acrylamide, bovine serum albumin (BSA), ethylenediaminetetraacetic acid (EDTA), ethanol absolute, glycine, isopropanol, nitric acid $\left(\mathrm{HNO}_{3}\right)$, methanol, non-fat dried milk, sodium hydroxide $(\mathrm{NaOH})$, oxygen peroxide $30 \%\left(\mathrm{H}_{2} \mathrm{O}_{2}\right)$, p-coumaric acid, RNase-ExitusPlus, sodium dodecyl-sulphate (SDS), sucrose, sudan black, Tween-20

Apotex Inc.: Deferiprone (DFP)

Biorad: Dual protein standard, IQ Multiplex Powermix

Biochrom: Fetal calf serum (FCS)

Calbiochem: luminol

Dako: antibody diluent

Gibco: B-27 supplement, Dulbecco's Modified Eagle Medium F-12 (DMEM F12), Hank's Balanced Salt Solution (HBSS), Opti-MEM

Jackson laboratories: Normal donkey serum (NDS), normal goat serum (NGS),

Lonza: glutamine, penicillin/streptomycin solution (pen/strep)

Merck: potassium chloride $(\mathrm{KCl})$, sodium chloride $(\mathrm{NaCl})$, sodium phosphate monoacid $\left(\mathrm{Na}_{2} \mathrm{HPO}_{4}\right)$, sodium carbonate monoacid $\left(\mathrm{NaHCO}_{3}\right)$,

Peqlab: DirectPCR Lysis Reagent Tail

Promega: FuGENE HD Transfection Reagent

Qiagen: QuantiTect SYBR Green PCR Kit, QuantiTect Reverse Transcription Kit, RNA-free water

Roth: Acetic acid, citric acid, eosin Y, glycerol, hemalaun solution, hydrochloric acid $(\mathrm{HCl})$, para-formaldehyde (PFA), phosphate buffer solution (ready to dissolve PBS), tetramethylethylenediamine (TEMED) 
Sigma: 1-bromo-3-chloropropane, ammonium persulfate (APS), 4',6-Diamidin2-phenylindol (DAPI), Dithiothreitol (DTT), DPX mountant medium, mineral oil, Mowiol 4-88, sodium azide $\left(\mathrm{NaN}_{3}\right)$, poly-d-lysine (PDL), TRI Reagent, Trizma base

ThermoFisher Scientific: bicinchoninic acid assay (BCA), trypsin

\subsubsection{Antibodies}

Primary antibodies:

Alpha-synuclein: BD \#610786 (pan aSyn Ab, for WB and ICC); Invitrogen \#32-8100 (anti hu-aSyn, for WB); Covance \#Sig-39726 (anti hu-aSyn, for IHC);

ATP7a: Bioss \# bs-1572R (for WB);

ATP7b: Bioss \# bs-1718R (for WB);

CTR1: Cell Signaling \#13086 (for WB);

DMT1: Alpha Diagnostic \#NRAMP24-A (for WB);

Fpn1: Alpha Diagnostic \#MTP11-S (for WB);

GAPDH: Hytest \#9GH5 (for WB);

TfR: Invitrogen: 13-6800 (for WB);

V5-tag: Abcam \#ab9116 (for WB and ICC);

Secondary antibodies:

Cy2 goat anti-mouse: Jackson \#115227003

Cy3 goat anti-rabbit: Dianova \#111-165-006

Goat anti-mouse HRP conjugated: Dianova \#115-035-166

Goat anti-rabbit HRP conjugated: Jackson \# 111-036-003 


\subsubsection{Equipment}

Bead mill homogenizer Precellys 24

Blades Teflon-coated DB80 LX

Cage incubator

Camera Legria HFM36

CatWalk XT

Cawomat 2000 IR

CFX96 Real time system C1000

Touch

Ceramin beads $1 \mathrm{~mm}$

Cohan-Vannas Spring Scissors -

$6 \mathrm{~mm}$

Cover slides $24 \times 60 \mathrm{~mm}$

Cryostat CM $3050 \mathrm{~S}$

Cryomatrix $3801480 \mathrm{~S}$

Cyclone 25 PCR thermocycler

Dumont \#5 Mirror Finish Forceps

Dumont Reverse Action Forceps

Electrophoresis power supply

Micro-centrifuge 5415R

Mini-PROTEAN Tetra-Cell system

NanoDrop 1000

Peristaltic perfusion pump

Plastic feeding tubes, 22ga $\times 25 \mathrm{~mm}$

Rotarod 47600

$\mathrm{Si}_{3} \mathrm{~N}_{4}$ membranes SiRN-12.5-200-

8.0-500

$\mathrm{Si}_{3} \mathrm{~N}_{4}$ membranes SiRN-5.0-200-

3.0-500

SuperFrost Plus Microscope Slides

Spark $10 \mathrm{M}$ microplate reader

Whatman Schleicher \& Schuell

Minifold I
Peqlab (Erlangen, Germany)

Leica Microsystems (Mannheim, Germany)

Okolab (Pozzuoli, Italy)

Canon (Krefeld, Germany)

Noldus (Wageningen, The Netherlands)

Cawo (Düsseldorf, Germany)

BIO-RAD (Munich, Germany)

Peqlab (Erlangen, Germany)

Fine Science tools (Heidelberg, Germany)

Menzel (Braunschweig, Germany)

Leica Microsystems (Mannheim, Germany)

Leica Microsystems (Mannheim, Germany)

Peqlab (Erlangen, Germany)

Fine Science tools (Heidelberg, Germany)

Fine Science tools (Heidelberg, Germany)

GE Healthcare (Chalfton St. Gilles, UK)

Eppendorf (Hamburg, Germany)

BIO-RAD (Munich, Germany)

Thermo Scientific (Waltham, CA, USA)

Idex (Wertheim, Germany)

Instech Laboratories (Plymouth Meeting,

PA, USA)

Ugo Basile (Gemonio, Italy)

Silson (Southam, UK)

Silson (Southam, UK)

Menzel (Braunschweig, Germany)

Tecan (Männedorf, Switzerland)

Sigma (Darmstadt, Germany)

\subsubsection{Buffers and solutions}

Blocking solution ICC: $0.1 \%$ TritonX 100 and $1.5 \%$ NGS (normal goat serum, in PBS)

Blocking and antibody solution for IHC: $2.5 \%$ DS, $1 \%$ immunoglobulin free BSA, $0.1 \%$

TritonX 100 in PBS 
Calcium-magnesium-free (CMF) medium: Hanks balanced salt solution + sodium bicarbonate

DAPI solution: $1 \mu \mathrm{g} / \mathrm{ml}$ DAPI in PBS

Dot blot sample buffer: $150 \mathrm{mM} \mathrm{NaCl}, 0.5 \mu \mathrm{M}$ EDTA, 10\% glycerol, 2\% SDS, $0.2 \mathrm{M}$ Tris Dot blot washing buffer: $0.2 \mathrm{M}$ Tris, $0.1 \%$ SDS

Eosin Stock Solution: $1 \%$ Eosin in $80 \%$ EtOH

ECL-solution 1: $10 \mu \mathrm{L} / \mathrm{mL} 250 \mathrm{mM}$ luminol, $4.4 \mu \mathrm{L} / \mathrm{mL} 90 \mathrm{mM}$ p-cumaric acid, 100 $\mu \mathrm{L} / \mathrm{mL} 1 \mathrm{M}$ Tris $\mathrm{pH} 8.5$ in distilled $\mathrm{H}_{2} \mathrm{O}$

ECL-solution 2: $0.9 \mu \mathrm{L} / \mathrm{ml} 30 \% \mathrm{H}_{2} \mathrm{O}_{2}, 100 \mu \mathrm{l} 1 \mathrm{M}$ Tris $\mathrm{pH} 8.5$ in distilled $\mathrm{H}_{2} \mathrm{O}$

Electrophoresis buffer for agarose gels (TAE): $40 \mathrm{mM}$ Tris (pH 7.6), $20 \mathrm{mM}$ acetic acid, 1 mM EDTA

Homogenization buffer for proteinase K: $0.05 \mathrm{M}$ Tris hydrochloride (pH 7.5), $0.15 \mathrm{M}$ $\mathrm{NaCl}, 5 \mathrm{mM}$ EDTA

Mowiol: $6 \mathrm{~g}$ Glycerin, $2.4 \mathrm{~g}$ mowiol, $6 \mathrm{ml} \mathrm{H}{ }_{2} \mathrm{O}, 12 \mathrm{ml} 0.2 \mathrm{M}$ Tris $\mathrm{pH} 7.2$

PMNs medium: culture medium containing DMEMF-12, supplemented with 2.5 $\mathrm{mg} / \mathrm{mL}$ BSA (35\%), 0.9\% D-(+)-glucose solution (45\%), $2 \mathrm{mM}$ Lglutamine, $5 \mu \mathrm{g} / \mathrm{mL}$ insulin, 1:100 N1 medium supplement, and 1:100 PSN antibiotic mixture

PBS: $9.55 \mathrm{~g} / \mathrm{L}$ pre-mixed PBS salt solution in distilled $\mathrm{H}_{2} \mathrm{O}$

PFA solution (4\%): for one liter $40 \mathrm{~g}$ PFA, $9.55 \mathrm{~g}$ pre-mixed PBS salt solution, 1-3 pellets of $\mathrm{NaOH}$ in distilled $\mathrm{H}_{2} \mathrm{O}$. Warmed up to $50{ }^{\circ} \mathrm{C}$ to ease solubilization while stirring. Adjust the $\mathrm{pH}$ to 7.4 at $4{ }^{\circ} \mathrm{C}$.

Re-suspension buffer for proteinase K digestion: in $0.05 \mathrm{M}$ Tris hydrochloride $(\mathrm{pH} 7.5)$, $0.15 \mathrm{M} \mathrm{NaCl}, 0.2 \%$ Sarkosyl

Running gel: $4.2 \mathrm{ml} \mathrm{30 \%} \mathrm{acrylamid} \mathrm{bisacryl,} 2.125 \mathrm{ml}$ 4x Tris pH 8.8, $2.125 \mathrm{ml}$ distilled $\mathrm{H}_{2} \mathrm{O}, 4.25 \mu \mathrm{l}$ TEMED, $42.5 \mu \mathrm{l}$ 10\% APS

SDS-page running buffer: $25 \mathrm{mM}$ Tris, $192 \mathrm{mM}$ glycine, 0.1\% SDS in distilled $\mathrm{H}_{2} \mathrm{O}$ Stacking phase gel: $0.65 \mathrm{ml} \mathrm{30 \%} \mathrm{acrylamid} \mathrm{bisacryl,} 1.25 \mathrm{ml} 4 \mathrm{x}$ Tris $\mathrm{pH}$ 6.8, $3.05 \mathrm{ml}$ distilled $\mathrm{H}_{2} \mathrm{O}, 5 \mu \mathrm{l}$ TEMED, $25 \mu \mathrm{l}$ 10\% APS 
TBS-T: $10 \mathrm{mM}$ Tris HCL, $150 \mathrm{mM} \mathrm{NaCl}$, 0.1\% Tween20 in TBS, pH 7.6 in distilled water Transfer buffer: 192 mM Glycine, 20\% Methanol, 25 mM Tris- $\mathrm{HCl}, \mathrm{pH} 8.3$

Tris: $10 \mathrm{mM}$ Tris-buffered saline $\mathrm{pH} 8.0$

Trypsin solution: trypsin $0.25 \mathrm{X}$ in sterile PBS solution

Washing solution for ICC: $2.5 \%$ BSA in PBS

\subsubsection{Animals}

The mouse strain B6;C3-Tg(Prnp-SNCA*A53T)83Vle/J (in this thesis addressed only as A53T mice) was initially purchased from Jackson Laboratories inc. and then the colony was increased through breeding at the "Zentrale Tierexperimentelle Einrichtung" (ZTE) facility of the university of Göttingen.

The experiments on the A53T mice were performed under the grant approval no. $13 / 1233$ and were carried out according to the regulations of the local animal research council and legislation of the State of Lower Saxony (Braunschweig, Germany).

The primary midbrain neuron cell cultures came from E-14 embryos of Wistar rats bred in the ZTE facility or purchased from Charles River. 


\subsubsection{Human tissue}

The samples used for X-ray diffraction and X-ray fluorescence experiments as well as for Particle induced X-ray emission were snap-frozen tissue blocks from SN region coming from the UK brain bank. Multicenter Research Ethics Committee approval (07/MRE09/72).

\begin{tabular}{|cccccccccc|}
\hline $\mathbf{N}^{\circ}$ & Age & Gender & $\begin{array}{c}\text { Onset } \\
\text { age }\end{array}$ & PMI & NPD & $\begin{array}{c}\text { Braak } \\
\text { staging }\end{array}$ & $\begin{array}{c}\text { Clinical } \\
\text { death from } \\
\text { clinical notes }\end{array}$ & Use \\
\hline PD029 & 76 & M & 70 & 15 & LBDBS & 6 & PD & $\begin{array}{c}\text { Multiple } \\
\text { microinfarts }\end{array}$ & PIXE \\
\hline PD102 & 81 & M & 56 & 16 & LBDBS & 5 & PDD & Infarct & $\begin{array}{c}\text { PIXE } \\
\text { XRD }\end{array}$ \\
\hline MS506 & 61 & F & 30 & 21 & CMS & - & MS & Pneumonia & PIXE \\
\hline MS517 & 48 & F & 23 & 12 & CMS & - & MS & Chest sepsis & PIXE \\
\hline MS532 & 68 & M & 29 & 14 & CMS & - & MS & Urosepsis & XRD \\
\hline
\end{tabular}

Table 1 Details of patients and control individuals who donated their brain to the UK brain bank that were used for this thesis. $P M I=$ post-mortem interval; $N P D=$ neuropathological diagnosis; aSYN score $=\alpha$-synuclein Braak stage; $C M S$ = conventional multiple sclerosis; $L B D B S=$ Lewy body disease brainstem variant; $L B D L=$ Lewy body disease limbic variant; $L B D N=$ Lewy body disease neocortical variant; $M S=$ Multiple Sclerosis; $P D=$ Parkinson's disease; $P D D=$ Parkinson's disease with dementia. The column "use" explains which samples underwent the analysis. $P I X E=$ sample analyzed with particle induced $X$-ray emission; $X R D=$ sample analyzed with $X$-ray diffraction.

\subsubsection{Facilities}

PIXE: The Particle Induced X-ray emission (PIXE) experiments were carried out in France at the Centre Etudes Nucléaires de Bordeaux Gradignan (CENBG, Bordeaux, France) using the platform AFIRA. The facility uses a Singletron as electrostatic accelerator. The experiments were done in collaboration with the group of Chemical Imaging and Speciation of Dr. Richard Ortega.

X-ray diffraction: The experiments were done in collaboration with the group of Prof. Dr. Tim Salditt at the Institute for X-Ray Physics, University of Göttingen. The x-ray diffraction experiments for the H4 cells were carried out at the P10 (Petralll, DESY, Hamburg) using the Göttingen Instrument for Nano-Imaging with X-rays (GINIX) setup as well as the X-ray tomography experiments on human tissue while the experiments with the human samples sections were carried out at the ID13 beamline of European Synchrotron Radiation Facility in Grenoble (France). 


\subsubsection{Vectors}

Plasmids: The $\mathrm{H} 4$ cell line was transfected with 2 plasmids simultaneously, courtesy of the group of Prof. T. Outeiro (Lazaro et al. 2014). Both plasmids used the pCDNA3 as backbone and the proteins were expressed under the CMV promoter. The SynT plasmid contained the sequence of human aSyn fused with the 93 aa from the EGFP sequence. The SNCAIP plasmid bears the sequence coding for Synphillin-1 with an attached V5 tag for ICC revelation of the protein.

Viruses: PMNs for the PIXE experiments were transfected with adenoassociated viruses AAV1/2. EGFP.ASYN; at a concentration of $1 \times 10^{8}$ i.u. that expressed WT aSyn and EGFP or AAV1/2.EGFP at $5 \times 10^{7}$ i.u. as control that expressed only EGFP as published (Ortega et al. 2015).

PMNs for the assessment of metal trafficking protein levels were transfected also with adeno associated viruses AAV1/2.MCHERRY.ASYN or AAV1/2.MCHERRY. The first virus expressed aSyn and the reporter protein mCherry under the control of the synapsin promoter while the second one was expressing only mCherry under the same promoter. Three different amount were used for both viruses: $3 \times 10^{7}, 1 \times 10^{8}$, $5 \times 10^{8}$ i.u. per well.

\subsection{9. qPCR primer list}

\section{ATP7a}

Primer Fw:
Accesion no.

Primer Rv:
NM_052803

CAGTGAACAGGGACATGCGA

GA

\begin{tabular}{llll}
\hline ATP7b & & Accesion no. & \multicolumn{1}{c}{ NM_012511 } \\
Primer Fw: & AGAGGCCAGTCGGAAAATCT & Primer Rv: & GGTAGTTGAGGATGAGCAGG \\
& TA & & T \\
& & Accesion no. & NM_133600.1 \\
CTR1 & CCCACGAGATGATGATGCCT & Primer Rv: & CACTGCCACAAAAGCTCCAG \\
& A & & \\
\hline DMT1 & & Accesion no. & NM_013173.2
\end{tabular}


Primer Fw: CAACGgAATAgGtGgagG Primer Rv: ACtGCCAACCCAAGTAGAAC ATC

AC

\begin{tabular}{llll}
\hline Fpn1 & & Accesion no. & NM_133315 \\
Primer Fw: & GAAGGATGCTGTGGATCTTT & Primer Rv: & CAAAGTGCCACATCCGATCC \\
& AGC & & \\
\hline TfR & & Accesion no. & NM_022712.1 \\
Primer Fw: & GGCCTATATGCTTGGGTAGG & Primer Rv: & GGCTCCCCTCCAAACAAGTTA \\
& AG & & \\
& & & \\
\hline
\end{tabular}

Table 2 List of primers used for qPCR for the detection of metal transporting proteins

Primers for GAPDH were purchased from Qiagen whose sequence is patented (\#249900).

\subsubsection{Software}

AxioVision 4.6 (Carl Zeiss Microimaging)

CatWalk XT 10.0 (Noldus)

CorelDRAW X3 (Corel Corporation)

EthoVision XT (Noldus)

GraphPad Prism 6.01 (GraphPad Software Inc.)

Gnu Manipulation Program - GIMP (The GIMP Development Team)

ImageJ 1.47v (N.I.H.)

Inkscape 0.91 (The Inkscape Team)

LAS AF 2.4.1 (Leica)

MATLAB R2013a Student Version (The MathWorks Inc.)

Mendeley (Elsevier)

PyMCA (Sourceforge.net)

Snapgene (GSL Biotech LLC)

SPSS (IMB Statistics) 
Stereo Investigator 9.0 (Micro Bright Field Inc)

\subsection{Methods}

\subsection{1. $\mathrm{H} 4$ cell culture experiments}

The $\mathrm{H} 4$ cell line was kept in culture and transfected as previously described (Klucken et al. 2004). The cells were cultured in Opti-MEM supplemented with $10 \%$ FCS and $1 \%$ pen/strep.

In order to perform the experiments $5 \mathrm{~mm} \mathrm{Si}_{3} \mathrm{~N}_{4}$ membranes were put into 24well plates and coated with $0.1 \mathrm{mg} / \mathrm{mL}$ of poly-D-lysine at $37^{\circ} \mathrm{C}$ overnight. Then membranes were washed in PBS 3 times and then 50,000 cells/well in $500 \mu \mathrm{L}$ of medium were seeded $24 \mathrm{~h}$ prior to transfection. One hour before transfection, the medium was removed and replaced with fresh medium without pen/strep (the antibiotics hamper the transfection). The transfection was accomplished according the protocol of FugeneHD by Promega. The ratio of transfecting agent to DNA was $3: 1$ and the ratio of the two plasmids synT/Synphillin-1 was 1:1. For each well, $2.5 \mu \mathrm{g}$ of total DNA were used (1.25 $\mu \mathrm{g}$ of each of the plasmid) and $7.5 \mu \mathrm{L}$ of transfection reagent; the remaining volume to $25 \mu \mathrm{L}$ was filled with Opti-MEM without antibiotics or FCS. The transfecting mixture was shaken by inversion several times and left in incubation $10 \mathrm{~min}$ before adding it to the cells. Three hours after the transfection the medium was changed and replaced with medium with pen/strep and serum.

$24 \mathrm{~h}$ post-transfections cells were fixated with 4\% PFA for $10 \mathrm{~min}$ at RT and stained. The staining was done permeabilizing the cells with $0.1 \%$ TritonX-100 in PBS for 20 min at RT. Then the Triton was removed and the blocking solution (1.5\% of NGS in PBS) was left $1 \mathrm{~h}$ in incubation. The primary antibodies were diluted into $1.5 \%$ NGS and left in incubation o/n. Anti-aSyn from BD and anti-V5 antibodies were used in a dilution of 1:1000 and 1:5000, respectively. The secondary antibodies, Cy2 goat anti-mouse for aSyn and Cy3 goat anti-rabbit for Synphillin-1, were diluted 1:1000 in NGS at RT for $2 \mathrm{~h}$. The nuclei were counterstained with DAPI for $5 \mathrm{~min}$. All the staining was done in the well in order to move the membrane the least possible. After this, a 
drop of PBS was put on the membrane and another Si-Ni membrane was placed ontop and sealed with nail polish and imaged.

After imaging, the membranes were put into a $1.5 \mathrm{~mL}$ vial with a hole on the cap for letting the air flow. Then the vials were put into liquid nitrogen and finally sent for imaging to P10 in Hamburg.

\subsubsection{Tissue preparation for $\mathrm{X}$-ray diffraction and $\mathrm{X}$-ray fluorescence and analysis}

The samples used were snap-frozen tissue blocks coming from the UK brain bank (see paragraph 2.1.6). For the imaging at ID13, we used sample PD102 and sample MS532.

Snap frozen tissue blocks from the midbrain of a PD patient and a MS patient (used as control) were cut $30 \mu \mathrm{m}$ thick in a cryostat at $-17^{\circ} \mathrm{C}$ with a sample holder at $-15{ }^{\circ} \mathrm{C}$ using special Teflon-coated blades (LEICA DB80 LX) to avoid iron particles contamination due to the friction. A special cryo-embedding matrix (LEICA 3801480 S) was employed as it was certified to be free from metal contamination.

After cutting, the sections were put either onto $\mathrm{Si}_{3} \mathrm{Ni}_{4}$ membranes (SILSON \#SiRN-12.5-200-8.0-500) or onto homemade $1 \mu \mathrm{m}$ thick polypropylene foils. The samples were then air dried for $15 \mathrm{~min}$ at RT and then placed into a drying oven for $30 \mathrm{~min}$ at $60^{\circ} \mathrm{C}$. Finally the samples were closed either with a Si-Ni membrane or with a polypropylene foil and sealed with nail polish.

For each section on the PP foil or on the $\mathrm{Si}_{3} \mathrm{Ni}_{4}$ membrane a consecutive section was cut and stained with hematoxylin and eosin (H\&E) stain to be used as a "guide".

$H \& E$ is one of the most common staining for histology as it can show nucleus and cytoplasmic inclusions (like LB) in clinical specimens (Dunnett \& Björklund 1999). It takes advantage of the combination of two dyes, hematoxylin and eosin. The hematoxylin gives to the nuclei a blue color upon the action of aluminum-based mordants, while eosin provides a pink stain for the cytoplasm and eosinophilic structures (Avwioro 2011). Due to their composition, upon staining, the LB in the brain tissue of patients result in rounded structures surrounded by a paler halo. In 
fact, eosin binds to acid amino-acid residues (like lysine and arginine) that are abundant in LB due to the aSyn presence (Morris \& Finke 2009).

H\&E staining was performed as previously described (Avwioro 2011) but without de-paraffinization as the tissue was snap-frozen. The tissue was directly immersed for 10 minutes in hemalum solution acidic according to Mayer (Roth, \#T865), rinsed in distilled water, and left under flowing tap water for 10 minutes. The stock solution of eosin (1\% of Eosin Y C.I. 45380 , in $80 \%$ EtOH) was diluted 1:5 in an $80 \%$ solution of EtOH with $50 \mu \mathrm{L}$ of acetic acid. The slices were incubated in the solution for 20 minutes, rinsed in distilled water, and left again under running tap water for 10 minutes. The sections were mounted with DePeX (Sigma \#06522).

Dried tissue and stained sections were imaged at the stereological microscope prior to X-ray imaging to be used during the beamtime as orientation.

The samples were imaged at the ID13 beamline of European Synchrotron Radiation Facility (ESRF) in Grenoble (France).

A scheme of the setup used can be found in Figure 2.1.
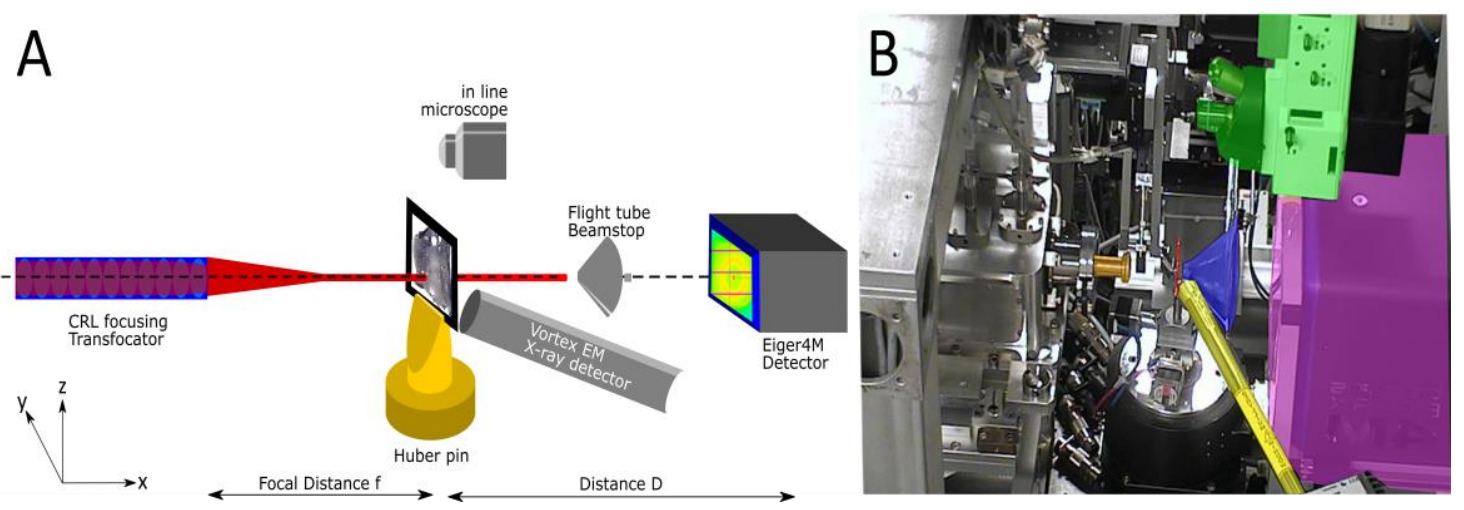

Figure 2.1 Experimental setup of ID13 experiments. A. Scheme of the setup at ID 13. B. in-line picture of the setup. Orange: focusing translocator; red: sample; yellow: Vortex EM X-ray detector; blue: beamstop/flight tube; green: in- line microscope; purple: Eiger $4 \mathrm{M}$ detector.

The samples were mounted on a Huber pin and the regions of interest were identified by searching with the in-line microscope. The X-ray diffraction patterns were recorded simultaneously with the X-ray fluorescence. The parameters used for the acquisition are illustrated in Table 3. 


\begin{aligned} & \hline Energy $[\mathrm{keV}] 13 \\ &$\hline Focal spot size $[\mu \mathrm{m} \times \mu \mathrm{m}] 2.7 \times 2 \\ &$\hline $\mathrm{I}_{0}[\mathrm{cps}] 9.9 \times 10^{11} \\ &$\hline Detector distance $[\mathrm{m}] 0.139$ (WAXS) \\ & \hline Focal distance $[\mathrm{mm}] 0.857$ (SAXS) \\ & \hline Detector Eiger 4M \\ & \hline Pixel $2070 \times 2167 \\ &$\hline Pixel size $[\mu \mathrm{m}] 75 \\ &$\hline Fluorescence detector Vortex EM X-ray detector \\ & \hline Energy resolution $[\mathrm{eV}]<130 \\ &$\hline Calibration Standards $\mathrm{Al}_{2} \mathrm{O} 3 / \mathrm{AgBh} \\ &$\hline\end{aligned}

Table 3 Experimental parameters and detectors used in the setup at ID13.

The experiment was conducted in collaboration with the group of Prof $\mathrm{T}$. Salditt. The data analysis of SAXS and WAXS patterns was done at the institute for Xray physics by J.D. Nicolas through Matlab scripts. The PyMCA evaluation of X-ray fluorescence data was done by me.

Bielschowsky silver stain: this stain was performed on deparaffinized $4 \mu \mathrm{m}$ thick sections as previously described (Yamaguchi et al. 1990). Briefly, the sections were put for 20 min into a $20 \% \mathrm{AgNO}_{3}$ solution; then shortly rinsed in water. The sections were incubated in the dark for $15 \mathrm{~min}$ in the same solution, but with the addition of some drops (ca. $2000 \mu \mathrm{L}$ ) of a $32 \%$ ammonia solution to the $20 \% \mathrm{AgNO}_{3}$ solution. Subsequently the sections were swung into an ammonia solution made of 3 drops (ca. $1000 \mu \mathrm{L}$ ) of the ammonia solution into a cuvette with $\mathrm{dH}_{2} \mathrm{O}$. Finally $3 \mathrm{~mL}$ of developing solution ( $12.5 \% \mathrm{PFA} ; 0.5 \%$ citric acid; $1 \% \mathrm{HNO}_{3}$ in water) were given to the ammonia- $\mathrm{AgNO}_{3}$ solution and let in incubation with the sections for $5 \mathrm{~min}$. The sections were rinsed in $\mathrm{dH}_{2} \mathrm{O}$ and placed for 2 minutes in a solution of $2 \%$ of $\mathrm{Na}_{2} \mathrm{~S}_{2} \mathrm{O}_{3}$. Ultimately the sections were washed with tap water, dehydrated through the ethanol series and mounted with non-aqueous medium. 


\subsubsection{Tissue preparation for particle induced $\mathrm{X}$-ray emission}

The samples used were snap-frozen tissue blocks coming from the UK brain bank (see paragraph 2.1.6). The imaging at AFIRA were done on blocs no. PD102, PD29, MS506 and MS517. The blocs used were cut similarly to the experiment in paragraph 2.2.2. For future orientation at the setup, pin-holes were inserted in the tissue punching it with a needle. The sections were cut $40 \mu \mathrm{m}$ thick in a cryostat at $17^{\circ} \mathrm{C}$ with a sample holder at $-15^{\circ} \mathrm{C}$ using special Teflon-coated blades (LEICA DB80 LX) to avoid iron particles contamination due to friction. The sections were then put onto a polycarbonate membranes that were kept inside the cryostat to avoid the thawing of the samples and the resulting displacement in metal compartmentalization. The unstained (native) sections were kept frozen in order to preserve at most the metal composition. Prior to imaging they were put into a lyophilizer for 3 days that removed the water and allowed the imaging at RT.

Adjacent sections were cut $40 \mu \mathrm{m}$ thick and placed onto microscope slides. They were fixated for $1 \mathrm{~h}$ with 4\% PFA then washed 3 times in PBS and lastly stained with H\&E.

The stained sections were imaged at the stereological microscope and searched for LBs. The position and the conformation of the LB on the adjacent slice, allowed the identification of possible LB in NM positive cells in the native sections that were analyzed through PIXE. The PIXE analysis was performed in Bordeaux (See Facilities section).

\subsubsection{Primary midbrain neurons culture preparation for particle induced $\mathrm{x}$-ray experiments and metal trafficking proteins}

Primary midbrain neuron (PMN) cultures were prepared as already described (Ortega et al. 2015; Knöferle et al. 2010). In brief, the brainstem was separated from E-14 embryos of Wistar rats and the meninges removed. At the ventricular opening of the tectum an incision was made and the rostral parts of the ventral brainstem and the tectum were cut away to isolate the mesencephalon floor. 
The whole dissection was carried out in ice-cold CMF medium. The collected tissue was centrifuged at RT for 4 minutes at $2.0 \mathrm{~g}$. Then the CMF was removed, and the tissue was incubated at $37{ }^{\circ} \mathrm{C}$ for $15 \mathrm{~min}$ with $1 \mathrm{~mL}$ of trypsin solution. The suspension was centrifuged for $2 \mathrm{~min}$ at RT to remove the trypsin. The remaining trypsin was inactivated with $1 \mathrm{~mL}$ of FCS and $50 \mu \mathrm{L}$ of DNAase solution were added. The tissue was then homogenized by gentle trituration trough a fire-polished glass Pasteur pipet. The cell suspension was centrifuged at RT for 4 minutes at $2.0 \mathrm{~g}$ and the FCS was replaced by medium. Cells were lastly counted by using a Neubauer counting chamber. Cells for PIXE were plated into polycarbonate holders inside 6-well plates at a density of 400000 cells/well while the same amount of cells for WBs and qPCRs were plated onto 24-well plates. The plates and the polycarbonate holders had been previously coated by poly-l-ornitine $(10 \mu \mathrm{g} / \mathrm{mL})$ for $3 \mathrm{~h}$ at RT then washed and a second coating with laminin $(10 \mu \mathrm{g} / \mathrm{mL})$ was applied for $16 \mathrm{~h}$ at $37^{\circ} \mathrm{C}$. This kind of cultures contained 5-10\% dopaminergic neurons and 90-95\% GABAergic neurons with very few glial fibrillary acidic protein-positive cells present (Lingor et al. 2000).

PMNs were left adhere to the support (plate or polycarbonate support) for 90 min. Then cultures to be analyzed with PIXE were transfected with adeno-associated viral (AAV) vectors to over-express wild-type human $\alpha$-synuclein and the reporter EGFP (AAV1/2.EGFP.ASYN; $5 \times 10^{7}$ p.f.u.) or only EGFP (AAV1/2.EGFP; $1 \times 10^{8}$ p.f.u.) as control (Ortega et al. 2015). At day 3 in vitro, PMNs were supplied with $50 \mu \mathrm{M} \mathrm{FeCl}_{2}$ for $4 \mathrm{~h}$ before cryofixation and freeze-drying.

The cultures to be analyzed for metal transporting proteins were transfected in the same way but with increasing amounts of AAV vectors: $3 \times 10^{7} ; 1 \times 10^{8} ; 5 \times 10^{8}$ either for the aSyn expressing virus (AAV1/2.MCHERRY.ASYN) or the Ctr virus (AAV1/2.MCHERRY). Cultures were then harvested on day in vitro 8 , because most of the metal transporting proteins are expressed in mature neurons.

\subsubsection{RNA isolation}

Cell lysis was performed according to the standard procedure for RNA extraction with TRI-reagent in order to harvest the neurons (Chomczynski \& Sacchi 1987; Chomczynski 1993). 
For a 24-well plate 3 wells/condition with about 500000 cells/well were used. The whole procedure was carried out under the fume hood to avoid toxic vapors. Cells were washed with ice-cold PBS once. $120 \mu \mathrm{L}$ of TRI Reagent were added in each well, subsequently $120 \mu \mathrm{L}$ more of TRI Reagent were supplemented. The TRI Reagent was left in incubation for $5 \mathrm{~min}$ at RT. The lysate was passed through the tip of a pipette several times to homogenize it. The lysate was collected in a $2 \mathrm{~mL}$ Eppendorf tube.

The phase separation was achieved by adding $200 \mu \mathrm{L}$ of 1-bromo-3chloropropane, and inverting the tubes 10 times. Then the samples were left $3 \mathrm{~min}$ at RT prior to centrifugation at $12000 \mathrm{~g}$ at $4{ }^{\circ} \mathrm{C}$ for $15 \mathrm{~min}$. The upper aqueous layer was collected as it contained the RNA and transferred into another tube. The bottom part containing proteins was saved for protein isolation for the WBs. The aqueous part was mixed well with $500 \mu \mathrm{L}$ of isopropanol and $1 \mu \mathrm{L}$ of glycol blue and then it was placed overnight into a $-20^{\circ} \mathrm{C}$ freezer to increase the yield. The Eppendorf tubes were then centrifuged at $12000 \mathrm{~g}$ at $4{ }^{\circ} \mathrm{C}$ for $30 \mathrm{~min}$. The pellet containing RNA was washed twice with $75 \%$ ice-cold ethanol twice by centrifuging $12000 \mathrm{~g}$ at $4{ }^{\circ} \mathrm{C}$ for 5 min. The pellet was then left to dry at RT for 5 minutes. The RNA was then dissolved in $30 \mu \mathrm{L}$ of RNA-free water and re-suspended using a vortexer and finally left at $55^{\circ} \mathrm{C}$ 10 min for complete dissolution. The RNA content was determined by UVphotometer (Nanodrop) at 230nm.

The samples were put in ice and underwent the reverse transcriptase reaction or stored in $\mathrm{a}-80^{\circ} \mathrm{C}$ freezer for long time storage.

\subsubsection{Reverse transcriptase reaction}

The reverse transcriptase reaction was conducted as specified in the Qiagen QuantiTect ${ }^{\circledR}$ protocol. Before proceeding with the real transcription reaction, the kit provides a DNA elimination reaction to reduce the presence of DNA contamination. For each reaction, $500 \mathrm{ng}$ of RNA were mixed with $2 \mu \mathrm{L}$ of genomic DNA wipeout buffer (in order to remove potential contamination due to the genomic DNA) to a total volume of $14 \mu \mathrm{L}$ that was reached by adding RNAse-free water. Each tube was placed at $42^{\circ} \mathrm{C}$ for 2 minutes to allow the reaction and then placed back on ice. 
After that, $1 \mu \mathrm{L}$ of Reverse Transcriptase was added to a mix containing the 14 $\mu \mathrm{L}$ coming from the previous reaction of the DNA elimination, $4 \mu \mathrm{L}$ of Quantiscript RT Buffer $5 \mathrm{x}$ and $1 \mu \mathrm{L}$ of RT Primer Mix in a total volume of $20 \mu \mathrm{L}$. The reaction was carried out at $42^{\circ} \mathrm{C}$ for 15 minutes. Subsequently an incubation of 3 minutes at $95^{\circ} \mathrm{C}$ served for the inactivation of Quantiscript Reverse Transcriptase.

The samples were put on ice and immediately analyzed through qPCR or stored in a $-80^{\circ} \mathrm{C}$ freezer for long time storage.

\subsubsection{Real time PCR after reverse transcriptase}

The real time PCR was performed according to the Qiagen protocol for SYBR green optimized for 96 well plates. Each qPCR reaction was prepared as in Table 4:

\begin{tabular}{lcc} 
Component & Concentration & Volume \\
SYBR green (2x mastermix) & $1 \mathrm{x}$ & $6 \mu \mathrm{L}$ \\
Primer Fw (10 $\mu \mathrm{M}$ stock solution) & $3 \mu \mathrm{M}$ & $0.36 \mu \mathrm{L}$ \\
Primer Rw (10 $\mu \mathrm{M}$ stock solution) & $3 \mu \mathrm{M}$ & $0.36 \mu \mathrm{L}$ \\
cDNA template & $31.25 \mathrm{ng}$ & $0.75 \mu \mathrm{L}$ \\
$\mathrm{H}_{2} \mathrm{O}$ for PCR & & $7.55 \mu \mathrm{L}$ \\
\hline Final volume & & $12 \mu \mathrm{L}$
\end{tabular}

Table 4 Components and amounts used for each single qPCR reaction.

For each target each reaction was made in triplicate. Every kind of cDNA sample was tested for all the targets mRNA including GAPDH as control.

The amplification was carried out according to the Qiagen protocol for SYBR green using a 3 step RT-PCR (Table 5): 


\begin{tabular}{lcc} 
Step & Time & Temperature \\
\hline Carryover prevention & $2 \mathrm{~min}$ & $50^{\circ} \mathrm{C}$ \\
\hline PCR activation step & $15 \mathrm{~min}$ & $95^{\circ} \mathrm{C}$ \\
\hline & 3 step cycling (repeated 40 times): & \\
\hline Denaturation & $15 \mathrm{sec}$ & $94^{\circ} \mathrm{C}$ \\
\hline Annealing & $30 \mathrm{sec}$ & $55^{\circ} \mathrm{C}$ \\
\hline Extension and data collection & $30 \mathrm{sec}$ & $72^{\circ} \mathrm{C}$
\end{tabular}

Table 5 Temperature protocol for qPCR for the quantification of the proteins responsible for metal trafficking.

The data were then analyzed by calculating the mean of the amplification Cts and then applying the formula of the fold-change by calculating $\Delta \Delta \mathrm{Ct}$ :

$$
\begin{aligned}
& \Delta \mathrm{Ct}_{\text {(target) }}=\mathrm{Ct}_{\text {(target) }}-\mathrm{Ct}_{\text {(GAPDH) }} \\
& \Delta \Delta \mathrm{Ct} \text { (target) }=\Delta \mathrm{Ct}_{\text {(aSyn) }}-\Delta \mathrm{Ct}_{(\mathrm{Ctr})} \\
& \text { Fold change }{ }_{\text {(target) }}=2^{\Delta \Delta \mathrm{Ct} \text { (target) }}
\end{aligned}
$$

\subsubsection{Protein purification after RNA extraction}

After the RNA extraction with the TRI-reagent, the protein purification was accomplished as previously described (Likhite \& Warawdekar 2011), always under the chemical fume hood because of the reagents toxicity. The proteins were contained in the organic phase of the TRI-reagent (bottom part, pink color). Before proceeding with protein purification, it was necessary to remove the remaining aqueous phase by pipetting. This method allowed also removing the DNA by precipitating it with $1 \mathrm{~mL}$ of absolute ethanol. Then the DNA was pulled down by centrifuging at $12000 \mathrm{~g}$ with a tabletop centrifuge for $30 \mathrm{~min}$.

The proteins were precipitated by adding $2 \mathrm{~mL}$ of $99.5 \%$ isopropanol. The protein pellet was washed 3 times with $2 \mathrm{~mL}$ of $0.3 \mathrm{M}$ guanidine hydrochloride in $95 \%$ ethanol. Lastly the pellet was left drying under the vacuum and re-suspended in a solution of $3 \mathrm{M}$ urea in Tris- $\mathrm{HCl}, \mathrm{pH} 8.0,1 \% \mathrm{SDS}, 1 \% \mathrm{Na}$-deoxycholate. 
The proteins were quantified with the Bicinchoninic Acid assay (BCA) according to the manufacturer protocol (Thermo Fisher). Bovine serum albumin (BSA) was used as standard for the calibration curve and the samples were read at $562 \mathrm{~nm}$ in a 96well plate reader.

\subsubsection{SDS gel electrophoresis}

The SDS gel electrophoresis was done in order to separate the proteins by molecular weight prior to western blotting as already described (Laemmli 1970). The gels were made using a separating gel at $10 \%$ and a stacking gel at $4 \%$ in polyacrylamide.

The separating gel was polymerized as first into $1.5 \mathrm{~mm}$ glass plates and it was prepared like described above (see 2.1.4). Then also the stacking gel was added. The gels were placed into the electrophoresis chambers and $40 \mathrm{mg}$ of total protein extracts were loaded into each well in order to detect the metal transporting proteins in the PMNs. As reference for the molecular weight of the proteins also the Dual color protein bench mark (biorad) was run altogether.

The gels were run at constant current of $20 \mathrm{~mA}$ for each gel until the line bromophenol blue contained in the loading buffer reached the bottom of the gel. Bromophenol blue runs together the with 5kDa proteins.

\subsubsection{Western blots}

After the SDS page electrophoresis, the proteins were transferred into PVDF membranes by using a Mini Trans-Blot Cell cassette. The PVDF was activated prior to use by submerging it into methanol for 30 seconds. Then the "transfer sandwich" was done by keeping the gel in contact with the PVDF membrane and adding two sheets whatman filter paper and a sponge that had been previously soaked up in the transfer buffer for each side.

The transfer was carried out at $100 \mathrm{~V}$ for $2 \mathrm{~h}$ at $4^{\circ} \mathrm{C}$ in order to prevent the proteins denaturation because of the heat due to the Joule effect. 
After the transfer, the membrane was incubated in blocking solution for $1 \mathrm{~h}$ at RT with $5 \%$ of skimmed milk in TBST. This step was necessary to block unspecific binding of the $A b$ with the membrane. Then the membranes were cut prior to the incubation with the primary antibodies as the expected weight of the proteins are different. All the $A b$ were diluted in $2.5 \%$ skimmed milk in TBST and left in incubation overnight at $4^{\circ} \mathrm{C}$ under agitation. $A$ list of the $A b$ is reported below in Table 6:

\begin{tabular}{llll} 
Protein & Host & dilution & Expected weight \\
\hline aSyn & Mouse & $1: 1000$ & $15 \mathrm{kDa}$ \\
\hline ATP7a & Rabbit & $1: 100$ & $163 \mathrm{kDa}$ \\
\hline ATP7b & Rabbit & $1: 100$ & $165 \mathrm{kDa}$ \\
\hline Ctr1 & Rabbit & $1: 200$ & $68 \mathrm{kDa}$ (tetramer) \\
\hline DMT1 & Rabbit & $1: 100$ & $55 \mathrm{kDa}$ \\
\hline Fpn1 & Rabbit & $1: 1000$ & $62 \mathrm{kDa}$ \\
\hline GAPDH & Mouse & $1: 5000$ & $37 \mathrm{kDa}$
\end{tabular}

Table 6 Antibodies used for the present thesis with their host, the dilutions applied and their expected weight.

The membranes were washed 5 times for 10 minutes with TBST. Then the secondary Ab conjugated horseradish peroxidase (anti-mouse or anti-rabbit see the table above) were left $1 \mathrm{~h}$ in incubation with a dilution of 1:2000. After another 5 times $\times 10$ minutes washes with TBST, the membranes were incubated for 1 minute with $1 \mathrm{~mL}$ of the ECL-solution 1 and $1 \mathrm{~mL}$ of the ECL-solution 2 to exploit the chemiluminescent reaction.

Then the membranes were put into an autoradiography cassette where the detection was achieved by exposing autoradiography films for different times in the dark room. Finally the films were developed into the developing machine.

The evaluation of the western blot results was done with ImageJ, taking into account the intensities of the bands of the metal transporting proteins compared to the intensity of GAPDH as a loading control. The statistics applied were ANOVA and Tukey test as post hoc. 


\subsubsection{Iron intoxication protocol and chelator treatment}

The transgenic murine line B6;C3-Tg(Prnp-SNCA*A53T)83Vle/J (short: A53T mice) from Jackson Labs (J004479; Bar Harbor, ME) was kept and bred within the Central Animal Care Unit of the University Medicine Göttingen, Germany. The animals were treated according to the regulations of the local animal research council and legislation of the State of Lower Saxony, Germany (33.12-42502-08-13/1233).

Animals were treated according to the protocol from (Fredriksson et al. 1999). Briefly, $40 \mathrm{mg} / \mathrm{kg}$ bw of Fe-carbonyl dispersed in $5 \%$ solution of sorbitol were given daily to the mouse pups between postnatal day (P) 10 and 17 through oral gavage tubes (Instech Laboratories, $22 \mathrm{ga}, 25 \mathrm{~mm}$ ). The concentration of Fe-carbonyl was 4 $\mathrm{mg} / \mathrm{mL}$ so that animals received a gavage volume that was $<=1 \%$ of their body weight. Even if the genotyping was done prior to oral gavage and the animals were tattooed, whole litters were gavaged in order to enhance the survival probably of the treated pups. Controls $(\mathrm{Ctr}$ ) received $5 \%$ sorbitol solution in the same period.

The oral chelator deferiprone (DFP, by Apopharma) was given at $10 \mathrm{mg} / \mathrm{kg}$ bw/day in the drinking water starting from $\mathrm{P} 100$ until sacrifice.

Animals were weighed weekly to ensure a check of the general conditions of health and also to monitor the onset of the disease, since A53T animals lose weight at this time point (Giasson et al. 2002).

The behavioral tests were performed as schematized in Figure 2.2. Catwalk and Novel object recognition test (NOR) were carried out at P 250 and at disease onset. The Rotarod was done weekly starting from P 220 until sacrifice.

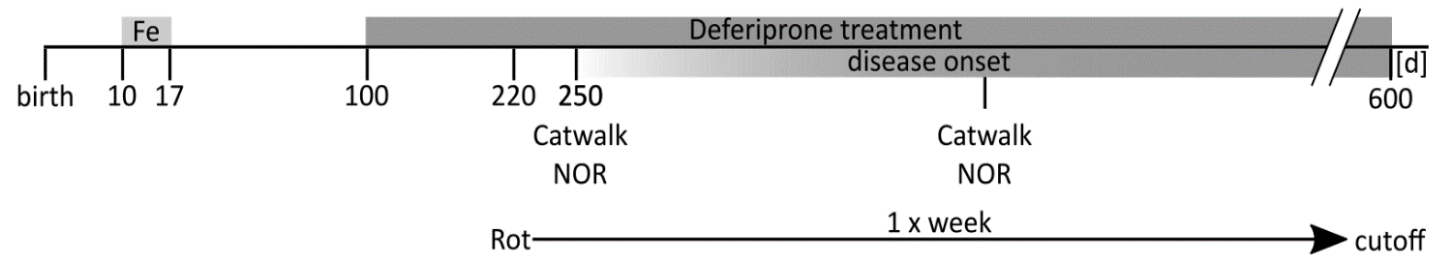

Figure 2.2 Time plan of the A53T mouse model with Fe supply. The pups were treated between $P 10$ and $P 17$ with $40 \mathrm{mg} / \mathrm{kg}$ bw of Fe-Carbonyl. At P $10010 \mathrm{mg} / \mathrm{kg}$ bw of the iron chelator DFP was given to the animals in the drinking water until sacrifice. Behavioral evaluations comprised Rotarod from $P 220$ until sacrifice; novel object recognition test and Catwalk at $P 250$ and at disease onset. 
The animals were divided into 4 different treatment groups:

- Ctr: A53T TG animals that received only oral gavage with vehicle;

- Fe: A53T TG animals that received oral gavage with Fe-carbonyl;

- DFPctr: A53T TG animals that received only oral gavage with vehicle and DFP supplement in the drinking water;

- DFPFe: A53T TG animals that received only oral gavage with vehicle and DFP supplement in the drinking water;

- WT Ctr: WT littermates that received only oral gavage with vehicle, they were used as controls.

\subsubsection{Genotyping of A53T TG mice}

The transgenic mice were maintained through heterozygous breeding pairs, because homozygous mice have a high incidence of nonproductive mating. The genotyping of the A53T animals was performed according to the protocol of the murine transgenic line supplier Jackson laboratories.

The genotype was performed prior to Fe intoxication; and animals were tattooed as early as postnatal day 2 . When the animals received the tattoo also a tail biopsy was taken to assess the genotype of each animal.

The tails were digested through the kit Direct PCR Lysis Reagent Tail (peqLab) as recommended by the manufacturer. Each tail was incubated overnight at $55^{\circ} \mathrm{C}$ with $200 \mu \mathrm{L}$ lysis buffer and $0.2 \mathrm{mg} / \mathrm{mL}$ Proteinase $\mathrm{K}$. The proteinase $\mathrm{K}$ was inactived by incubation at $95^{\circ} \mathrm{C}$ for 15 minutes.

Regular PCR: the regular PCR was used in order to sort out WT mice from heterozygous and homozygous for the transgene prior to the qPCR.

The primers had the following sequences (Table 7): 


\begin{tabular}{ll}
\hline TGT AGG CTC CAA AAC CAA GG & Primer Transgene forward \\
\hline TGT CAG GAT CCA CAG GCA TA & Primer Transgene reverse \\
\hline CTA GGC CAC AGA ATT GAA AGA TCT & Primer Internal Positive Control Forward \\
\hline GTA GGT GGA AAT TCT AGC ATC ATC C & Primer Internal Positive Control Reverse \\
\hline Table 7 Primers used for the genotyping of A53T mice. &
\end{tabular}

Each reaction was carried out as follows using Crimson polymerase (Table 8):

\section{Component}

Primer Transgene forward

Primer Transgene reverse

Primer Internal Positive Control Forward

Primer Internal Positive Control Reverse

Crimson buffer

dNTPs

$\mathrm{ddH}_{2} \mathrm{O}$

Crimson Taq

DNA template
Concentration Volume
$0.5 \mu \mathrm{M}$
$0.25 \mu \mathrm{l}$
$0.5 \mu \mathrm{M}$
$0.25 \mu \mathrm{l}$
$1 \mu \mathrm{M}$
$0.25 \mu \mathrm{l}$
$1 \mu \mathrm{M}$
$0.25 \mu \mathrm{l}$
$1 \mathrm{x}$
$4.00 \mu \mathrm{l}$

$0.2 \mathrm{mM}$

$2.00 \mu \mathrm{l}$

$11.87 \mu \mathrm{l}$

$0.03 \mathrm{U} / \mu \mathrm{L}$

$0.13 \mu \mathrm{l}$

$1.00 \mu \mathrm{l}$

$20 \mu \mathrm{l}$

Total volume

Table 8 Components and amounts used for each single qPCR reaction.

The PCR was done with the following protocol:

\begin{tabular}{lcc} 
Step & Time & Temperature \\
\hline Polymerase activation & $3 \mathrm{~min}$ & $94^{\circ} \mathrm{C}$
\end{tabular}

3 step cycling (repeated 12 times):

\begin{tabular}{lcc}
\hline Denaturation & $30 \mathrm{sec}$ & $94^{\circ} \mathrm{C}$ \\
\hline Annealing $\left(*-0.5^{\circ} \mathrm{C}\right.$ per cycle) & $15 \mathrm{sec}$ & $64^{\circ} \mathrm{C}$ \\
\hline Elongation & $35 \mathrm{sec}$ & $72^{\circ} \mathrm{C}$
\end{tabular}

3 step cycling (repeated 25times):

\begin{tabular}{lcc}
\hline Denaturation & $20 \mathrm{sec}$ & $94^{\circ} \mathrm{C}$ \\
\hline Annealing & $30 \mathrm{sec}$ & $58^{\circ} \mathrm{C}$ \\
\hline Elongation & $30 \mathrm{sec}$ & $72^{\circ} \mathrm{C}$ \\
\hline Last elongation & $2 \mathrm{~min}$ & $72^{\circ} \mathrm{C}$
\end{tabular}

Table 9 Temperature protocol for PCR for the genotyping of A53T mice. 
The PCR products were run at $100 \mathrm{~V}$ in a $1.5 \%$ gel in TAE for 45 minutes. The expected bands were of a single band for the internal control at $324 \mathrm{bp}$, and a $248 \mathrm{bp}$ if they carried the transgene. The presence of two bands meant the animal hat at least one copy of the transgene, while the presence of a single band meant it was a WT animal and it could be excluded from the qPCR reaction.

qPCR: once the WT animals were excluded from the genotyping, the qPCR was accomplished by using the following primers.

\begin{tabular}{llll}
\hline \multicolumn{1}{c}{ Primer } & 5' Label $^{\prime}$ & \multicolumn{1}{c}{ Sequence } & 3' Label \\
\hline $\begin{array}{l}\text { Internal Positive Control } \\
\text { Forward }\end{array}$ & & CAC GTG GGC TCC AGC ATT & \\
\hline $\begin{array}{l}\text { Internal Positive Control } \\
\text { Reverse }\end{array}$ & & TCA CCA GTC ATT TCT GCC TTT G & \\
\hline Transgene Forward & & TGA CGG GTG TGA CAG CAG TAG & \\
\hline Transgene Reverse & & CAG TGG CTG CTG CAA TG & \\
\hline Mutant probe & 6-FAM & CCC TGC TCC CTC CAC TGT CTT CTG & Zen probe with lowa \\
& & G & Black \\
\hline Internal control probe & Cy5 & CCA ATG GTC GGG CAC TGC TCA A & Black Hole Quencher 2 \\
\hline
\end{tabular}

Each reaction was carried out as follows using Biorad IQ Multiplex Powermix:

\begin{tabular}{lcc}
\multicolumn{1}{c}{ Component } & Concentration & Volume \\
Internal Positive Control & $40 \mu \mathrm{M}$ & $0.2 \mu \mathrm{l}$ \\
Forward & $40 \mu \mathrm{M}$ & $0.2 \mu \mathrm{l}$ \\
Internal Positive Control & $40 \mu \mathrm{M}$ & $0.04 \mu \mathrm{l}$ \\
Reverse & $40 \mu \mathrm{M}$ & $0.04 \mu \mathrm{l}$ \\
Transgene Forward & $5 \mu \mathrm{M}$ & $0.38 \mu \mathrm{l}$ \\
Transgene Reverse & $5 \mu \mathrm{M}$ & $0.65 \mu \mathrm{l}$ \\
Mutant probe & & $4 \mu \mathrm{l}$ \\
Internal control probe & $1 \mathrm{x}$ & $6.25 \mu \mathrm{l}$ \\
ddd ${ }_{2} \mathrm{O}$ & & $1.00 \mu \mathrm{l}$ \\
Biorad IQ Multiplex & & 12.76 \\
Powermix & & $\mu \mathrm{l}$ \\
DNA template & &
\end{tabular}

Each sample was done in technical triplicate. Every replicate consisted of 10.5 $\mu \mathrm{L}$ of the master mix solution in a 96-well micro plate. Positive controls for A53T TG 
homozygous and heterozygous animals coming from the Jackson laboratories as well as non-templates controls were also always added to the sample list.

The qPCR was done with the following protocol:

\begin{tabular}{lcc} 
Step & Time & Temperature \\
\hline Polymerase activation & $3 \mathrm{~min}$ & $95^{\circ} \mathrm{C}$ \\
\hline \multicolumn{2}{l}{2 step cycling (repeated 40 times): } \\
\hline Denaturation & $5 \mathrm{sec}$ & $95^{\circ} \mathrm{C}$ \\
\hline Annealing and elongation & $30 \mathrm{sec}$ & $60^{\circ} \mathrm{C}$
\end{tabular}

The qPCR machine calculated automatically the ratio between the signal of the TG controls and the internal controls, thus providing a relative amount of the copy regardless of the initial amount of DNA contained in the digested sample. In fact, heterozygous mice had only the half of the signal of the homozygous because heterozygous mice had only one copy of the gene, while the homozygous ones had two.

\subsubsection{Behavioral and functional tests: Rotarod}

Rotarod is a device that measures the balance, coordination and motor learning skills of rodents. It was performed as previously described elsewhere (Jones \& Roberts 1968)(Tatenhorst et al. 2016). The device employed was rotardod for mice by Ugo Basile (\#47600) where 5 mice could perform the test simultaneously (See Figure 2.3A). 
A

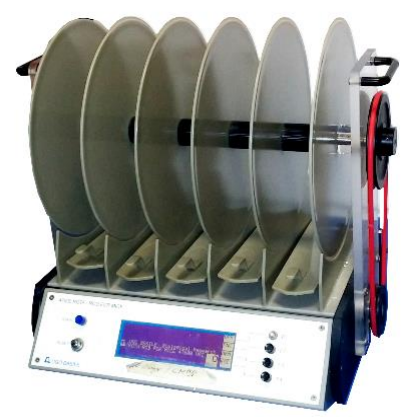

D

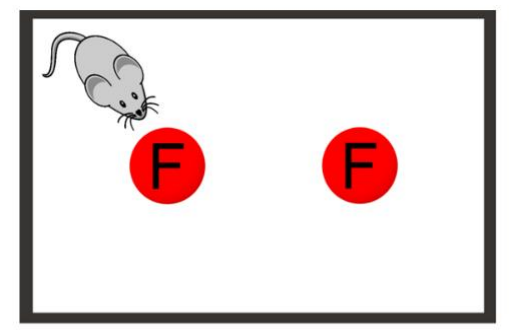

B

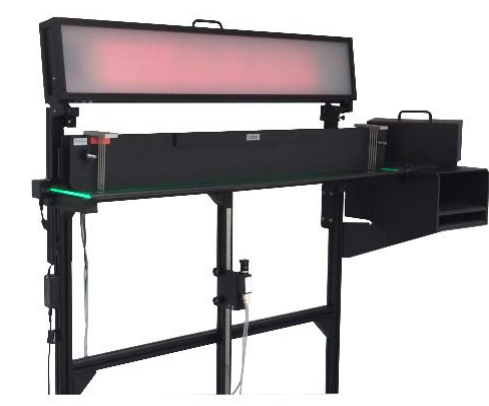

$\mathrm{E}$

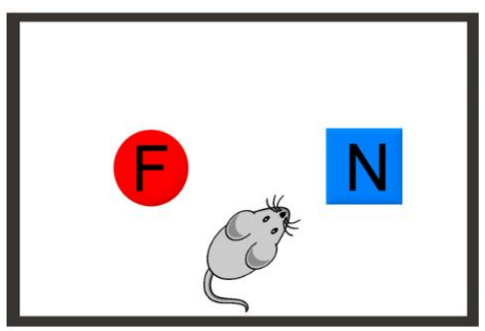

C

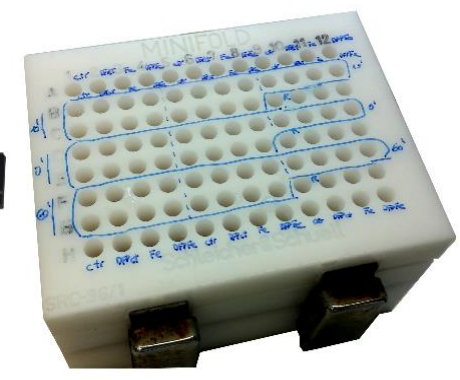

$\mathrm{F}$

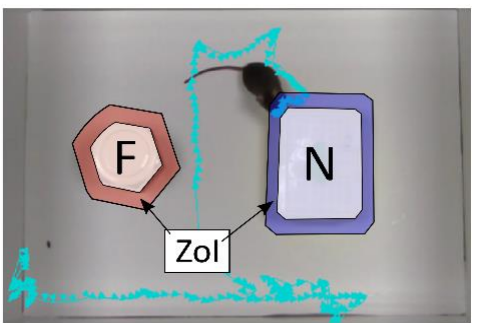

Figure 2.3 Scheme of the analysis performed on A53T animals. A. Rotarod device. In this study mice were running at accelerating speed from 5 to $40 \mathrm{rpm}$ for 300 seconds. B. CatWalk NT apparatus. The animals are placed inside the walkway with a glass floor where the camera below records the gait movements. C. Dot blot device. The proteins were placed onto the PVDF membrane through a vacuum force. D.E.F. Novel object recognition test. D. The animal is placed inside an arena with 2 identical objects in order to familiarize with them for 5 minutes. E. After 10 minutes back in the cage, the mouse was recorded for 5 min while exploring the arena with one new object and one familiar object. F. Screenshot of Ethovision XT 8.5. This software calculates the head direction to the object when the nose of the mouse is directed to the object and within $2 \mathrm{~cm}$ of its diameter, called zone of interest (Zol).

The animals were trained prior to real testing for 3 consecutive days on the device at $10 \mathrm{rpm}$ for 5 minutes. The recording was performed once a week from $\mathrm{P}$ 220 on. The device was set to accelerate ranging from 5 to $40 \mathrm{rpm}$ within $5 \mathrm{~min}$. Each test consisted of three repetitions. In order to reduce the stress and the fatigue the animals were left $30 \mathrm{~min}$ in the cage between the repetitions. Each weekly performance was evaluated as the average value of the three repetitions. The performance was recorded as the time (in seconds) spent on the rotating rod until the animal fell off or until the end of the task.

Rotarod was used to estimate the disease onset of the mice (Giasson et al. 2002). In fact, when the animals performed worse that the $50 \%$ of the long term average, this was considered as "disease onset". 


\subsubsection{Behavioral and functional tests: Novel object recognition (NOR) test}

The novel object recognition test is a test that assesses the presence of cognitive deficits in animals especially regarding memory and learning. The test was performed as previously described (Bevins \& Besheer 2006).

NOR takes advantage of the natural curiosity of mice and the fact that animals like exploring new things rather than familiar things. To that, a single mouse was placed for $3 \mathrm{~min}$ inside an arena of $48 \times 35 \mathrm{~cm}$ (habituation phase) to perform the NOR. Then two identical objects were put inside the arena like in Figure 2.3D, and the mouse was left for 5 minutes with the objects in order to familiarize with them. The mouse was inserted back for 10 minutes in its cage and subsequently placed in the arena for 5 minutes and recorded with one object that was the same of the familiarizing phase and a novel object Figure 2.3E. The recorded phase was also the step that was evaluated by using Ethovision XT 8.5 software by Noldus with blinding animal groups (see Figure 2.3F).

The software recognizes automatically the position of the nose of the animal tested taking this position as the head direction and is able to follow the movement of the mouse during the task (blue path on Figure 2.3F). Beside the two areas designed as novel object ( $\mathrm{N}$ in the figure) and familiar object ( $\mathrm{F}$ in the figure), also zones of interest (Zol) $2 \mathrm{~cm}$ all around the objects are taken into account as areas for exploration.

Ethovision can evaluate several parameters with such settings, such as the time spent with the familiar object and the novel object, the distance moved and the velocity. For our aims, the discrimination ratio was used and it was calculated as follows for all the groups:

Discrimination ratio $=$ time spent with novel object/(time spent with novel + old object) *100. 


\subsubsection{Behavioral and functional tests: Catwalk}

To assess the gait performance Catwalk XT gait analysis system (Noldus) was utilized. The test was performed at P 250 as well as at disease onset as described elsewhere (Saal et al. 2015; Tatenhorst et al. 2014).

The animals were place in a walkway with a glass bottom with a width of $4 \mathrm{~cm}$ and recorded by a high-speed digital camera placed below the glass. The glass was illuminated by a green light and from the ceiling of the walkway a red light gave a good contrast when the animals run on the camera (see Figure 2.3B).

The footprints were automatically recorded by a computer connected to the camera that was running the Catwalk XT 10.0 software. Detection settings were as follows: camera gain 20; intensity threshold 0.10; max. allowed speed variation $60 \%$. Three compliant runs per animal were recorded and means out of these runs were analyzed over treatment groups with Catwalk XT 10.0 gait analysis software.

The software allows the recordings and the calculation of up to 208 parameters. Therefore the outputs from the software were then statistically evaluated by selfwritten Matlab scripts for ANOVA and Tukey as post hoc test.

\subsubsection{Animal sacrifice and tissue processing}

At P 250 or when the animals showed signs of disease onset, animals were sacrificed by exsanguination.

The mice were deeply anesthetized by letting a flow of carbon dioxide inside a cage so that the air was slowly saturated by the gas and the animals did not undergo panic attacks. When the mice were unresponsive to toe pinches the carbon dioxide flow was stopped. The animals were secured in a supine position on a Styrofoam board by inserting pins in the paws.

Then the abdominal cavity was open by cutting along the thoracic midline with scissors two other cuts on the top and the bottom of the first cut were done in order to expose the thoracic ribs completely. Then the thoracic musculature was opened 
from the breastbone until the clavicles. The diaphragm was let open by inserting pins at the side of the animal. The pericardial sack was cut open and a needle connected with the perfusion pump was inserted in the beating heart inside the left ventricle. Then the flow of ice-cold PBS was started by turning on the peristaltic perfusion pump with a flow of $10 \mathrm{~mL} / \mathrm{min}$ and the right atrium was cut open to let the animal exsanguinate. The pump was switched off after 5 minutes.

The head was severed from the body and the skin around the skull removed. The skull was cut along the mid-sagittal suture and the side cranial bones were removed with forceps. The whole brain (including olfactory bulb) was removed from the cranial cavity and the two hemispheres were separated. One hemisphere was put into $4 \%$ PFA overnight to fixate for subsequent histological evaluations. The other hemisphere was put onto a $10 \mathrm{~cm}$ petri dish filled with ice and seven brain regions were removed under the stereological microscope for further biochemical evaluations (dot blots). The regions taken were: cortex, cerebellum, pons and medulla, olfactory bulb, hippocampus, midbrain and striatum. Immediately after dissections, the tissue was frozen by submerging the closed Eppendorfs in liquid nitrogen. The un-fixated tissue was stored at $-80^{\circ} \mathrm{C}$ until dot blot analysis.

\subsubsection{Proteinase $\mathrm{K}$ digestions and dot blots}

Proteinase $\mathrm{K}$ digestion: proteinase $\mathrm{K}$ digestion was carried out in order to assess the amount of aSyn aggregates in the post mortem brains of the A53T animals. Proteinase $\mathrm{K}$ is capable to digest the portions of proteins that are exposed to the solvent (e.g. native proteins) while leaving intact tightly packed proteins in the aggregates. The digestion was accomplished according to the protocol of (Butler et al. 1988).

The brain tissue was homogenate with four volumes of homogenization buffer at $4^{\circ} \mathrm{C}$ by using a bead homogenization machine (Precellys 24 Peqlab), then proteins were extracted by adding $1 \%$ Triton X-100 and $1 \%$ deoxycholate to the homogenates. A centrifugation at $5000 \mathrm{~g}$ for 10 minutes permitted the removal of nuclei and insoluble debris. The proteins in the supernatant were precipitated with 4 volumes 
of methanol overnight minutes at $-20^{\circ} \mathrm{C}$. The precipitate collected by centrifugation at $5000 \mathrm{~g}$ for 30 minutes.

The brains extracts were re-suspended in one volume of re-suspension buffer and the proteins quantified through BCA assay. To $22 \mathrm{mg}$ of total protein extracts 3.3 $\mu \mathrm{AU}$ of proteinase $\mathrm{K}$ were added adjusting the volume of $50 \mu \mathrm{L}$. Digestions were carried out at $37^{\circ} \mathrm{C}$ for 10 minutes and the reaction was stopped by adding $5 \mathrm{mM}$ PMSF that inhibits the proteinase K enzymatic activity. The samples were checked for the concentration again prior to addition with $5 x$ sample buffer. The samples were boiled at $95^{\circ} \mathrm{C}$ for 5 minutes and a subsequent dot blot was performed.

Dot blot: in order to visualize all the possible aggregated forms of aSyn, dot blots were performed as previously described (Oprandy et al. 1988). This method applies proteins directly onto a membrane by soaking them up with vacuum. The device used (Whatman Schleicher \& Schuell Minifold I by Sigma) is depicted in Figure 2.3C.

The PVDF membrane was activated by addition of methanol for 1 minute. Then the device was piled up according to the manufacturer instructions. Inside the compartment for the membranes of the device, two whatman filter papers were soaked up in washing buffer and they were piled up together with the PVDF membrane at the top. Then $400 \mu \mathrm{L}$ of washing buffer was left run through the vacuum in each of the 96-wells, to wash them and in order to help the rubbers at the bottom of the wells seal properly on the PVDF membrane.

$10 \mu \mathrm{g}$ of each sample were added with the sample buffer for the dot blot up to $400 \mu \mathrm{L}$ final volume and let run through the wells by applying vacuum. Finally, an additional $400 \mu \mathrm{L}$ of washing buffer were given to wash the wells. Then the membrane was blocked for one hour in 5\% dry skimmed milk in TBS-T. Then the primary anti-human aSyn antibody (Invitrogen 32-8100) was added to $2.5 \%$ in TBS-T. The revelation was done as previously described for the Western Blot. 


\subsubsection{Statistical analysis}

Statistical analysis were carried out taking (Emden et al. 2008) and the (Campbell 1989) as reference.

The software used for the analysis were Matlab 2013a Student Version (The MathWorks Inc.) together with GraphPad Prism 6.01 (GraphPad Software Inc.) for all the experiments listed here. For the Kaplan-Meier curve of the animal survival the software SPSS statistic 24 was used. For group comparisons ANOVA together with Tukey test as post-hoc test was applied.

The statistical test and the number of animals or experiments for each analysis are specified in the respective figure legends. Data are given as mean \pm SEM. Differences were considered significant with $\mathrm{p}<0.05\left(* \mathrm{p}<0.05\right.$; $\left.^{* *} \mathrm{p}<0.01 ; * * \mathrm{p}<0.001\right)$. 


\section{Results}

The results are divided into three main sections: the first part shows how alphasynuclein (aSyn) aggregates can be studied with biophysical techniques, the second how aSyn can vary the metal content in cells and the last part deals on how the supply of Fe can enhance the aggregation processes of aSyn in an in-vivo model of Parkinson's disease.

\subsection{Alpha-synuclein aggregation and $\mathrm{X}$-ray imaging}

This section describes the effort to visualize aSyn aggregation by using different biophysical imaging techniques. Particularly, two different aspects were investigated.

The first aspect was characterizing the aggregation in a cellular model by phase contrast $x$-ray imaging (PCXRI). The goal was to analyze the signal produced by aggregates in order to be able to identify possible pre-aggregated forms to study the evolution of the process. After that aggregates were imaged in the brains of real PD patients searching for comparable features. Therefore we went for an analysis of fresh-frozen brain tissue from the UK PD brain bank.

\subsubsection{Alpha-synuclein aggregation in $\mathrm{H} 4$ cells}

Neurotoxicity in PD appears to be exerted by oligomers of aSyn by yet not completely known mechanisms. The aim of this experiment was to characterize the aggregation of aSyn in a cellular model using phase contrast x-ray imaging (PCXRI). To this end, we took advantage of the model of aggregation in the $\mathrm{H} 4$ cell line of human neuroglioma with the co-transfection of two plasmids: SynT and Synphillin-1 (see Materials and Methods).

The examples of samples preparation and the results obtained are summarized in Figure 3.1. Cells were seeded at a very low concentration in order to have the possibility to image single cells (see Figure $3.1 \mathrm{~A}$.). 

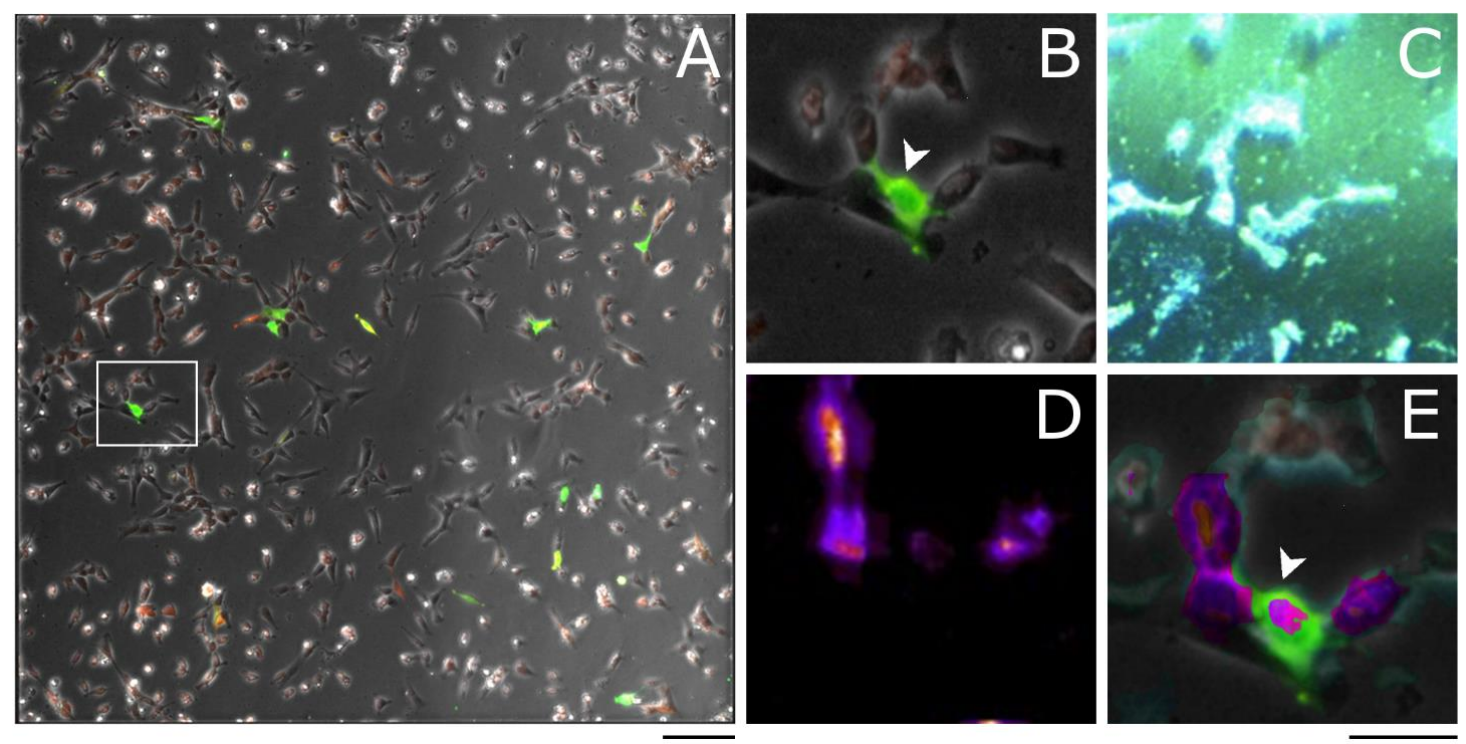

Figure 3.1: SynT/Synphylin-1 aggregation in H4 cells imaged by PCXRI. A. Photomicrograph of a whole Si3N4 membrane used for $x$-ray imaging after staining for SynT (green) and Synphylin-1 (red). Aggregates are yellow. The white highlight square represents the analyzed area Scale bar: $500 \mu \mathrm{m}$. B. Photomicrograph of the analyzed area before freeze-drying. The white arrow points towards aggregates in the cells. C. Online image of the analyzed area $D$. $X$-ray dark-field image of the corresponding area. E. overlay of the images in B. C. and D. The white arrow points to the aggregates. Scale bar: $20 \mu \mathrm{m}$

The white square depicts the area that was analyzed with X-rays. The same area is visible in the panel $B$, and here the white arrow points towards the aggregates (where there is co-localization between aSyn and Synphillin-1). The images in the panels $A$ and $B$ were taken at the epifluorescence microscope before the freezedrying procedure in order to avoid the fluorescence quenching that can happen at low temperatures.

The on-line microscopic image was taken as morphological reference for the cells (panel $\mathrm{C}$ in the figure). The freeze-drying procedure successfully retained the morphology of the cells as can be see comparing the cell morphology between the image in B and C. After that, the X-ray dark-field image of the analyzed area was acquired and the result is shown in the panel $D$. The image was taken with a Pilatus300k detector at $5 \mathrm{~m}$ distance, this distance allowed to collect data in the SAXS range. Since the $\mathrm{X}$ - ray dark-field image has been obtained by X-ray scattering due to the anisotropy of the imaged structures, the overlay in E implies that there is no clear polarization of the signal were the aggregates have been imaged (white arrow). The high signal of the neighboring cell (top left image) might be due to the fact that this cell could have been fixated during the condensation phase of apoptosis (the cell is 
round and with no processes) and therefore it might be arising from the presence of compacted DNA.

\subsubsection{X- ray diffraction on PD patients tissue}

PCXRI showed that there was no variation in the density contrast of the $\mathrm{H} 4$ cells where the immunocytochemistry pointed out the presence of aggregates. Therefore a comparison with human material from PD patients was necessary to understand whether this was characteristic of the employed cell culture model, or if this feature was similar to the tissue coming from patients with of Parkinson's disease and was therefore a peculiarity of aSyn itself.

Eight tissue sections from snap frozen blocks of SN were imaged at ID13 at the ESRF. Four samples were coming from a PD patient and four samples from a Ctr patient (see Chapter 2). The samples were analyzed for X-ray diffraction in the SAXS and WAXS range with a micro focus beam with a step size of $3 \mu \mathrm{m}$.
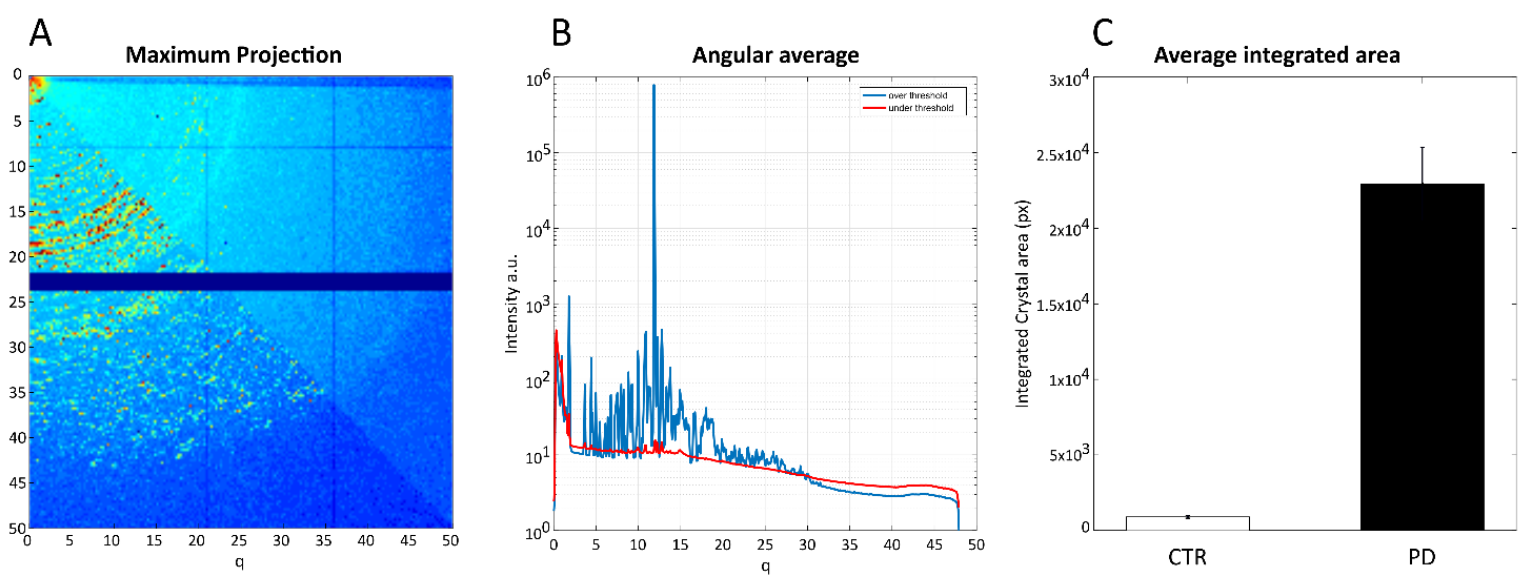

Figure 3.2. WAXS analysis of PD sample and CTR sample after thresholding. A. Maximum projection of the scans after thresholding for wide angles (threshold at $q=3$ ). The areas above threshold show a distinct pattern of protein crystals, while the areas under the threshold have a higher signal in the SAXS range. B. Angular average of the areas above threshold (blue) and under threshold (red). C. Average integrated area. The histogram shows the average number of pixels over the threshold for each scan for both samples. The crystals appear to be present mostly in the PD sample. $n=1$.

The goal was to characterize the X-ray diffraction signal coming from LB found with the H\&E stain in the adjacent section, but it was not possible to identify the same cells also in the native section. However an analysis on the scattering in the SAXS and 
WAXS was carried out on the tissue in the SNpc, SNpr and cerebral peduncle zones of PD patients and Ctr samples.

The scans showed an unexpected characteristic signal in the WAXS range, thus implying the presence of macromolecular highly ordered structures.

Not all the areas imaged displayed a WAXS signal. Therefore, the crystal mapping was made separating the SAXS scans from the WAXS scans by taking $q=5$ $\mathrm{nm}^{-1}$ as limit for the SAXS. Then a threshold intensity of 50 counts was used in order to discriminate between patterns above and below threshold in the WAXS range (see Figure 3.2). The maximum projection (depicted in the panel $A$ of the same figure) was obtained by summing all the signals coming from the areas above threshold (bottom left corned) and below the threshold (upper right corner). Looking at the comparison of the maximum projection of the different areas is possible to appreciate that the patterns are completely different.

The angular average (depicted in the panel B), implies that there are two characteristic peaks at about $1.7 \mathrm{~nm}$ and $3.3 \mathrm{~nm}$ that aren't present it the angular average below the threshold. Intriguingly, when the areas above threshold were summed according to which sample type, they revealed to be mostly present in the scans of PD samples (panel C). There was no statistical test run here because, even if the scanned sections were giving a clear indication about which kind of samples had the highly ordered structures, there was the opportunity to image only a single PD sample and a single Ctr sample therefore they represent technical replicates for statistical analysis.

In order elucidate what could cause the signal in the WAXS range, it was hypothesized that it could have been because of the presence of aggregated proteins. 

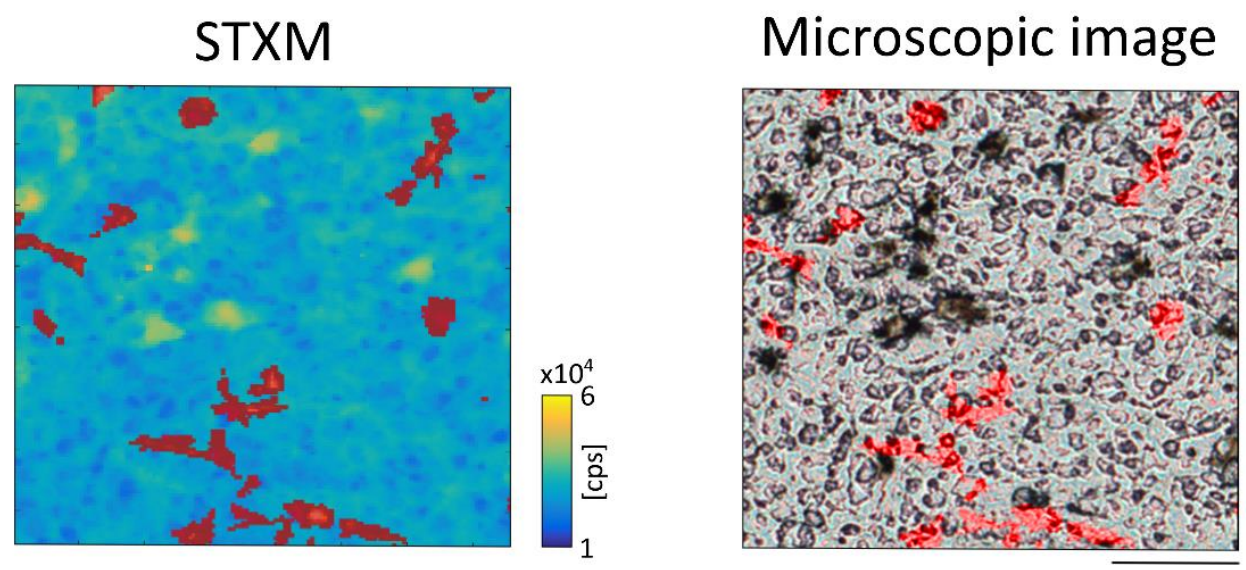

Figure 3.3 Spatial distribution of the areas with highly ordered structrues. The overlay red signal depicts the areas from which the WAXS signals arises. Left. The image of analyzed areas is done with the Scanning transmission X-ray microscopy (STXM). The yellow spots represents the densest areas that are NM positive cells. Right. Corresponding microscopic image of the analyzed region. Scale bar: $20 \mu \mathrm{m}$.

The localization of the areas with highly ordered structures was compared with the microscopic images and with the signals coming from the Scanning transmission X-ray microscopy (STXM) (6 scans for PD and 6 for Ctr). An example of the comparison can be seen in in Figure 3.3, where the red areas correspond to the areas with highly ordered structures in overlay with the STXDM image (Figure 3.3 left) and corresponding microscopic image (Figure 3.3 right) .

The STXM image displays with higher signals the areas that are most dense in the specimens. In this case, the Fe present inside the pigment neuromelanin (NM) is denser than everything else in the tissue due to the presence of $\mathrm{Fe}$, and hence it allows us to discriminate the NM positive cells. This feature is very useful to compare the SDXM image with the light microscopy image. The areas where the crystal structures were found, appear to be in the extracellular space in both the images in all the scans analyzed.

In order to understand which kind of aggregated protein could have been the origin of the signal of the crystals, the tissues were tested for the most common proteins that are known to aggregate in the brain (Irvine \& El-Agnaf 2008). In fact, sections from snap frozen blocs that were analyzed in the ESRF, were tested in IHC for aSyn, Amyloid-beta and p-Tau. Amyloid-beta and $p$-Tau were stain were negative, whereas aSyn stain proved to be homogenously diffused throughout the sections and 
there were no detectable aggregated forms in the cryo-sections tested coming from the PD sample and the Ctr sample.

Since our aim was the visualization of extracellular ordered proteins structures with a histological technique, the tissue blocs were embedded in paraffin and subsequently we performed a Bielschowsky silver stain on PD tissue compared to $\mathrm{Ctr}$ tissue (Figure 3.4). This stain illustrates the position of the axons together with amyloid formations such as aggregated Tau and Amyloid-beta (Uchihara 2007).
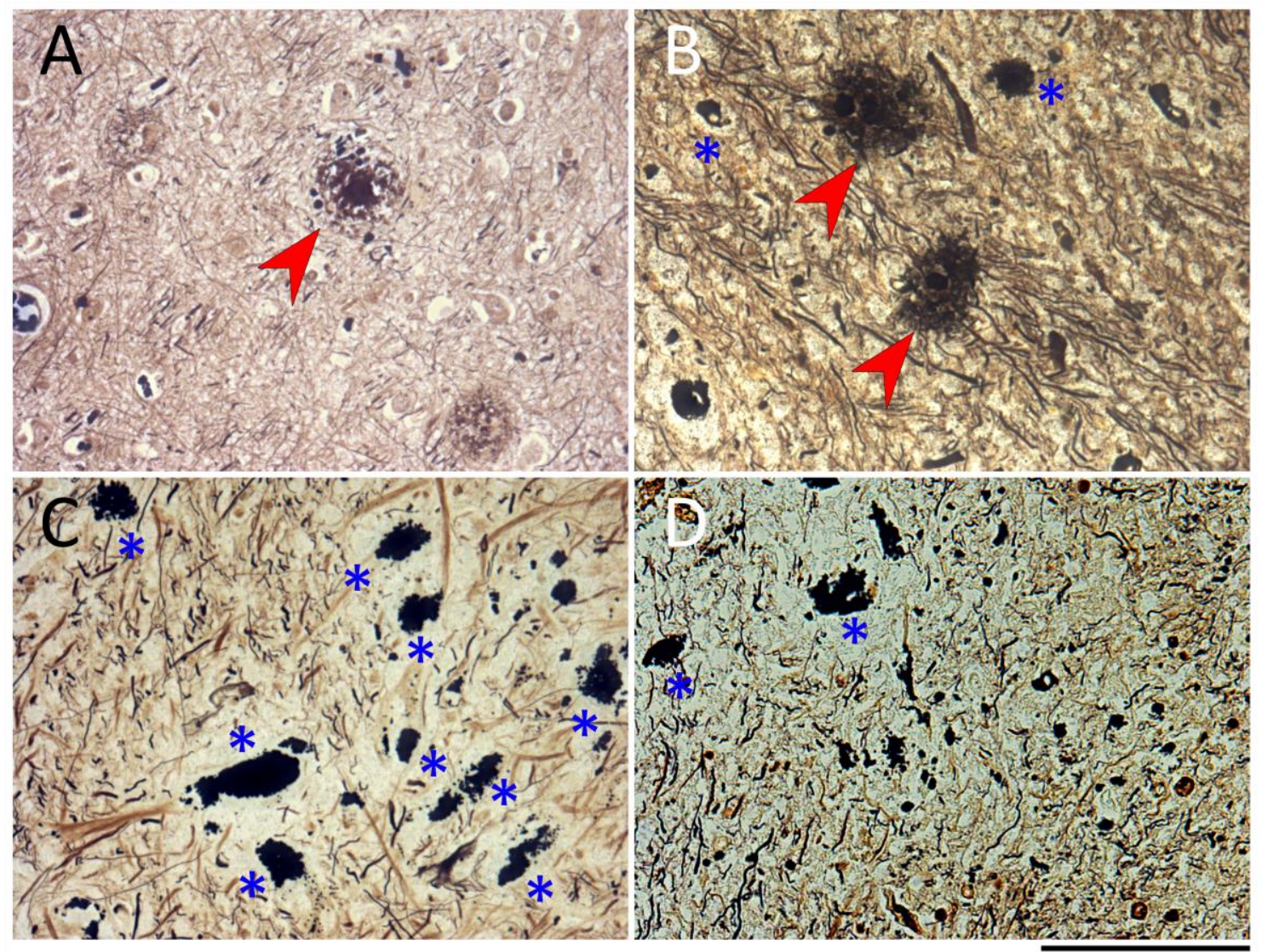

Figure 3.4 Histological evaluation of amyloid structure using Bielschowski silver stain. A. Positive control (from an Alzheimer's patient). The red arrows is pointing towards amyloid-positive structures. $B$. $S N p r$ of PD sample with positivity for amyloids structures (red arrows). C. Ctr sample SNpc. The dark cells are healthy NM positive cells (indicated with blue stars) D. PD patient sample SNpc. The NM positive cells (blue stars) are few and appear to be damaged or dying. Scale bar: $100 \mu \mathrm{m}$.

The histology demonstrated the presence of amyloid structures in the PD patient in the SNpr (see Figure 3.4 panel B) that are less pronounced than Alzheimer sample (positive control panel A). These structures are fiber-like and they appear to be in the extracellular space sharing some similarities with the distribution of the highly order aggregated areas. However, the morphology of the aggregates looks different between the staining and the WAXS data. Regarding the dimensions, the 
amyloid structures showed in the histological stain range from 20 to $60 \mu \mathrm{m}$ and the length of the distribution of the highly order structures ranges from 30 to $70 \mu \mathrm{m}$.

\subsubsection{X-ray fluorescence on PD patients tissue}

Assessing the metal content in PD tissue and particularly in the $\mathrm{SN}$, is an important topic in the field, especially in relationship to the presence of aSyn aggregates. In fact, it is very well known that aSyn aggregates better and faster in presence of various metals (among those $\mathrm{Cu}$ and Fe are the most prominent) (Uversky et al. 2001). Since aggregated forms of aSyn are found in the LBs of the SN of PD patients and represent a hallmark of the disease (Tofaris \& Spillantini 2007), understanding whether the metal can co-localize with aSyn, can provide deeper characterization of PD pathogenesis. Thus sections of this brain region were imaged at the ESRF.

Taking advantage of the set up at ID13, X-ray fluorescence (XRF) spectra of the samples described in paragraph Errore. L'origine riferimento non è stata trovata. were acquired simultaneously with the X-ray diffraction spectra. This had the advantage of combining a good spatial resolution of the X-ray diffraction together with the localization of the metals as readout of the XRF. The XRF is a technique that gives as readout the energy spectrum of elements. In biological samples this allows to calculate amounts of trace metals and their concentration (Cesareo 1976). It provides a quantitative analysis (relative to a surface unit) and also a spatial information about the elemental distribution of a given biological specimen.

It was not possible to perform a calibration of the instruments, thus we had to calculate the abundance of each element as a relative measure. During the analysis, the whole elemental spectra of all the scans were examined. $\mathrm{Zn}$ was chosen as the element in order to discriminate between intra and extracellular content, as it was the element with the greatest difference in concentration between extracellular and extracellular space (about the $75 \%$ of the whole $\mathrm{Zn}$ amount was contained in the cells). Zn was selected in order to compare intracellular and extracellular areas of the scans. Therefore, after setting a threshold for Zn, 4 different spectra were calculated: 
intracellular and extracellular content in the PD sample, and intracellular and extracellular content for the CTR sample.
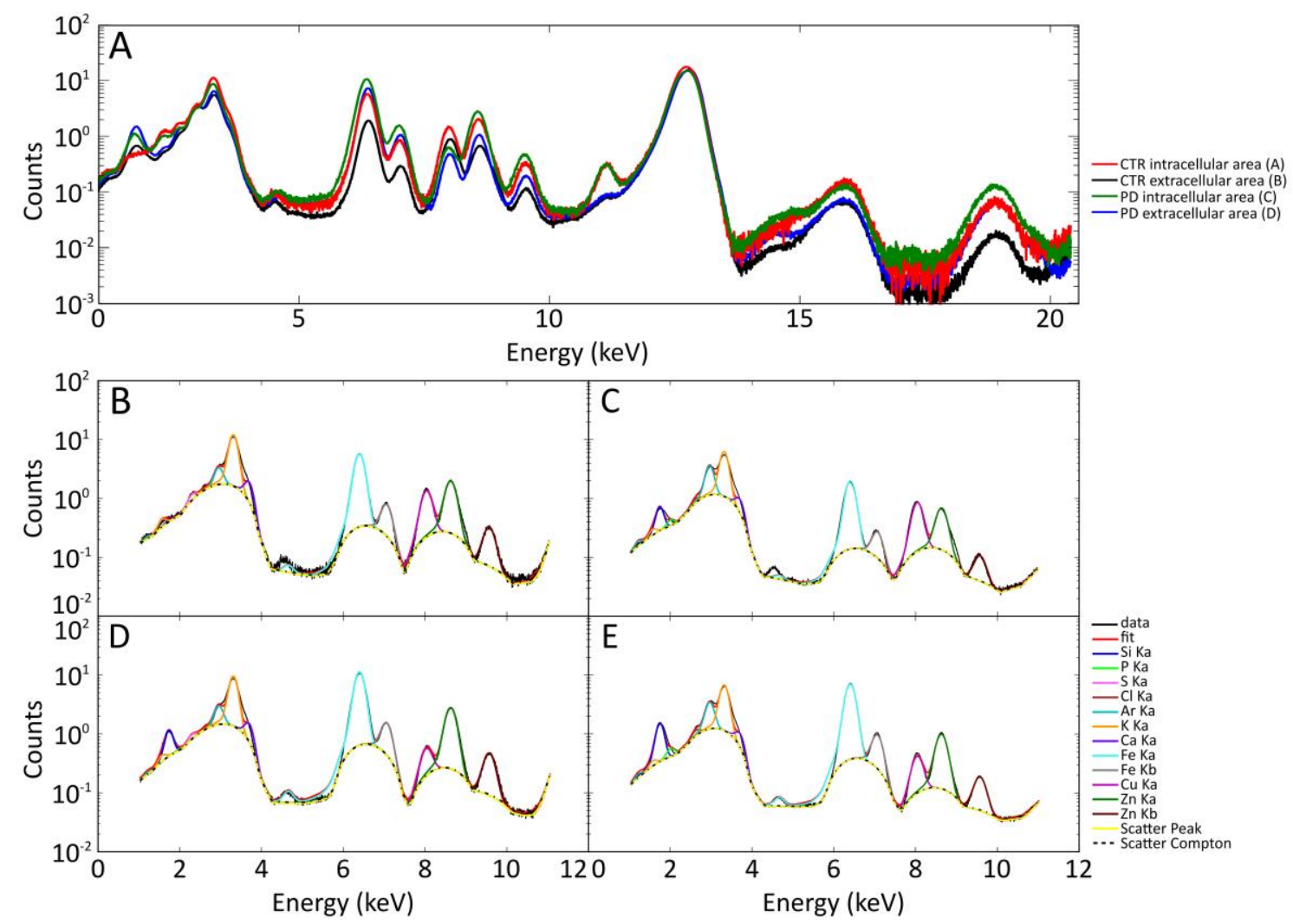

Figure 3.5 A. Overlay of the four fluorescence spectrum calculated. Bottom part. Curve fitting through PYMCA. B. CTR intracellular area, C. CTR extracellular area, D. PD intracellular area, E. PD extracellular

The program used for the calculations was PyMCA. This program allows the fitting of the elemental fluorescence curves and calculates the area under the curve for each element together with the S.E.M. (Figure 3.5). The program calculated the elements areas by fitting the peaks for Si Ka, P Ka, S Ka, Cl Ka, Ar Ka, Ca Ka, Fe Ka and $\mathrm{Kb}, \mathrm{Cu} \mathrm{Ka}, \mathrm{Zn} \mathrm{Ka}$ and Kb. The panels B, C, D and E show the overlay of the different elemental peaks on the real data acquired (black line).

From these areas, the relative concentration for each element was calculated as follows: $(P D-C T R) / C T R$ were the PD and CTR are the areas calculated for a given element. The results can be seen in Figure 3.6. 


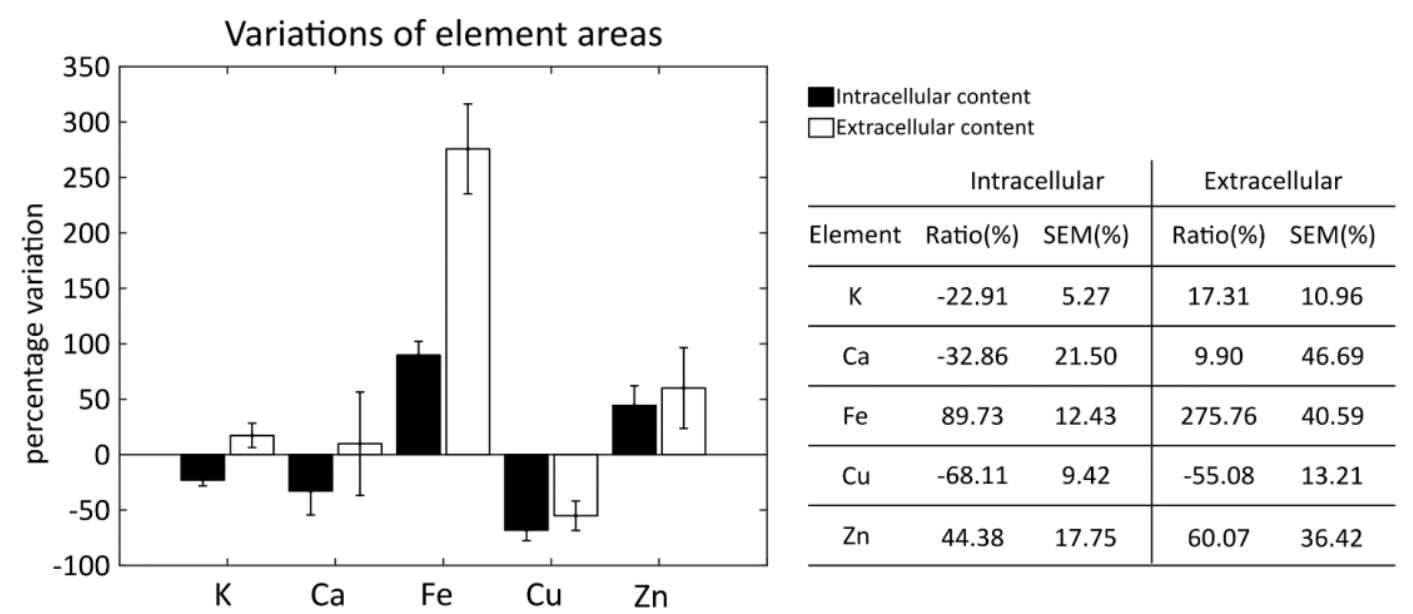

Figure 3.6 Relative elemental quantification of X-ray fluorescence from PD samples compared to Ctr. The samples were analyzed by using a $\mathrm{Zn}$-based threshold in order to discriminate between intracellular and extracellular content. The variation of the element concentration is calculated using the area under the curve of the elemental fitting. $n=1$

After the elemental analysis, it can be stated that PD samples displayed the $89.93 \pm 12.43 \%$ more Fe in the intracellular compartment when compared to Ctr, while the amount of Fe in the extracellular compartment was even $275.76 \pm 40.59 \%$ more that the Ctr. Even the $\mathrm{Zn}$ content appeared to be increased (respectively of $44.38 \pm 17.75 \%$ intracellularly and of $60.07 \pm 36.42 \%$ extracellularly when compared to the $\mathrm{CTR}$ ). On the other hand the amount of $\mathrm{Cu}$ is reduced both in the intracellular (68.11 $\pm 9.42 \%$ less compared to CTR) and extracellular spaces $(-55.08 \pm 13.21 \%)$.

It has been shown that in in-vitro conditions many proteins involved in neurodegenerative diseases (like amyloid-beta, aSyn and prion protein) have the tendency to aggregate in presence of metals like Fe and Cu (Breydo \& Uversky 2011). Therefore we compared the spatial distribution of the highly ordered structures shown in Figure 3.3 with the elemental maps of the respective areas in Figure 3.7 (taken as exemplary pictures). This was possible because of the set-up used that allows raster scans, therefore XRF not only provided a quantification of the trace elements, but also their spatial localization.

Six scans from the PD patient and six from the Ctr sample were analyzed to make this comparison. We looked at spatial distribution of the highly ordered structures comparing whether there was a co-localization between the metal distribution (total fluorescence, $\mathrm{Zn}, \mathrm{Cu}$ and Fe distribution) with the $\mathrm{Zn}$ fluorescence 
taken as reference for distinguishing the $\mathrm{Zn}$-rich neuromelanin positive cells from the background (Zecca et al. 1996) (Figure 3.7 central column) .

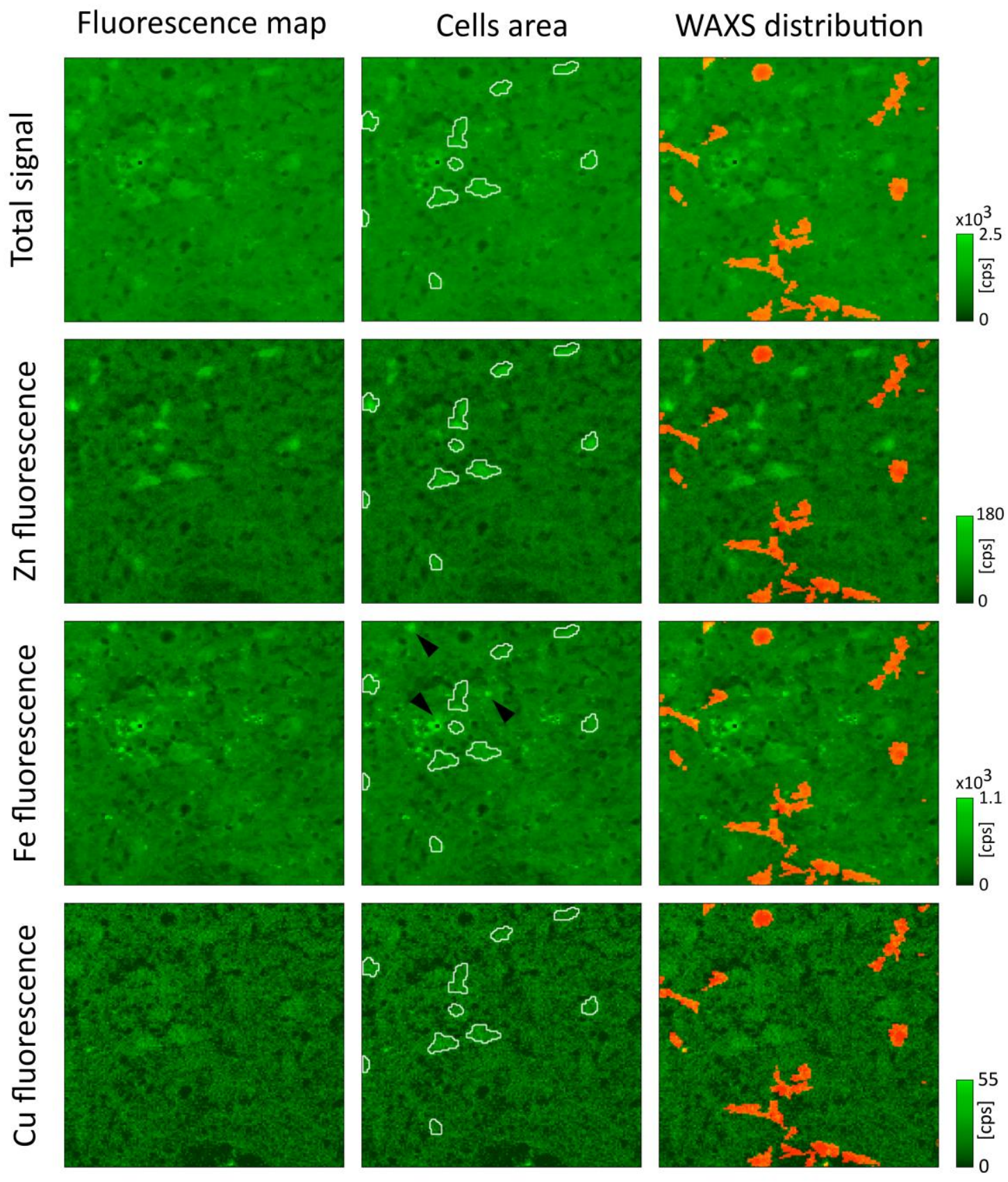

Figure 3.7 Example of total fluorescence maps and the maps for $\mathrm{Zn}$, Fe and $\mathrm{Cu}$. The maps are shown as a green scale. Overlay images of the areas recognized by the algorithm as cells (white contours) and of the areas where there was WAXS signals after thresholding (red overlay). The co-localization between high metal concentration and the crystal maps is yellow. The black arrows point out some deposits of $\mathrm{Fe}$ in the extracellular space. Each panel depicts an area of $450 \times 450 \mu \mathrm{m}$.

In addition to that, also crystal maps were compared with the distribution of the total fluorescence signal, with $\mathrm{Zn}, \mathrm{Fe}$ and $\mathrm{Cu}$ fluorescence since these metals can trigger in-vitro protein aggregation (Figure 3.7 left column). In this case, like in the other scans analyzed, there is almost no co-localization between the crystal maps and 
the areas with higher amounts of $\mathrm{Cu}$ and Fe. The highly ordered structures are in the extracellular regions as no overlap with $\mathrm{Zn}$-rich areas can be observed in Figure 3.7.

The most of the $\mathrm{Cu}$ and Fe appears to be concentrated in the intracellular space as it can be noticed in comparison to the $\mathrm{Zn}$ fluorescence, even if Fe has sometimes in some scans very bright spots due to the presence of extracellular depositions (see Figure 3.7 indicated with black arrow in the figure).

\subsubsection{Particle induced X-ray emission on PD patient tissue}

The XRF experiment conducted at ID13 was able to give interesting insights about the metal content in the intracellular and extracellular regions of the SN from PD patients. Although it would have been interesting to study LBs by the same technique, this was technically challenging. In fact, only one scanned region at ESRF contained a LB, while all other scans only contained NM positive cells without LB in the SN.

It is well known from previous studies that there is a metal dyshomeostasis with an increased amount of $\mathrm{Fe}$ in the PD patients' brains (particularly in the SN) (Szczerbowska-Boruchowska et al. 2012). In the literature is still unclear whether LBs are responsible for this dyshomeostasis or it's more a diffuse feature of the region.

For this reason, it was necessary to perform an elemental characterization at the single cell level. The idea was to compare the metal content of the LBs in NM positive cells with the same kind of cells without LBs from PD patients and Ctr samples.

To this, we took advantage of single cell resolution of the particle induced X-ray emission (PIXE) microscopy in combination with the conventional histology that allowed us to identify the LB distribution within the SN tissue. In total 24 samples were made from 4 different brains: 2 PD patients' brains and 2 control cases (multiple sclerosis) brains were used. 


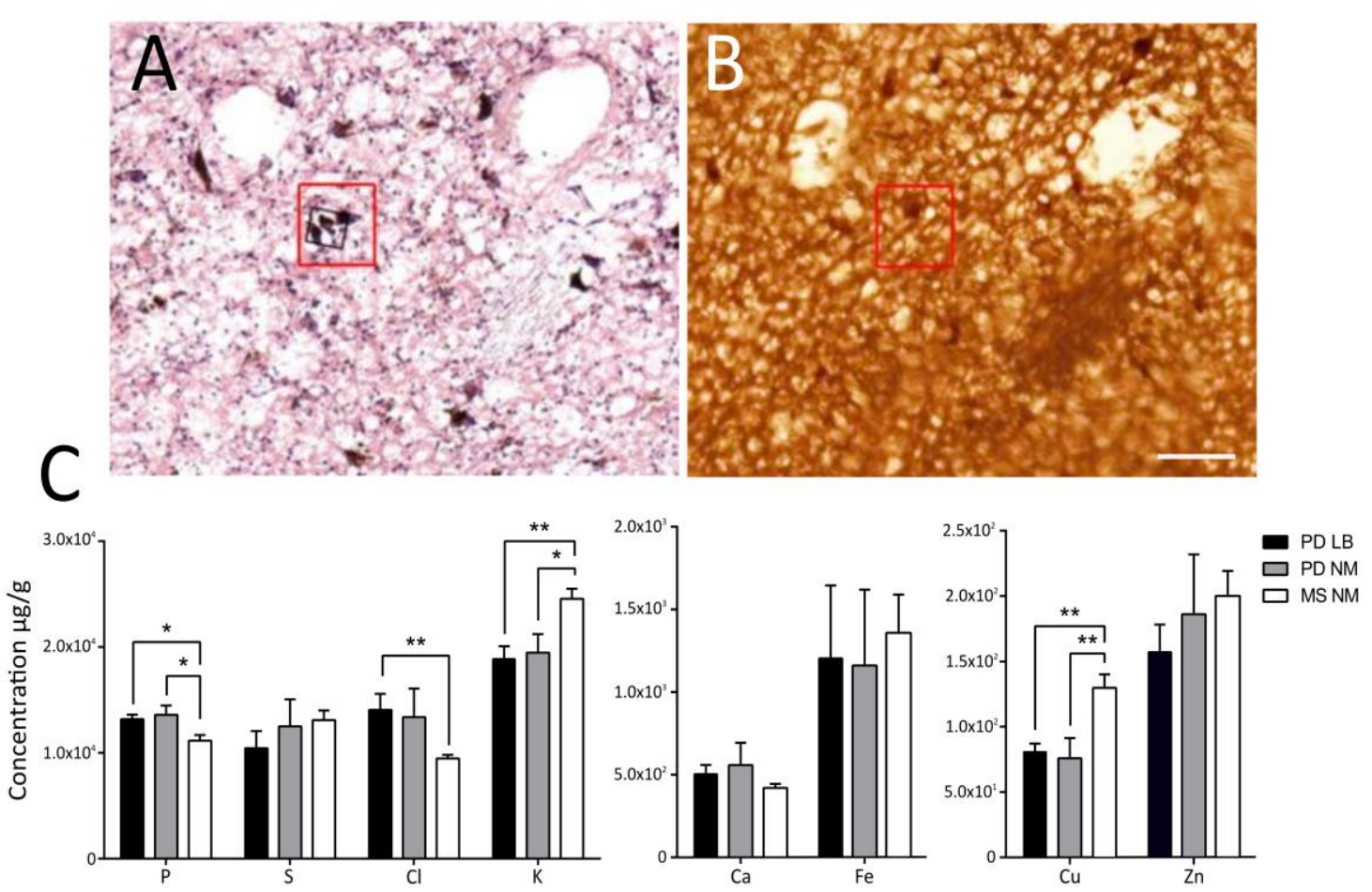

Figure 3.8 PIXE results and histology. A. H\&E histology of a section from a PD patient. The red square points out the presence of a $L B$ in the region. $B$. corresponding region showed in $A$. in the native section. The red square points out a probable cell with LB. Scale bar: $50 \mu \mathrm{m} \mathrm{C.} \mathrm{Absolute} \mathrm{elemental} \mathrm{quantification}$ from PIXE from PD samples. PD samples were screened for $L B$ s presence (LB PD) and analyzed in comparison to NM positive cells without $L B$ from the same section (NM PD) and NM positive cells from MS taken as controls (NM MS). Sections were taken from 2PD patients and $2 \mathrm{Ctr}$. Bars represent the mean \pm S.E.M., $n=4$ for PD LB; 5 for PD NM, 8 for MS NM. * $p$-value <0.05, ${ }^{* *} p$-value $<0.01$. $p$-values given with one-way ANOVA and Tukey test as post-hoc.

Exemplary of analyzed regions are shown Figure 3.8 A in which a LB was found (red square) with the Haematoxilyin and Eosin (H\&E) stain. LB in tissue sections after H\&E staining appear as rounded eosin-positive structures with often a peripheral lighter halo (Dunnett \& Björklund 1999; Spillantini et al. 1997). The picture in the panel $B$ shows the same region retrieved in the consecutive section that was kept native and it was analyzed by the means of PIXE.

The absolute concentration \pm S.E.M. of given elements for each kind of cells measured are reported in Figure 3.8C. The quantification of elements from LB from PD patient samples (LB PD) was compared with neighboring NM positive cells without LB (PD NM). As control NM positive cells from multiple sclerosis patients were used (MS NM).

The measured amount for P were: $13.15 \pm 0.63 \mathrm{mg} / \mathrm{g}$ for PD LB; $11.80 \pm 0.88$ $\mathrm{mg} / \mathrm{g}$ for PD NM and $11.00 \pm 0.48 \mathrm{mg} / \mathrm{g}$ for MS NM and the one-way ANOVA with Tukey test as post-hoc test, revealed that there is a significant difference between PD 
LB against NM MS and PD MN against NM MS ( $p$ value $=0.0297$ ). Regarding $\mathrm{Cl}$ the amounts were: $13.70 \pm 2.43 \mathrm{mg} / \mathrm{g}$ for PD LB; $12.90 \pm 2.24 \mathrm{mg} / \mathrm{g}$ for PD NM and $9.12 \pm$ $0.12 \mathrm{mg} / \mathrm{g}$ for MS NM and the same statistics showed a p-value 0.012 and a difference between PD LB and NM. The amounts of K showed a significant difference between PD LB against NM MS and PD MN against NM MS with a p-value of 0.0092 with the following concentrations: $16.94 \pm 1.55 \mathrm{mg} / \mathrm{g}$ for PD LB; $18.57 \pm 1.31 \mathrm{mg} / \mathrm{g}$ for PD NM and $23.60 \pm 1.11 \mathrm{mg} / \mathrm{g}$ for MS NM. Regarding metals, $\mathrm{Cu}$ is the only metal whose amounts show a significant decrease ( $p$-value 0.0035 ) in the samples of PD LB and PD NM compared with NM MS. The concentration measured were: $81.32 \pm 8.36 \mu \mathrm{g} / \mathrm{g}$ for PD LB; $77.34 \pm 8.55 \mu \mathrm{g} / \mathrm{g}$ for PD NM and $129.67 \pm 8.15 \mu \mathrm{g} / \mathrm{g}$ for MS NM. On the other hand, Fe values showed a huge variability in all the kind of cells measured since its concentrations were: $1.09 \pm 0.75 \mathrm{mg} / \mathrm{g}$ for PD LB; $1.17 \pm 0.76 \mathrm{mg} / \mathrm{g}$ for PD NM and $1.31 \pm 0.65 \mathrm{mg} / \mathrm{g}$ for MS NM.

\subsection{Alpha-synuclein and metal handling}

The previous section has taken into account the relationship that occurs between the structural and elemental features that are peculiar in samples coming from post mortem tissue of PD patients. Albeit these studies have the indisputable advantage to be as close as possible to the real pathology, they have also two main downsides: the first is that they depict only the end stage of the disease and not its development; the second is that there are many different external environmental factors that can influence disease progression individually in each patient.

Cellular models are thus, under certain aspects, very useful to model pathophysiology in a defined environment. In fact, they are usually easy to follow over time and the conditions, in which the experiment is carried out, can be tightly controlled.

Therefore we took advantage of cultures of primary midbrain neurons (PMNs) transfected with AAV overexpressing aSyn in order to determine whether aSyn has an effect on metal distribution within neuronal cells as it happens in the SN of PD patients. 


\subsubsection{Alpha synuclein overexpression and Fe supplementation in} primary neuronal cultures

At this point, we wanted to investigate if aSyn could be responsible for the metal dys-homeostasis that our data from the previous sections and a solid consensus in the literature pointed out as a very important step in the pathogenesis of PD. The interaction between aSyn and metals has been known for a long time and it has been extensively studied in cell free systems, but those system cannot take into account the role played by other proteins present in the cellular milieu.

To this, we took advantage once again of the PIXE for analyzing PMNs cells transfected with AAV expressing aSyn and EGFP or EFGP only as control. In addition to that, since the amount of Fe is higher in the PD patients compared to Ctr, cells were supplied with $50 \mu \mathrm{m}$ of $\mathrm{Fe}^{+2}$ for 3 hours.

The resulting quantification is reported in Figure 3.9. In panel A, two experiments are shown together. In absence of Fe supply $\mathrm{Cu}$ emerged as the only metal that was significantly altered in concentration. In fact, cells overexpressing aSyn had $47.54 \pm 5.46 \mu \mathrm{g} / \mathrm{g}$ of $\mathrm{Cu}$ whereas the cells expressing EGFP only had $28.61 \pm$ $1.03 \mu \mathrm{g} / \mathrm{g}$.

When the PMNs were supplemented with $\mathrm{Fe}^{+2}$ for 3 hours, the concentration of $\mathrm{Cu}$ still increased slightly, even if not significantly $(38.51 \pm 3.34 \mu \mathrm{g} / \mathrm{g}$ for aSyn transfected cells and $34.08 \pm 2.23 \mu \mathrm{g} / \mathrm{g}$ for Ctr cells).

Regarding Fe concentration, the cells expressing aSyn contained $717.89 \pm$ $130.24 \mu \mathrm{g} / \mathrm{g}$ compared to $390.75 \pm 56.68 \mu \mathrm{g} / \mathrm{g}$ in the EGFP transfected cells, at the same conditions. The concentration of $\mathrm{Ca}$ in the intracellular space for cells expressing aSyn was $4.34 \pm 1.27 \mathrm{mg} / \mathrm{g}$ and $1.88 \pm 0.94 \mathrm{mg} / \mathrm{g}$ for the Ctr cells.

Studying the elemental distribution maps, it was clear that there was a difference in the total fluorescence intensity between the nucleus (in the middle of the cell) and the cytoplasm (Figure 3.9). This can be due to the elemental concentration per se or the fact that XRF results in a sum spectrum of all the atoms encountered, which would suggest higher concentrations depending on sample 
thickness. Therefore the correction given by the Rutherford Backscattering (RBS in the figure) is crucial to calculate the correct amounts of elements corrected for the thickness, allowing an absolute concentration of each element analyzed. The cell shown in this figure was transfected with aSyn and exposed to $\mathrm{Fe}^{+2}$ for 4 hours. The Fe map indicates a high concentration of $\mathrm{Fe}$ inside the cell compared to the surroundings (in line with the quantification in panel A). The concentration of $\mathrm{Cu}$ is very small and not much different to what can be found in the extracellular space. The $\mathrm{Zn}$ distributions is in agreement with the morphology of a regular cell, displaying a higher concentration due to the fact that NM positive cells are rich in this element(Zecca et al. 1996). 


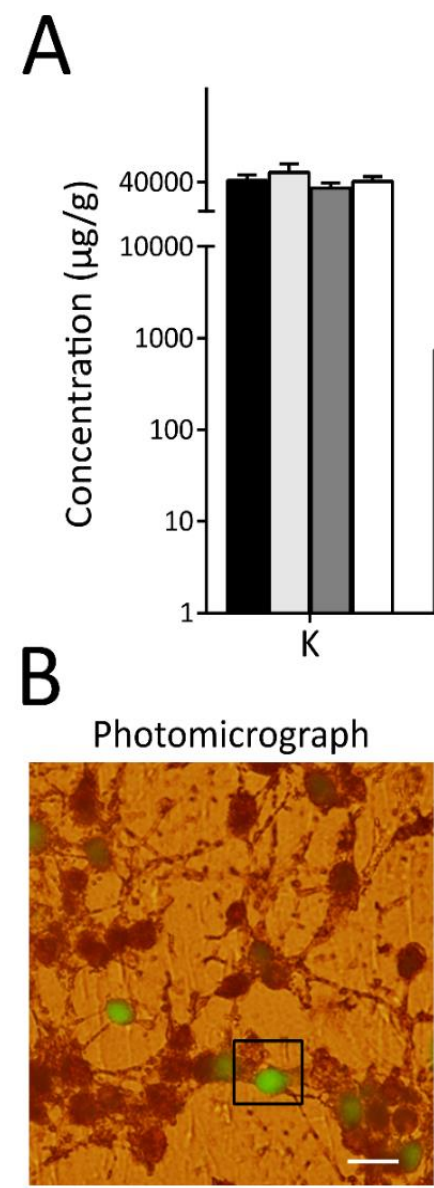

Fe map

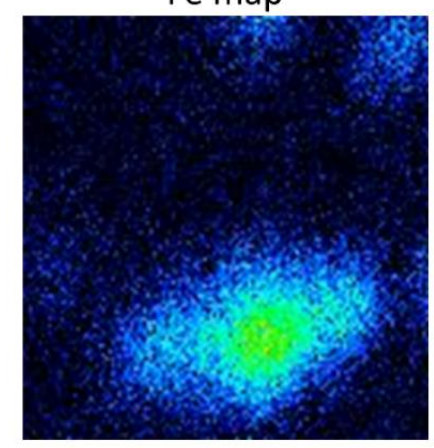

Total PIXE

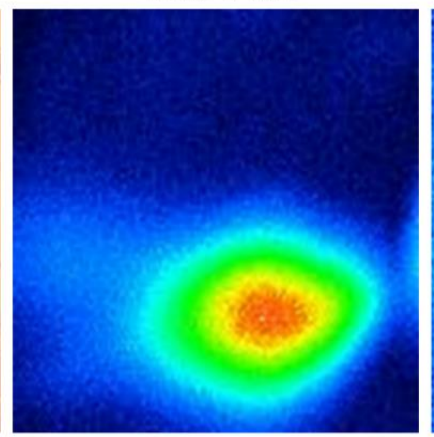

Cu map

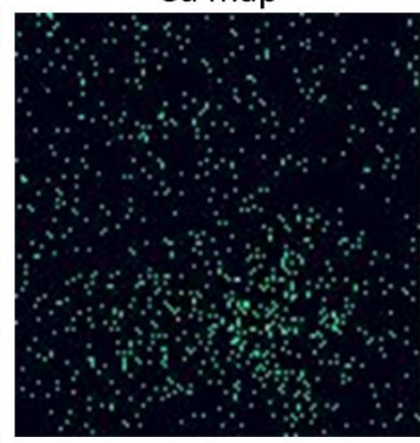

EGFP

$\square$ aSyn

$\square \mathrm{EGFP}+\mathrm{Fe}$

$\square \alpha$ Syn + Fe

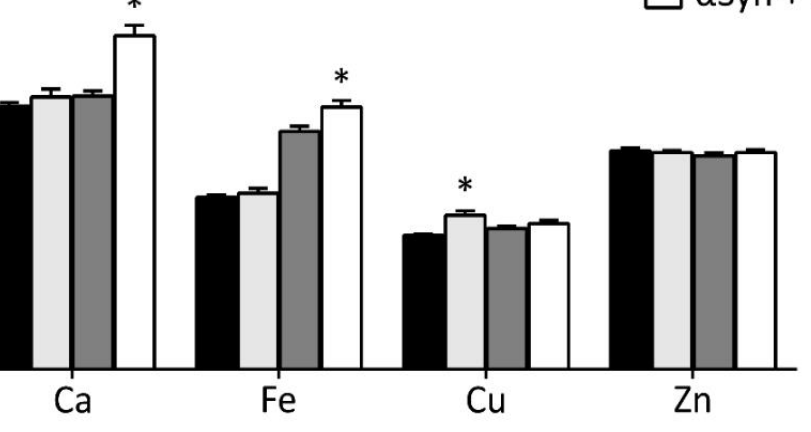

RBS

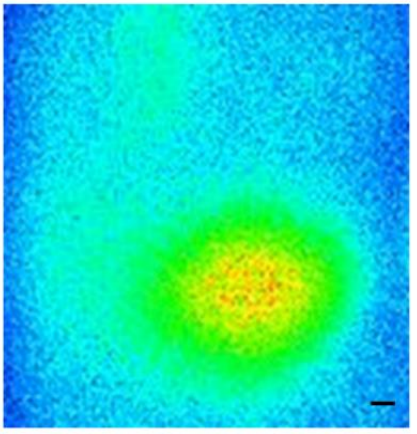

Zn map

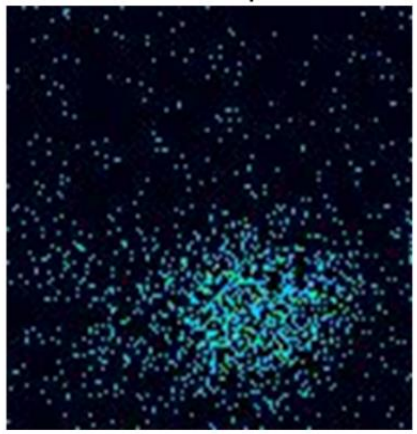

Figure 3.9 PIXE measurements of primary midbrain neurons overexpressing aSyn with or without Fe supply. A. Elemental quantification of the PMNs transfected with AAV.EGFP.ASYN. B. Exemplary pictures of elemental distribution maps of a neuronal cell transfected with aSyn after Fe exposure. The photomicrograph shows the presence of green cells that have been transfected with aSyn. The square points out the single cells that was analyzed by PIXE. Scale bar: $20 \mu \mathrm{m}$. The same cell evaluated for Total events (Total PIXE); Rutherford back scattering (RBS) and the elemental map of $\mathrm{Fe}$, Cu and $\mathrm{Zn}$ are shown. Scale bar: $1 \mu \mathrm{m}$. 


\subsubsection{Alpha-synuclein overexpression and metal transporting proteins}

From our previous sets of experiments, it can be stated that aSyn has indeed an impact on the metal concentration and distribution within the cell. This raised the question how aSyn can exert this effect. Several previous reports suggest a direct binding between aSyn and metals. This does not exclude that the binding could be combined with a possible influence of aSyn on the regulation of metal transporting proteins.

To answer to this question, six metal transport proteins were selected to be studied from a transcriptional point of view (mRNA levels) and from a protein point of view. The selected proteins are crucial for $\mathrm{Cu}$ and Fe trafficking as these two metals are the most affected by aSyn overexpression. For $\mathrm{Cu}$ metabolism the proteins chosen were: CTR1 that enables $\mathrm{Cu}$ import inside the cell; ATP7a and APT7b are two isoforms of the most important $\mathrm{Cu}$ exporting proteins. For Fe metabolism the trafficking proteins chosen were: DMT1 and TfR for the import of Fe; the last protein taken into account is Fpn1 that can export Fe from the cell.

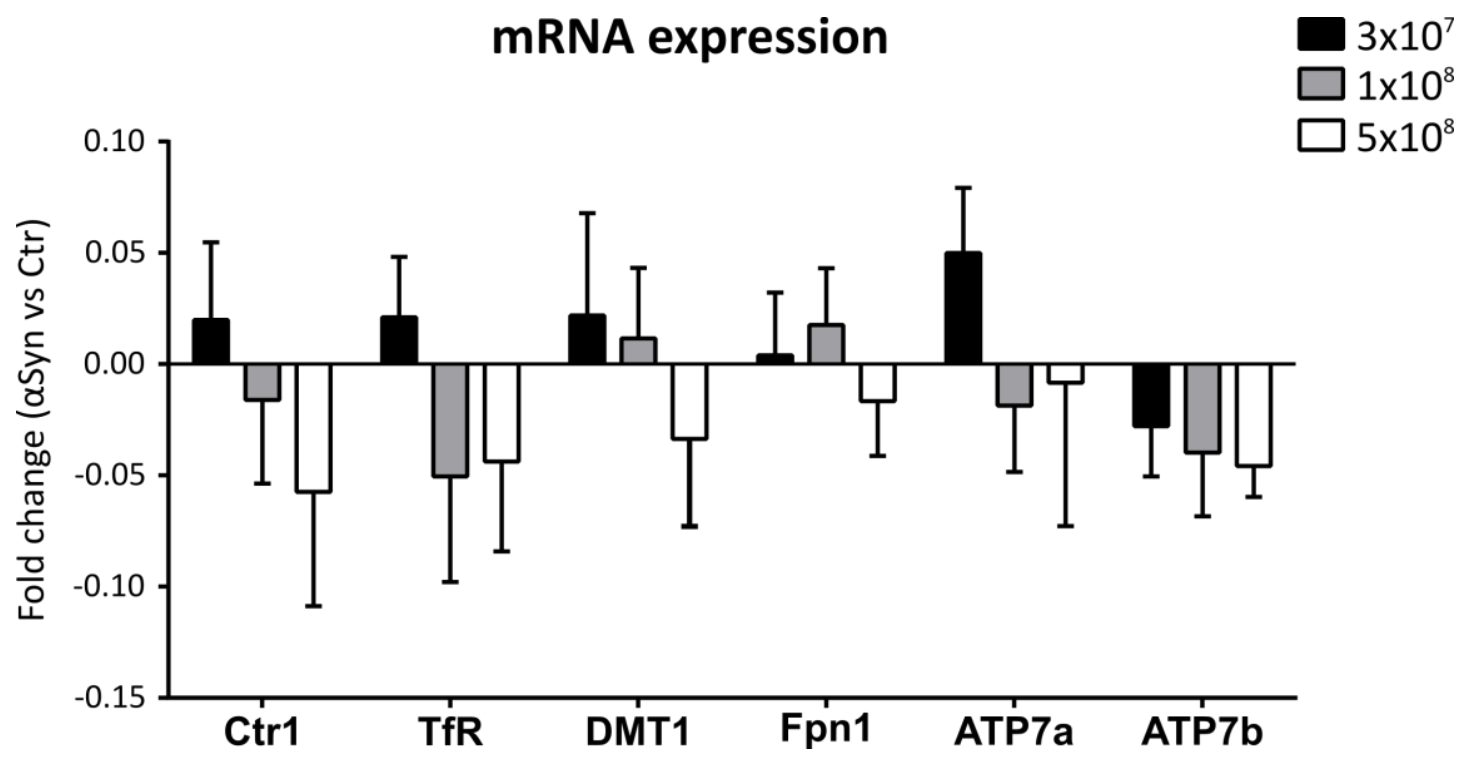

Figure 3.10 Evaluations of metal transport proteins levels influenced by increasing concentration of aSyn viral vectors. Quantification of the expression of $m R N A$ of the 6 most abundant proteins for metal trafficking: ATP7a, ATP7b, CTR1, DMT1, Fpn1 and TfR. No statistical differences with Student's t-test. $n=4$ for each protein. 
The results of this project are summarized in Figure 3.10 and Figure 3.11.The levels of the mRNA of the aforementioned proteins are evaluated in presence of increasing amounts of adeno-associated virus (AAV) with or without aSyn overexpression ( $n=4$ independent cultures). The viral amounts per well tested were: $3 \times 10^{7} ; 1 \times 10^{8}$ and $5 \times 10^{8}$ for both the viruses tested. The results (see Figure 3.10 ) are expressed as $\Delta \Delta C$ t between aSyn transfected cells and Ctr transfected cells (see chapter 2). The change in the expression of the mRNA of the proteins is very mild and there are no significant differences. There is only a slight effect for the CTR1 protein that is down regulated as the concentration of aSyn increases.

At the protein level, the WBs for the metal-handling proteins show that there are no great differences despite the fact that PMNs were overexpressing increasing amounts of aSyn (Figure 3.11). In fact, in the band intensity ration of the proteins compared to GAPDH of 4 independent cultures states that the level of those proteins remains unchanged. The only protein that appears to be regulated is CTR1 whose levels are inversely proportional to aSyn levels in a significant way $(p=0.045)$. 

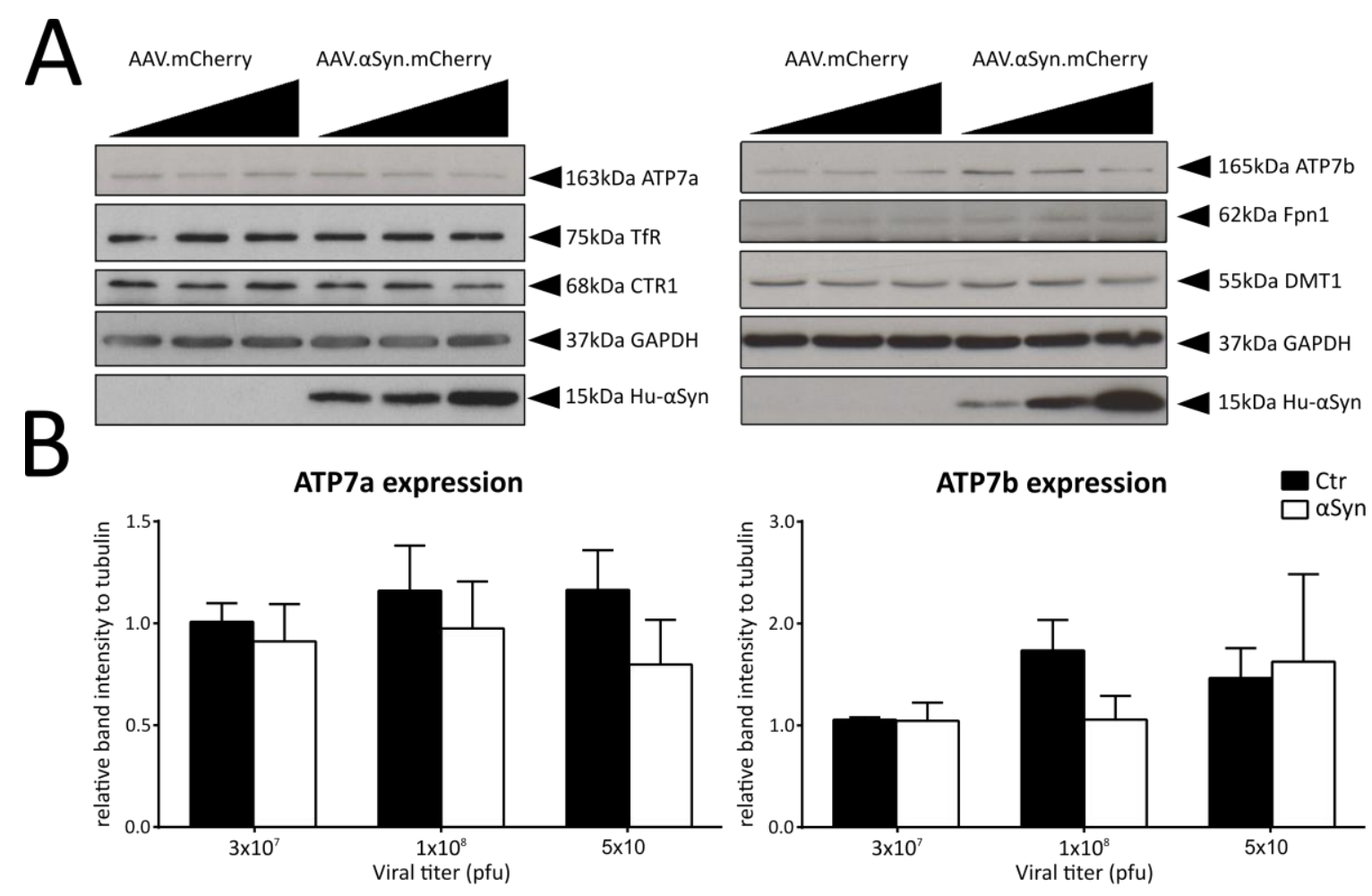

TfR expression

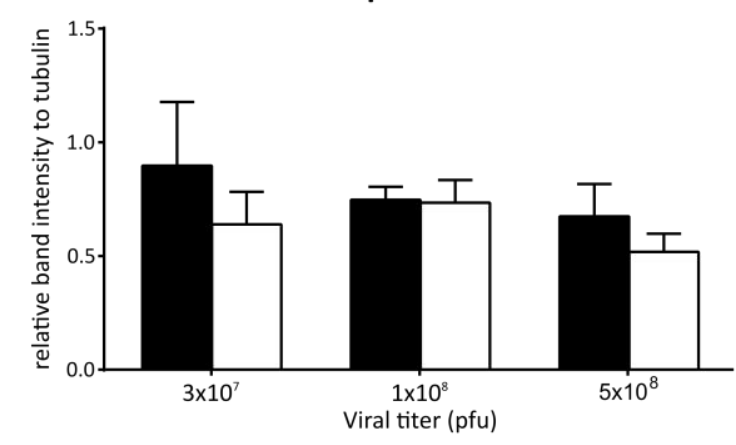

Fpn1 expression

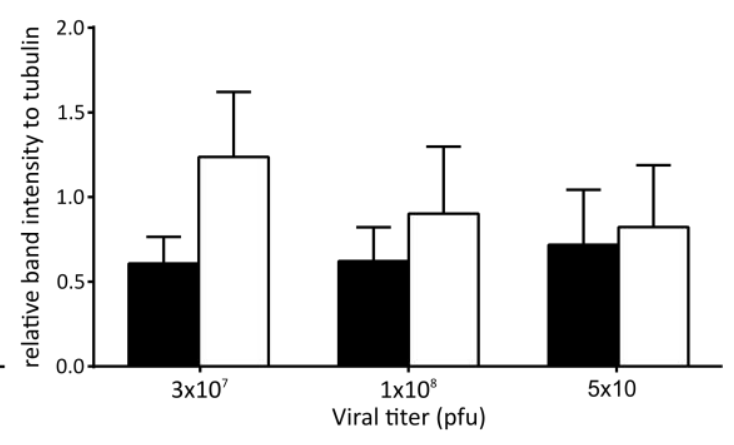

CTR1 expression
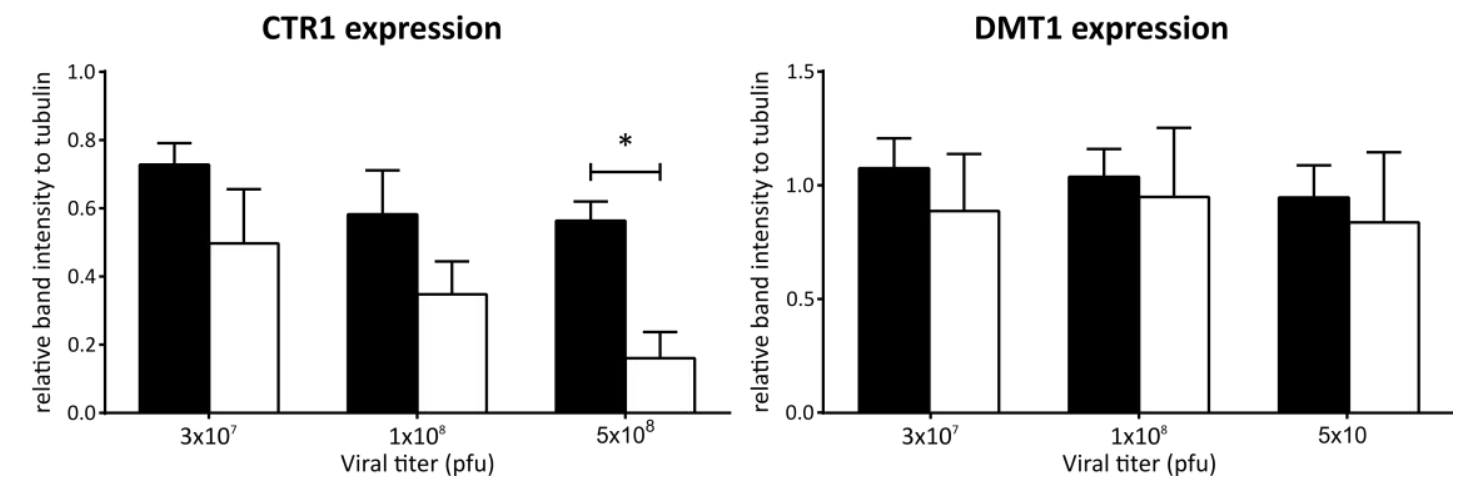

Figure 3.11. Evaluations of metal transport proteins levels influenced by increasing concentration of aSyn viral vectors. A. Exemplary images of WBs for all the metal trafficking proteins tested. $B$. Quantification of the expression of the 6 most abundant proteins for metal trafficking: ATP7a, ATP7b, CTR1, DMT1, Fpn1 and TfR. CTR1 is significantly down regulated by aSyn. Student's $t$-test. $n=4$ for each protein. ${ }^{*} p$-value $<0.05 ; n=4$ independent cultures. 


\subsection{In-vivo aggregation study: A53T mice and Fe supply}

The data presented so far has been obtained in two systems: the post mortem tissue from PD patients and cell cultures. The post mortem tissue provides very useful insights in the disease pathogenesis itself as it originates from real PD patients. But these samples are often inhomogeneous as the etiopathology of PD is multifactorial. Each patient has different environmental factors and it's usually very complicated to find unifying traits with the limited amount of material available. On the other hand, cell cultures are very valuable tools for the biochemical characterization of disease mechanisms, but they lack the complexity of an organism meant as a whole.

For those reasons, we decided to take advantage of an in vivo mouse model to study whether aSyn aggregation could be affected by Fe supply.

To study this interaction, we used the model of the transgenic murine line called B6;C3-Tg(Prnp-SNCA*A53T)83Vle/J (short: A53T mice) in combination with an Fe intoxication protocol ( $40 \mathrm{mg} / \mathrm{kg}$ bw/day given between $P 10$ and $P$ 17). The A53T TG animals were divided in 4 groups: Ctr, Fe, DFPctr and DFPFe (see Chapter 2) and then different behavioral and biochemical evaluations were carried out.

\subsubsection{Behavioral analysis: rotarod}

In order to assess the effect of interaction between aggregating aSyn and Fe on coordination, balance and motoric learning skills, the animals were tested on the rotarod.

The time frame of this experiment is shown in Figure 3.12A. After birth from $P$ 10 to $P 17$ the animals were either given oral gavage with $40 \mathrm{mg} / \mathrm{kg}$ bw/day of Fecarbonyl or vehicle only. From P 100 till sacrifice mice drunk either normal water or water supplemented with the Fe chelator DFP. The rotarod assessment was performed once a week from P 220 until sacrifice.

The performance of the various groups in the learning phase can be seen in Figure 3.12B 
A

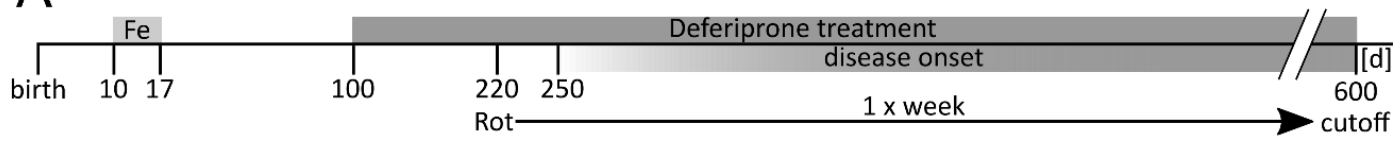

B Rotarod performances

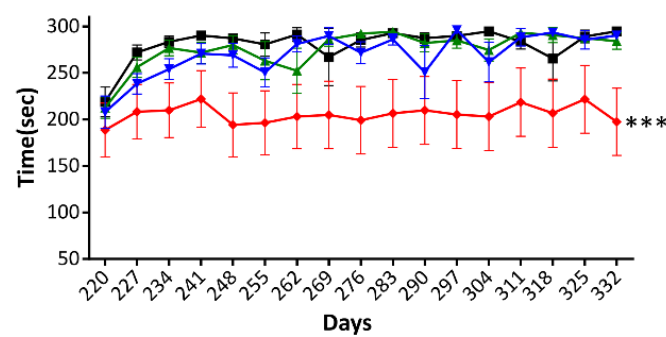

C Rotarod performances before sacrifice

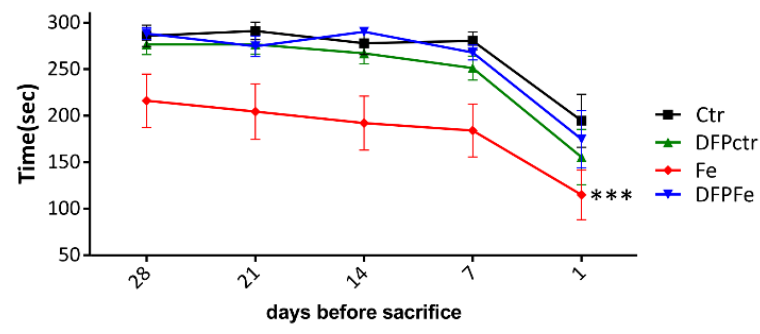

Figure 3.12. Rotarod evaluation of A53T mice. A53T TG mice were fed with $40 \mathrm{mg} / \mathrm{kg}$ bw iron carbonyl for 7 consecutive days (from $P 10$ to $P$ 17). At $P 100$ animals were supplied with $10 \mathrm{mg} / \mathrm{kg}$ bw of the Fe chelator DFP. Rotarod was performed starting from $P 220$ weekly. B. Rotarod performances of the learning phase. The Fe fed animals fail to learn how to complete the task as they never reach the 300 seconds. DFP has no effect of this task as the DFP fed animals perform as good as the Ctr animals. The DFP is able to rescue the toxic effect of Fe as the DFPFe animals perform as good as the Ctr animals. ANOVA with repeated measures $p$-value <1*10-6 C. Rotarod performance before sacrifice. The trend between different treatments is kept stable like in the learning phase until the day of sacrifice. ANOVA with repeated measures and Tukey as post hoc-test $p$-value $<1 * 10-4$.

The Ctr animals learn to accomplish the task within the first 4 or 5 repetitions on the rotarod and then they keep their performance being able to run in the average $280 \pm 9 \mathrm{sec}$ on the rotarod. This is also true for the animals of the DFPctr group that have been drinking water with DFP ( $275 \pm 10 \mathrm{sec})$. Conversely the animals that have been treated with Fe display a learning curve isn't as steep as the other groups and they never manage to learn how to stay on the rotating rod for 300 seconds, in fact their time on the rotarod is in average of $205 \pm 30 \mathrm{sec}$. Strikingly the group of DFPFe learns how to perform rotarod just as good as the untreated animals (270 $\pm 11 \mathrm{sec}$ ) and they keep their performances for a long time. ANOVA for repeated measures shows a $p$-value $=1 \times 10^{-6}$ for the difference between Fe treated animals and the other groups.

In the panel $\mathrm{C}$ of the same figure, the last month (5 weekly performances) before sacrifice are shown. The differences that were noticed during the learning phase are still present. In fact, with the exception of the last day of testing (disease onset), the animals still perform in the same way: Ctr $278 \pm 7$ sec; DFPctr $266 \pm 11$ sec; Fe $197 \pm 27 \mathrm{sec}$ and DFPFe $282 \pm 11 \mathrm{sec}$. The ANOVA for repeated measures shows a $p$-value $=1 \times 10^{-4}$ for the difference between Fe treated animals and the other 
groups in this case as well. On the very day of disease onset (last point in the graph of panel C) all the groups perform quite the same and the treatment effects are not distinguishable anymore.

\subsubsection{Behavioral analysis: Novel object recognition (NOR)}

Another behavioral test that was performed on the different groups of transgenic animals is the novel object recognition (NOR) test. NOR allows the evaluation of the general cognitive abilities in animals which are naturally curios. Therefore they should spend more time in exploring new objects rather than familiar ones. This test can also be seen as a test for a qualitative determination of the memory of the mice.

The time frame of this experiment (Figure 3.13 A) is similar to the time frame of the rotarod. The animals are tested here at P 250 and when they show the signs of the disease onset (weight loss $\geq 20 \%$ in a week and rotarod failure).

An exemplary picture of the test evaluation is reported in the panel B of Figure 3.13. A single mouse is introduced in an arena with one familiar object $(F)$ and a novel object (N). The software Ethovision XT 8.5 calculates automatically the time spent in the zone of interest of the different objects (Z. o. I.) as well as the distance run during the test.

The results of the performances at $P 250$ ( $n=5$ animals per group) are presented in Figure $3.13 \mathrm{C}$. At this time point, all the animals are more interested in the novel object rather than in the old one (as the time spent with the novel object is always more than $50 \%)$.

The A53T TG Ctr and the DPFctr mice spend almost the $75 \%$ of their time exploring the new object, showing to be capable to differentiate between the object and remembering what is old and what is new. This doesn't seem to be the case for the animals treated with Fe only. They still spent slightly more time with the new object ( $54 \%$ of the total time) but the amount of this time is less that the time spent by the Ctr animals. In this case, there is a tendency the treatment with DFP helps the 
mice that received Fe (DFPFe) in improving their performances. In fact those animals are almost exclusively interested in the new object ( $78 \%$ of total time).

All the tested treatment groups run more or less the same distance (Figure 3.13 D). When the mice are tested at disease onset, the differences seen at P 250 tend to level out (Figure $3.13 \mathrm{E}$ ) as all the mice are similarly interested in the new object (about $65 \%$ of total time for all groups).Also in this case, there is no difference in the distance run in the 5 minutes of the test (Figure $3.13 \mathrm{~F}$ ). 


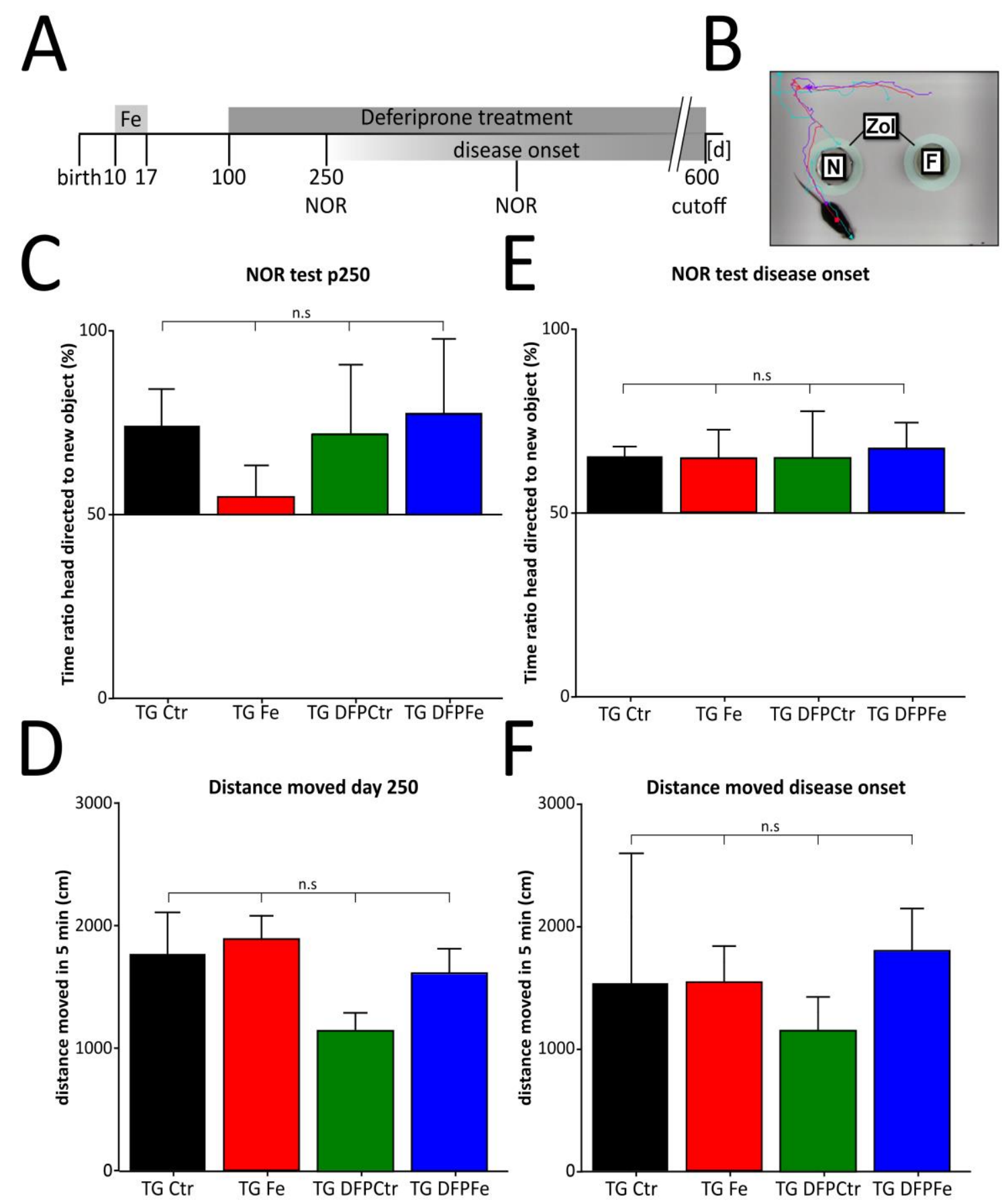

Figure 3.13. Novel object recognition test. A. A53T TG mice were fed with $40 \mathrm{mg} / \mathrm{kg}$ bw iron carbonyl for 7 consecutive days (from $P 10$ to $P$ 17), at $P 100$ animals were supplied with $10 \mathrm{mg} / \mathrm{kg}$ bw of the Fe chelator DFP. NOR performed at $P 250$ and disease onset. B. mice were put in an arena with a familiar $(F)$ and a novel object (N). The time spent heading towards the zone of interaction (ZOI) with the objects was analyzed together with general activity. C. NOR test performed at P 250. The Fe treated animals perform worse than the Ctr animals and have more problems in discriminating the novel object while the treatment with DFP appears to be beneficial for this task. D. the distance run during the test at $P$ 250. E. There were no more differences at disease onset stage for NOR. F. distance run during the test at disease onset. ANOVA test. ( $n=4$ for TG Ctr, $n=5$ for the other groups at $P 250 ; n=4$ for TG Ctr, $n=5$ for TG DPFctr, $n=8$ for TG Fe and TG DFPFe at disease onset). 


\subsubsection{Behavioral analysis: Catwalk}

The last motor evaluation performed on A53T TG animals was the Catwalk analysis. The Catwalk is an apparatus for automatic motor evaluation of the gait of rodents that are freely moving inside a walkway. No training is required for mice and there is no task to be learned.

The timeframe of this evaluation is very similar to the NOR. As can be seen in Figure $3.14 \mathrm{~A}$, the mice were fed with $40 \mathrm{mg} / \mathrm{kg}$ bw/day of Fe-carbonyl (or vehicle) between $P 10$ and 17 and started the DFP treatment $(10 \mathrm{mg} / \mathrm{kg} \mathrm{bw} / \mathrm{day})$ in the drinking water from P 100 on. They underwent the Catwalk analysis at P 250 and at disease onset.

The Catwalk is able to assess several important gait parameters in a quantitative automatic manner, such as stand, swing, step cycle, stride length, print length, print width and print area (Figure 3.14B).

The panel $\mathrm{C}$ of the figure is a comparison of the performances of the TG animals at disease onset with the littermate WT Ctr. The selected parameters for comparison were: run average speed mean, max contact area and stride length. These parameters confirm that there are significant differences between the WT Ctr and the TG animals at the day of disease onset, albeit these differences are not significant among the TG animals, independently from treatments received.

At the time point of sacrifice, no significant difference in weight was observed between the groups (Figure 3.14D).

In contrast to that, when only the TG mice are considered, there is a tendency for some parameters that are tightly linked with the weight (such as the "max intensity" as reported in Figure 3.14F) to be worse in all the four paws in the heavier animals thus providing a hint of how the $\mathrm{Fe}$ is exacerbating the disease model. Overall, however the effect of Fe intoxication is very mild and at P 250 there are no difference among the groups. 

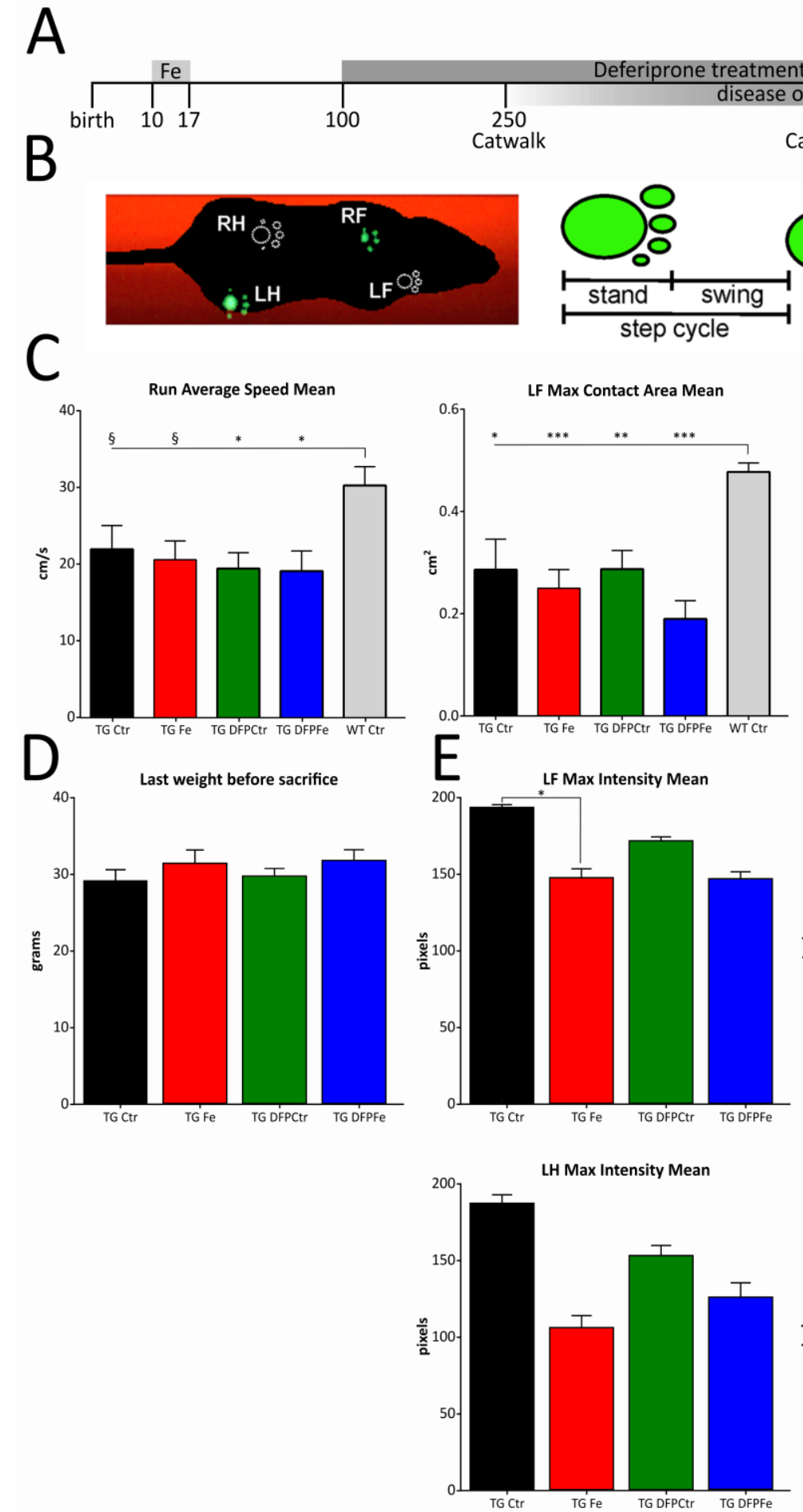

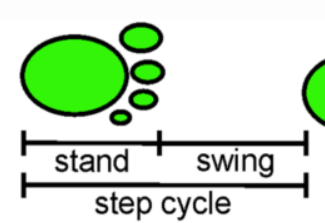

600
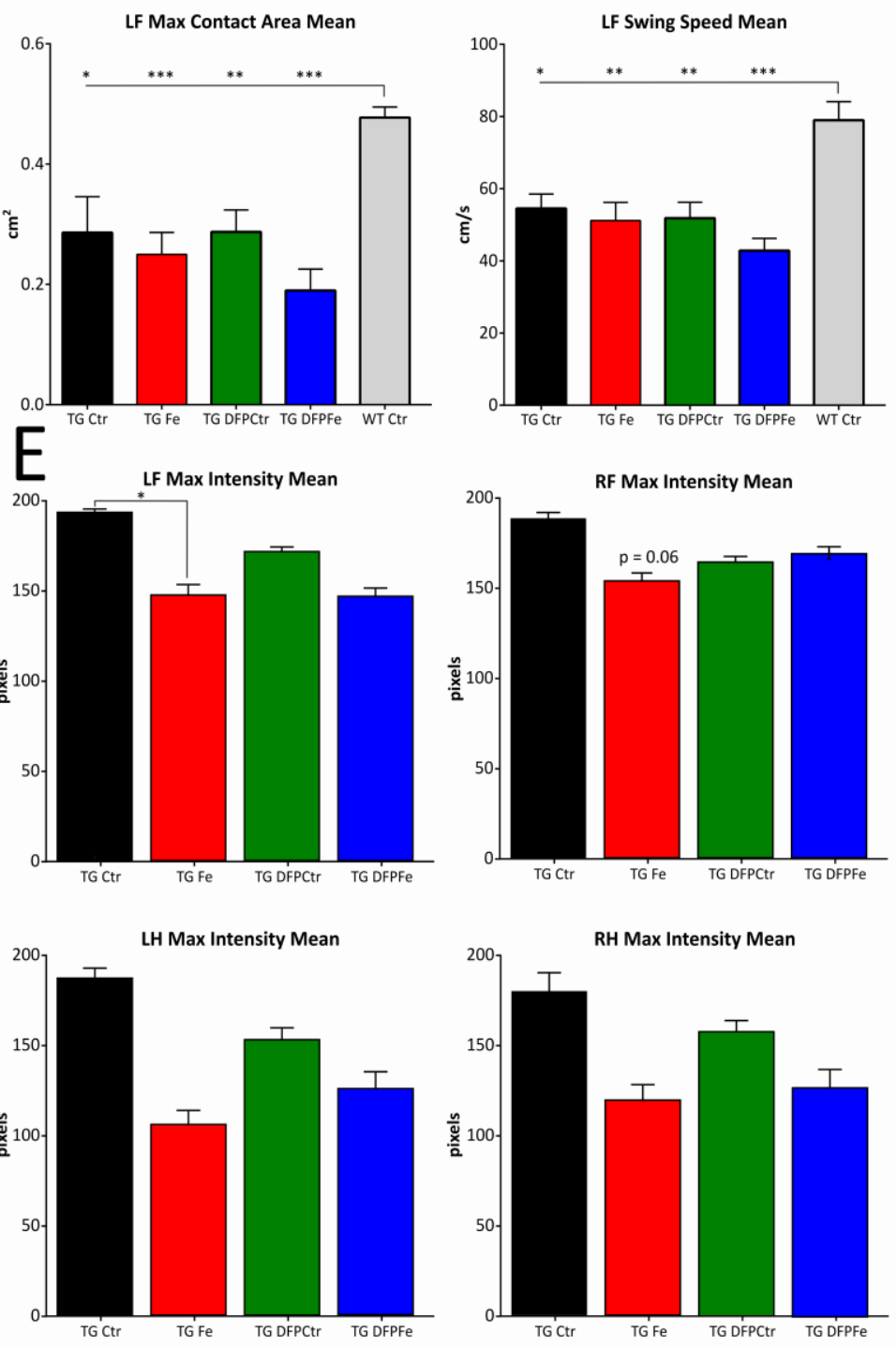

Figure 3.14. Catwalk results. A53T TG mice were fed with $40 \mathrm{mg} / \mathrm{kg}$ bw for 7 consecutive days (from p10 to p17), at $P 100$ animals were supplied with $10 \mathrm{mg} / \mathrm{kg}$ bw of the Fe chelator DFP. Catwalk at $P 250$ and disease onset. B. Noldus Catwalk XT gait detection system of the mice footprints of mice (RF = right front, $L F=$ left front, $R H=$ right hind, $L H=$ left hind). $C$. At day of disease onset different gait parameters like run average speed mean, max contact area and, swing speed were compared with the WT to confirm that the model is reproducible to what is known in literature. D. Comparison of last weight before sacrifice. Fe treated animals are slightly heavier than the untreated animals. E. Comparison of Max intensity of the 4 paws as exemplary gait parameter in a comparison of A53T TG mice only. ANOVA and Tukey test as post hoc. ${ }^{*} p$-value $<0.05 ;{ }^{* *}$-value $<0.01 ;{ }^{* *} p$-value $<0.001 ; n=4$ for TG Ctr, $n=5$ for TG DPFctr, $n=8$ for TG Fe and TG DFPFe, $n=10$ WT Ctr. 


\subsubsection{Analysis of survival}

When the TG animals showed signs of the disease (weight loss $\geq 20 \%$ within one week and rotarod failure), this time was taken as end point, and the animal were sacrificed. The Kaplan-Meier survival curve was calculated on those time points.

The mean survival for each group was: $379.43 \pm 30.25$ days for the $\mathrm{Ctr} ; 401.13$ \pm 32.45 days for the Fe group; $398.23 \pm 32.71$ days for the group that were receiving only DFP; and $394.47 \pm 40.10$ days for the DFPFe. The average survival was $395.05 \pm$ 17.02 days. The calculated statistic $p$ value was of 0.989 . There are no statistical differences among the groups. No WT mice were taken into account as they don't develop the disease.

\section{Kaplan-Meier survival curve}

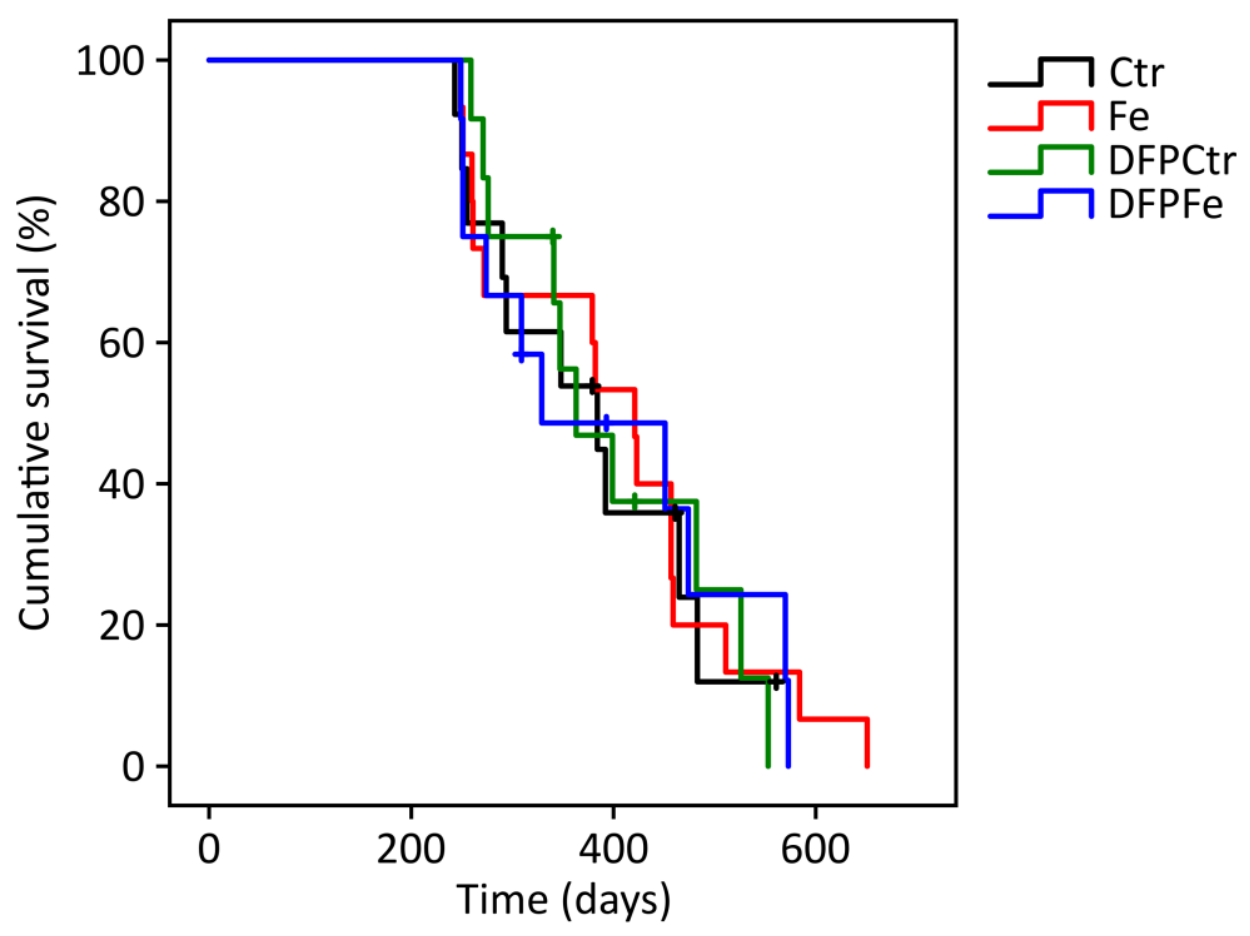

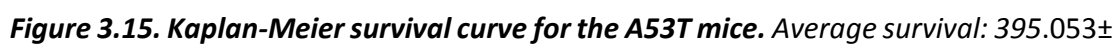
17.016 days. $p$-value $=0.989$. ( $n=12$ for all the groups). 


\subsubsection{Biochemical characterization of the aggregation}

In order to further characterize the interaction that occurs between Fe and aSyn in our model, we performed dot blots for assessing the amount of aggregated aSyn in TG mice brains. WT mice didn't show any aggregated form of aSyn after digestion. Seven brain regions were dissected for analysis: olfactory bulb, cortex, hippocampus, pons and medulla, midbrain, striatum and cerebellum. Since there is evidence in the literature that most of the aSyn aggregates A53T murine model are found in the cortex (Giasson et al. 2002), this area was selected to be analyzed with dot blots after proteinase $\mathrm{K}(\mathrm{pK})$ digestion.

The quantification (Figure 3.16 bottom) was carried out with 5 animals per group of all 4 different groups (Ctr, DFPCtr, Fe and DFPFe). The one-way ANOVA showed that there are not statistically significant differences ( $p$-value: 0.08 ) but there are clear tendencies. Fe-treated animals show a trend for a higher amount of aggregated synuclein ( $128 \pm 25 \%$ of the Ctr). A similar value can be seen also in the DFPFe group with a dot blot intensity of $127 \pm 29 \%$ of the Ctr. Interestingly the CtrDFP animals have less aggregated aSyn, and the mean intensity of the band is $48 \pm 15 \%$ of the Ctr. 

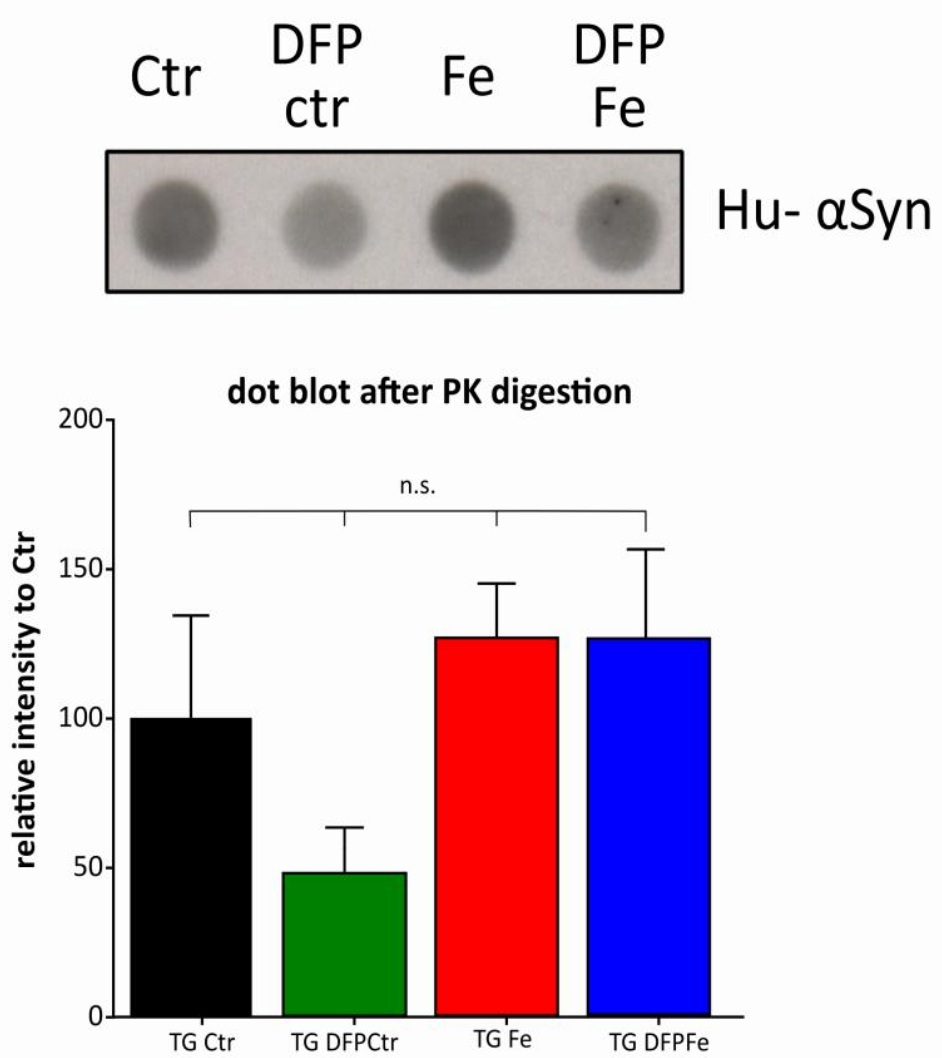

Figure 3.16 Dot blot assay for aggregated human aSyn in the cortex of A53T mice. The mouse brains were homogenized and digested with PK for $10 \mathrm{~min}$. Top. Exemplary film of dot blot. Samples were blotted on spots and revealed through an anti-HuaSyn antibody. Bottom. Quantification of spot intensities in the dot blot. ANOVA showed no statistical differences. $n=5$ mice per group 


\section{Discussion}

Parkinson's disease (PD) is a multifaceted, multifactorial disease. A diagnosis is possible only al late stage of the disease, when most of the dopaminergic neurons and their projections are already gone and the aggregation processes of aSyn is already in an advanced state so that the motor symptoms become evident (Braak et al. 2003).

There are, at present, no curative treatments for PD as the causes of the disease are still elusive. Several mechanisms have been proposed to be involved in PD, like failures in protein recycling, protein aggregation, mitochondrial dysfunctions, increased oxidative stress, excitotoxicity and metal toxicity, but it's still unknown whether some of these mechanisms can have a protective or pathogenic effect (Galvan \& Wichmann 2008; Dexter \& Jenner 2013).

\subsection{Alpha-synuclein aggregation and X-ray imaging}

Oligomers of aSyn are believed to be the most toxic species contributing to the pathogenesis of PD (Conway et al., 2000). The oligomers represent a heterogeneous and poorly characterized group of structural conformers that may share common features such as the mechanisms of actions or aggregation pathways. The toxicity of the oligomers may impact cells in different manners, like the disruption of membranes, mitochondrial depolarization, cytoskeleton changes, impairment of protein clearance pathways, and enhanced oxidative stress (Roberts \& Brown, 2015).

aSyn oligomers are difficult to structurally characterize in a cellular environment. Studies carried out so far, have been using purified protein that was left for incubation in different buffer conditions and for different amounts of time. These analyses pointed out the presence of numerous instable pre-fibrillar species on their way to aggregation. The techniques applied were mostly atomic force microscopy, Raman spectroscopy and size exclusion chromatography (Chen et al. 2015; Apetri et al. 2006).

In our experimental approach, we used diffraction imaging to characterize the aggregation states of proteins in PD models and human tissue. 
To that, two different systems were analyzed: the first was a cellular model in which aggregates appear as a consequence of transfection of two plasmids; the second system were tissue sections coming from a PD patient and a Ctr.

The characterization was done by the means of scanning transition X-ray microscopy and X-ray diffraction in the SAXS and WAXS range.

\subsubsection{Aggregates in $\mathrm{H} 4$ cells do not give a signal in STXM}

To evaluate the diffraction signal coming from pre-aggregated, e.g. oligomeric, forms of aSyn in a cellular model, we took advantage of the aggregates that can be formed in a in a neuroglioma cell line that was analyzed by scanning transmission Xray imaging (SXTM). The aggregates are formed through the overexpression of SynT (a chimeric protein of aSyn fused with the first 100 aminoacids of EGFP) and Synphilin-1 (a protein that interacts with aSyn and co-localizes with LB) (McLean et al. 2001).

Our results point out that there is no clear signal in the dark-field of the SXTM arising from the SynT/Synphilin-1 aggregates that were visible through immunostaining. The lack of scattering is likely due to the absence of highly ordered structures in these aggregates. In contrast, other structures like the nuclei of cells in the condensation phase of apoptosis, gave a very bright signal. In fact in the condensation phase, the DNA in the cells undergoes condensation and this results in a higher electron-density that was detected by STXM (Makrigiannakis et al. 1999).

However, even if there was no signal coming from aSyn aggregates, it's important to remark that the presence of antibodies that were surrounding the aggregates might have had an impact on the signal arising from these structures and could possibly distort it. On the other hand, it is technically not possible to detect the SynT/ Synphilin-1 aggregates without immuno-staining because only few $\mathrm{H} 4$ cells get properly transfected ( $10 \%)$ and about only half of the transfected cells develop aggregates (McLean et al. 2001). Hence, these intrinsic characteristics of the model, do not combine well with the resolution at single cell level of the STXM. 
A possible approach to avoid signal artifacts due to antibody presence, would have been scanning wide areas of the holder with several cells looking for a signal in the dark-field of the STXM. After acquisition of the scan, cells could have been stained with antibodies comparing the localization of the signals in the dark-field with the localization of the immuno-stained aggregates. This approach exploits the fact that the radiation damage in cells after STXM operating in the soft X-ray range is mild (Gianoncelli et al. 2015; Bernhardt et al. 2016), and it's very likely that the antibodies could find their target structures even after being irradiated with X-rays for analysis.

Aggregates in $\mathrm{H} 4$ cells have been proven to be positive for the Thioflavin-S staining (Lazaro et al. 2014). This characteristic points out the fact that these aggregates might display a fibrillar-like structure and maybe further STXM analysis, could be carried out on this model using longer distances between the sample and the detector to detect signal scattered at higher angles. In fact, the detector was placed at $5 \mathrm{~m}$ from the sample and in this setup only the SAXS signal could be recorded.

However these aggregates might not show a signal because they do not possess a structure that highly ordered enough to give a signal. The system is highly artificial as native aSyn without the EGFP fragment doesn't aggregate (McLean et al. 2001), thus indicating the need of the fragment that cannot be found in patients.

Moreover, Thioflavin-S can bind to different aSyn strains in an unspecific manner. Electron microscopy revealed that different aSyn strains have different structures (Bousset et al. 2013) and this leads also to different diffraction signal in Xrays while both the forms were able to bind Thioflavin S.

Taken together, SynT/ Synphilin-1 aggregates failed to show a signal in the darkfield of the STXM images. This is particularly important, because this model is frequently employed as in vitro aggregation model, also for the evaluation of "antiaggregative" substances. Given the fact, that the aggregates in this model are not visualized by STXM and thus may be less structured than aggregates in human tissue, the translation of data derived in this model to the human system must be seen with caution. 


\subsubsection{PD patients tissue shows characteristic peaks in the diffraction patterns at wide angles}

The lack of signal from the SynT/ Synphilin-1 aggregates in the H4 cell line could have been due to specifications of detection, but more likely they are due to an inherent feature of these structures. PD patients display as a hallmark of the disease the presence of LB that are mainly formed of aSyn. The LB are specific aggregations present only the diseased patients' brains and so far could not be recreated in laboratory conditions (Araki et al. 2015).

An analysis on the small-angle $x$-ray scattering (SAXS) and wide-angle $x$-ray scattering (WAXS) was performed on dried sections coming from snap frozen human SNpc, SNpr and cerebral peduncle zones of PD patients and CTR samples with a microbeam focus to evaluate electron density variations due to the aggregate presence, in order to characterize the LB structures.

X-ray diffraction on cells has been proven to be a powerful tool in understanding the orientation and the nature of subcellular structures like components of the cytoskeleton or microorganisms (Priebe et al. 2014; Bernhardt et al. 2016). Those studies were mostly looking at the anisotropy of molecules like actin and other filamentous proteins, which have an intrinsically defined structural orientation.

This should also be the case in LB, as they have been shown to be mostly made of fibrillar aSyn (Tofaris \& Spillantini 2007; Pieri et al. 2016). However, in the current analysis it was not possible to correlate the LB found in the adjacent sections stained with H\&E with the cells present in the native sections analyzed with $\mathrm{X}$-ray diffraction. The reason for this is that a single LB is about 3-5 $\mu \mathrm{m}$ in diameter (Dickson et al. 1989) and our sections had a thickness of $30 \mu \mathrm{m}$. Unfortunately, it was thus not possible finding a cell with a LB which than had to be precisely cut into half to be present in both adjacent sections.

However, even without imaging LBs, the analysis of X-ray diffraction patterns on the sections coming from PD patients showed interesting results with a clear signal in the WAXS range originating in specific areas of the scans. The angular average 
graph points out the presence of characteristic peaks at about $1.7 \mathrm{~nm}$ and $3.3 \mathrm{~nm}$. These distances are compatible with highly structured macromolecules such as protein aggregates. The same signal could be recorded by using two different kinds of support for the tissue: polypropylene and $\mathrm{Si}_{3} \mathrm{~N}_{4}$ membranes, which excludes unspecific signals originating from the support structure. Polypropylene can, to certain extent, give a signal in the SAXS and WAXS range (Nedkov \& Dobreva 2004), however the peaks given by this material are totally different than the ones given by the human tissue examined in this experiment.

The PD patient samples gave the highest signal intensity in the WAXS range. This signal cannot be due to an artifact of the polypropylene used as support, because the signal coming from the diffractions of tissue deposited on the $\mathrm{Si}_{3} \mathrm{~N}_{4}$ membranes is of the same intensity and nature.

Samples coming from Ctr were basically devoid of signals in the same diffraction range. Therefore it could be hypothesized that the highly ordered (crystallike) structure comes from aSyn aggregates. However, these highly ordered structures were mostly present in the extracellular space, while aSyn is mostly contained in the perykaryon (Spillantini et al. 1997; Braak et al. 2003). Recent studies on patients' tissue by the means of synchrotron FTIR micro-spectroscopy, show that LB are mostly made of $\beta$-structured fibrils that have a signal similar to the signal of fibrils made of recombinant aSyn (Araki et al. 2015). A fiber diffraction study carried out on fibrils of recombinant aSyn (Serpell et al. 2000), demonstrated that those fibrils have a periodicity of about $0.47 \mathrm{~nm}$ (for the monomer) and $1.1 \mathrm{~nm}$ (for the fibrils) and this is not comparable with the data that we obtained from our WAXS analysis.

The sections were negative for Amyloid-beta and phospho-Tau. The reason for that could have been due to the fact that the protocols for those staining are optimized for paraffin sections and not for cryo-sections, whose proteins are differently structured in absence of paraffin.

The signal arising from the highly ordered structures was coming from areas of the extracellular space, and it appears to be of similar dimensions as the areas of aggregated amyloid structures revealed by Bielschowski silver staining. The 
localization of the highly aggregated structures appear to be in the SNpc like the amyloid-like structure shown by the histological staining.

Despite the morphological similarity with Amyloid-beta aggregates, a comparison of the angular average peaks and the literature on the fiber diffraction of Amyloid-beta (Stroud et al. 2012) points out that it is unlikely for the highly ordered structures to arise from Amyloid-beta diffraction. The periodicity of Amyloid-beta fibers is reported with $0.47 \mathrm{~nm}$ for the interstrand distance and $1.0 \mathrm{~nm}$ for the intersheet distance.

It's important to keep in mind that Bielschowski silver staining isn't specific for a single kind of protein, but it reveals rather a protein conformation (Uchihara 2007). In fact, many aggregating proteins can be detected by this histological method; among those Amyloid-beta, phospho-Tau, aSyn and prion protein.

The distribution of another protein, super oxidase dismutase 1 (SOD1), could be similarly interesting to compare with X-ray diffraction data. This protein is crucial in the pathogenesis of familial amyotrophic lateral sclerosis (another neurodegenerative disease) and recently it has shown to be aggregating together with aSyn in a murine model of PD and also in the brains of PD patients (Helferich et al. 2015; Trist et al. 2016). Nonetheless also SOD1 diffraction pattern do not correlate with the signal recorded.

In summary, we detected an X-ray diffraction signal in the WAXS range in PD patients' brains tissue. It can be due to aggregating proteins in the brain as showed by Bielschowski stain. The comparison between the peaks of angular average and powder diffraction databases is still ongoing to identify the protein(s) involved. The origins of such a signal are challenging to define as in tissue there are several proteins interacting with each other and the powder diffraction databases contain mostly data for single proteins or well-defined supramolecular aggregates. Moreover the condition in the tissue might be different from the conditions in which the diffraction patterns of the proteins in the databases have been recorded. This leads to changes in protein folding making the research of the aggregating protein(s) even more challenging. 


\subsection{3. $\mathrm{Cu}$ and Fe quantification in PD patient's tissue}

PD patients' brains display a well-documented metal dys-homeostasis (Davies et al. 2014; Szczerbowska-Boruchowska et al. 2012). The first ever analyses executed on human samples, were extensively using histochemical staining methods to visualize the non-heme bound Fe in tissue by the so called "Perls staining". In Perls staining there is the reduction of $\mathrm{Fe}$ (III) to Fe(III) ferrocyanide that is a water-insoluble blue pigment (Perls 1867). Even if these methods are quite imprecise compared to today's knowledge (Meguro et al. 2007), they allowed to discover the Fe deposition PD patients' SN as early as 1924 by Lehermitte and colleagues (Lehermitte et al. 1924). Subsequently, this finding have been confirmed through other more sensitive techniques including mass spectrometry (Dexter et al. 1989) and MRI (Bartzokis et al. 1999).

In our experiment, we combined the study of the XRD together with XRF. XRF it's a technique that enables the quantification of several elements at once. Furthermore, the analysis was carried out doing a raster scan, thus enabling spatial localization of the elements. In comparison to histological stains, it is much more sensitive as it doesn't require any chemical reaction. In fact, it takes advantage of the physical-chemical properties of the elements themselves (Cesareo 1976). Moreover, due to high brilliancy of the synchrotron light source, the technique allows to have a single-cell resolution, which conventional mass-spectrometry systems could not achieve so far. It's remarkable to point out that the first approaches of XRF measures on PD tissues are about 50 years old and those results are still in line with more recent findings (Earle 1968; Dexter et al. 1989).

It had been previously published, that the neurons present in the human SN contain the highest amount of metals, while lower concentrations are reported to be in the extracellular space (Szczerbowska-Boruchowska et al. 2012; Surowka et al. 2016). The whole recorded elemental spectrum for all the scans was analyzed in order to find an element that enabled the discrimination between intracellular and extracellular space. The neurons analyzed in SN, are mostly NM positive cells, and previous reports, point out that the NM is rich in Fe, Cu and Zn (Zecca et al. 1996). Among the other elements, $\mathrm{Zn}$ was found to be the element that displayed the 
greatest concentration difference between intra and extracellular content. Zn was thus chosen as the element to discriminate between the two areas. It was therefore possible to set a threshold for Zn signal in order to extrapolate 4 different spectra: intracellular and extracellular content in the PD sample, and intracellular and extracellular content for the Ctr sample.

The elemental quantifications in the XRF-XRD experiment indicated that there was an accumulation of Fe in PD patients compared to the Ctr, especially in the extracellular compartment. The enrichment of Fe of the SN of PD patients was in line with what was previously published (Dexter et al. 1989; Bartzokis et al. 1999), However, the amount of Fe detected is too high compared to those previous studies. This might be due to a peculiarity of this particular PD patient examined. Unfortunately, in this analysis it was possible to compare only one PD patient and one Ctr due to the scarcity of time for performing the recording during beam-line.

Intriguingly, the Fe distribution in the extracellular space doesn't seem to follow a regular pattern. Indeed, there are in many scans some very bright spots of Fe. The reason of the presence of Fe deposits deserves a better characterization. The methods followed in sample preparation (like the special cryomatrix used, the temperature for cutting and the special Teflon-coated blades) should have prevented the displacement of metal between inside and outside the cell. The extracellular Fe depositions might thus indicate some still unexplored mechanism in the pathology.

The concentration of $\mathrm{Zn}$ results in a slight increase in the PD patients samples both in the intracellular and in the extracellular space. The value is nonetheless in line with previous reports (Szczerbowska-Boruchowska et al. 2012; Dexter et al. 1989)

On the other hand, $\mathrm{Cu}$ concentration in PD patients was shown to be reduced by about $50 \%$ in both the intracellular and intracellular space. This finding is similar to what has been recently shown (Davies et al. 2014). A higher Fe concentration while having a lower concentration of $\mathrm{Cu}$ is anyway in line with what has already been published regarding the counteracting effects of these two metals, and its substantially pointing out in the same direction of metal unbalance mechanisms in the etiopathology of PD. 
The analysis of metal content in the tissue samples not only is important to better understand the reasons of the dys-homeostasis of PD patients, but is also crucial because in in vitro experiments, aSyn interaction with several metals (like Fe and $\mathrm{Cu}$ ) results in a facilitation of its aggregation(Uversky et al. 2001; Paik et al. 1999; Binolfi et al. 2006). In this regard, the spatial distribution of the metals displayed by the XRF scans, could provide insights on aggregation processes in PD patients, by comparing the metals spatial distribution with the spatial distribution of the highly ordered structures.

The comparison of $\mathrm{Zn}$ distribution and total fluorescence with areas with highly ordered structures showed that the presence of the WAXS signal did not correlate with the cellular outline. This suggests that the highly ordered structures were mostly present in the extracellular space.

The comparison of Fe distribution and the distribution of highly ordered structures demonstrated that there was no correlation between the areas rich in Fe and the areas with WAXS signal. Fe was also distributed in extracellular deposits, and even if the highly ordered structures were also present in the outside the cells, again, there was no spatial correlation between Fe deposits and the areas with WAXS signal.

The same behavior could also be seen when the areas with highly ordered structured were compared with the $\mathrm{Cu}$ distribution. The concentration of $\mathrm{Cu}$ was very low in the areas with the signal as these areas belong to the PD sample, and there was not a clear correlation between the areas with the highest $\mathrm{Cu}$ signal and the areas with the WAXS signal.

The fact that the WAXS signal didn't correlate with the distributions of the transition metals in the scans, might mean that the formation of highly ordered structures seen here wasn't relying in the presence of Fe or Cu. Metals can foster aggregation but this is not the only possible mechanism. In fact, there are other mechanisms that can actively trigger the aggregation processes that could be responsible of the WAXS signals. For instance, the disrupted function of chaperone molecules (Doyle et al. 2013), as well as proteasome dysfunctions and aberrant transcriptional and post-transcriptional modifications (Tyedmers et al. 2010) can start the aggregation process in cells. Moreover, the most of NM neurons were dead 
and probably the aggregation processes started in the cells could have acted as "seeds" thus propagating the aggregation to other cells through endocytic vesicles trafficking pathways (Holmes \& Diamond 2012). Once the cells are dead they could release their content in the extracellular space thus leading to highly ordered structures seen in the WAXS range.

Moreover other proteins, other than the one (or the ones) that caused the WAXS signals might be responsible for the metal distribution in the brain. For example, transferrin is the main Fe carrier protein in the brain (Leitner \& Connor 2012) and coeruloplasmin has effect on Fe and Cu levels altogether (Patel et al. 2002). These proteins have a definite folding are not known to aggregate. Probably the metal distribution is affected more from this metal-trafficking proteins than for the ones that give the WAXS signal.

\subsubsection{Particle induced $\mathrm{X}$-ray emission on PD patient tissue reveals lower concentration of Cu compared to $\mathrm{Ctr}$}

To understand the role of LB in the metal homeostasis in the disease, we applied the PIXE on lyophilized sections from PD and Ctr. The aim was understanding whether metals are differentially distributed near LBs, e.g. close or not so close, clustered or homogeneous. To this, PIXE is a technique that allows quantitative measurements of different elements coupled with spatial information at the level of single cell resolution. Cells with and without LB in tissue coming from PD patients and Ctr subjects were comparing in this experiment. The lyophilization was chosen as the system that is capable to keep the metal distribution and concentration in the tissue at its best, i.e. as close as possible to the original conditions.

The metal content in the cells is not uniform in all the cells of the SN, but the NM positive cells display a higher metal content (Szczerbowska-Boruchowska et al. 2012) due to the presence of NM itself (Zecca et al. 1996). LBs are usually present in the NM positive cells, as these are the kind of cell that are most affected in the disease (Braak et al. 2003; Spillantini et al. 1997). For this reason, the compared cells were NM positive cells with LB and without LB from PD patients and Ctr. 
The absolute concentrations measured by PIXE were very similar for all the three kinds of cells for "house-keeping" elements like S, Ca, Zn and Fe. The fact that there were no differences in the Fe concentration between NM positive cells between Ctr and PD patients is in contrast with the findings of the previous experiment (see paragraph Errore. L'origine riferimento non è stata trovata.) and what has been previously reported in the literature (Dexter et al. 1989; Szczerbowska-Boruchowska et al. 2012; Hirsch et al. 1991). It's important to note that there was a large variation in the measures of $\mathrm{Fe}$ in NM positive cells of the PD patients' samples. Our results could thus simply reflect the limited number of patients measured: one had higher values of Fe concentrations while the others had lower values. Other samples coming from more patients are needed to obtain more robust results in Fe concentrations.

Conversely, NM positive cells from the PD patients analyzed displayed lower concentrations of $\mathrm{Cu}$ compared to $\mathrm{Ctr}$, which were similar to the previous experiment, and also similar to the values reported in literature (Davies et al. 2014). In fact, $\mathrm{Cu}$ amount in PD patients were about the half of the $\mathrm{Cu}$ amount in the Ctr samples.

Besides metal concentrations, also $\mathrm{P}$ and $\mathrm{K}$ concentrations appeared to be altered. P concentrations were lower in NM positive cells of PD patients compared to $\mathrm{Ctr}$, whereas $\mathrm{K}$ concentrations in the NM positive cells of the PD patients appeared higher compared to the Ctr, similarly to data previously reported (SzczerbowskaBoruchowska et al. 2012).

The values of the different analyzed elements $(\mathrm{P}, \mathrm{K}, \mathrm{Cl}$ and $\mathrm{Cu})$ were very similar for all kinds of NM positive cells coming from the PD patients' samples, regardless the presence of LB. One reason for that could be that the metal dys-homeostasis is independent of the LB presence. Moreover the data has to be seen with caution as it was possible to measure only four NM positive cells with LB and this number is likely too low, given also the difficulty in detecting LB. Technical difficulties to identify the same LB in both sections must be taken into account. In this case the sections were $40 \mu \mathrm{m}$ thick for both histology and for PIXE analysis compared to the thickness of a LB with $\sim 3$ - $5 \mu \mathrm{m}$ (Dickson et al. 1989). 
From this experiment, it was possible to confirm the metal dys-homeostasis in the SN of PD patients, with a special regard to the NM positive cells as they have a lower concentration of $\mathrm{Cu}$. The big variation in the concentrations of some elements (e.g. Fe, $\mathrm{P}$ and $\mathrm{Cl}$ ), pointed out that the experiment should be repeated with a higher number of patients in order to gain statistical robustness in special regard with LB measurement.

\subsection{Alpha-synuclein and metal handling}

The reason for the metal dys-homeostasis in the PD brains it's still unclear. Despite intense research efforts, it's still unknown whether aSyn can directly have an impact on this dys-homeostasis.

The analyzed post-mortem tissue comes directly from PD patients allowing for the closest-possible representation of the pathophysiological disease features. However, it usually shows tissue at the endpoint of the disease. Moreover, several genetic and environmental factors can alter disease progression in each patient. These external confounding factors may therefore hamper the identification of common patterns in the etiopathology of PD.

To study the effect of aSyn overexpression on metal amount and metal transport in a controlled setting, we used rat primary midbrain neurons (PNMs) transfected with adeno-associate virus vectors expressing human aSyn or Ctr.

\subsubsection{Alpha synuclein overexpression and Fe supply cause elemental dys-homeostasis in primary midbrain neurons}

In particular, we wanted to understand whether aSyn overexpression could cause a variation in the metal content in primary midbrain neurons (PMNs). Cultures transfected with aSyn in absence of Fe, displayed 50\% more Cu compared to cells expressing EGFP only. This fact is in line with a number of biophysical studies that report the direct binding of $\mathrm{Cu}$ to aSyn, and this finding suggests that the binding not only occurs in cell-free conditions but also in cells in vitro (Binolfi et al. 2012). There are two main high affinity sites for $\mathrm{Cu}(\mathrm{II})$ in aSyn in the $\mathrm{N}$-terminal part: one is in the 
first 5 amino-acids of the protein (Jackson \& Lee 2009) and the other is close to His50 (Binolfi et al. 2008). This binding could have a detrimental effect as it can increase the amount of aggregated aSyn (Paik et al. 1999; Uversky et al. 2001), but also could be a physiological feature of aSyn that could act as a ferrireductase in presence of $\mathrm{NADH}$ (Davies et al. 2011). This finding is in contrast with the values found for $\mathrm{Cu}$ in PD patients sections in both previous experiments, where there was a clear decrease in PD patients compared to Ctr (see Paragraph Errore. L'origine riferimento non è stata trovata. and Paragraph 4.1.4). This could be due to a regulatory mechanisms for Cu transport involving the blood-brain barrier that is not present when cells only are transfected. Also, only neuronal cells were studied in the in vitro experiments, while the tissue shows great heterogeneity in cell types. In addition, since patients with idiopathic PD were studied, an overexpression of aSyn does not occur.

When Fe(II) was added to the PMNs for 3 hours, the Cu concentration of the aSyn expressing cells still increased a bit, but the difference was not significant compared to the Ctr cells. Instead, the concentration of Fe was increased by $80 \%$ in cells expressing aSyn compared to Ctr transfected cells. This could be explained by a competition of $\mathrm{Cu}$ and Fe for the same importing protein: DMT1 (divalent metal transporter 1). As the name suggests, DMT1 is a protein that can import divalent metal cations inside the cell (Garrick et al. 2003). When the cells are without additional Fe supply, aSyn may sequester $\mathrm{Cu}$, since its affinity for this metal is very high. When Fe is administered in surplus to the medium, $\mathrm{Cu}$ and Fe compete for the same transporter and more Fe enters the cells due to a higher gradient.

Inside the cell, Fe(II) can bind aSyn. The affinity of aSyn for this metal is modest (Binolfi et al. 2006; Wang et al. 2011; Bharathi \& Rao 2007) but sufficient to justify the increased Fe content in aSyn transfected cells. Fe(II) can bind aSyn at the Cterminus in the region comprised between the amino acids 121 to 123 . This is not a specific binding region for Fe as other divalent metal can also bind in the same region. Among those, also $\mathrm{Mn}$ (II) can bind there and $\mathrm{Mn}$ can also be transported by DMT1 inside the cell. Intriguingly, in a similar study, when PMNs were supplemented with $\mathrm{Mn}$ in presence of aSyn overexpression (Dučić et al. 2015), the concentration of $\mathrm{Mn}$ was also increased in the cells overexpressing aSyn compared to Ctr-transfected cells. 
The fact that DMT1 and aSyn C-terminus binding site can play a role in the determination of the metal content in cells, is also supported by correlation analyses, which demonstrate a high correlation of intracellular concentrations of $\mathrm{Mn}$ and $\mathrm{Cu}$, and $\mathrm{Mn}$ and Fe (Dučić et al. 2015). These findings support the fact that aSyn might act as an intracellular metal storage protein by binding these metals.

\subsubsection{Alpha synuclein down-regulates CTR1 levels}

In addition to direct binding of metals, we investigated the possibility that aSyn overexpression could influence the levels of metal transporting proteins. To this, levels of mRNA and protein for several $\mathrm{Cu}$ and Fe transport proteins were analyzed. The high affinity copper uptake protein 1 (CTR1) enables $\mathrm{Cu}$ import inside the cell while ATP7a (responsible for Menke's disease) and APT7b (responsible for Wilson's disease) are two isoforms of the most important $\mathrm{Cu}$ exporting proteins. Divalent metal transporter 1 (DMT1) can bind all divalent metals and has an established role in the import of the divalent form of Fe, whereas Transferrin receptor (TfR) imports Fe that is bound to the plasmatic protein transferrin and Ferroportin 1 (Fpn1) is responsible for Fe export.

Upon aSyn overexpression, levels of mRNA were not significantly changed for any of these proteins. However, there was an inverse correlation between CTR1 expression and aSyn expression. This finding is particularly interesting as CTR1 was shown to be down-regulated also in PD patients tissue (Davies et al. 2014). One reason for this could be a feed-back loop triggered by intracellular $\mathrm{Cu}$ concentrations: since aSyn overexpression results in increased $\mathrm{Cu}$ concentrations CTR1 is downregulated in an attempt to compensate for increased Cu levels.

$\mathrm{Cu}$, as other metals, can exert a toxic effect through the Fenton reactions (see Chapter 1) and the feed-back regulation may attenuate oxidative stress due to excessive Cu presence. This idea is further supported by recent data, which show that in cells overexpressing CTR1 together with aSyn, $\mathrm{Cu}$ exposure was more toxic compared to cells transfected only with aSyn. Exogenous overexpression of CTR1 here was overruling the feed-back mechanism and the cells died because of oxidative stress (Anandhan et al. 2015). 


\subsection{In-vivo aggregation study: A53T mice and Fe supply}

In the final set of experiments, we simulated increased Fe exposition of the brain to understand the effects of Fe overload in an aSyn-based mouse model of PD, the aSyn.A53T mouse. In particular, we aimed to quantify the effects of Fe on aSyn aggregation and its behavioral consequences. To counteract the effects of Fe overload, we employed the clinically licensed chelator DFP.

\subsubsection{Behavioral analysis: rotarod examination}

Rotarod is a test for assessing motor learning abilities, coordination and balance of the animals. This test was performed with transgenic mice only as it was used for assessing the time of disease onset in those animals.

Similar to previous reports (Tatenhorst et al. 2016), the animals of the Ctr group as well as the DFPctr group (animals that received DFP only) learn within the first month how to perform the rotarod test implying that the DPF treatment is not detrimental for mice. In contrast, animals that have been treated with Fe but didn't receive the chelator treatment have difficulties in learning how to accomplish the test, suggesting that Fe overload attenuated their learning abilities. Previous reports have shown that the rotarod test has an impact on the learning abilities of the mice, in particularly in has to do with their synaptic plasticity (Peneder et al. 2011) that might be reduced after Fe administration. Moreover, wild-type mice treated with similar Fe overload paradigms, have difficulties in performing the radial arm maze at 3 months of age (Fredriksson et al. 1999; Fredriksson et al. 2000). The radial arm maze is a test that relies mostly on learning and spatial memory and mice use partially overlapping brain areas in both tasks.

The group of DPFFe (Fe-overloaded animals treated with DFP) performs the rotarod task as good as the Ctr animals, demonstrating that DFP is able to attenuate detrimental effects of $\mathrm{Fe}$ on behavior, as has already been shown in rats (Sripetchwandee et al. 2014). In this report, deficits in the Morris Water Maze Task of rats intoxicated with Fe could be partially rescued by DFP. Electrophysiological 
studies carried out on the hippocampi of these animals pointed out that long term potentiation was impaired in the Fe treated animals, which was partially rescued by DFP treatment (Sripetchwandee et al. 2014).

\subsubsection{Behavioral analysis: Novel object recognition}

The novel object recognition test (NOR) is a test for assessing several aspects of rodent cognition and relies upon the natural curiosity of the animals. When placed into the situation of exploring a familiar or a novel object, Ctr animals choose the latter and spend more time with that. The ability to choose between the new and the old object depends on the quality of the memory of the animal and on its physical conditions (Grayson et al. 2015).

The experiments on the A53T animals were performed at two time points: at post-natal day (P) 250 and disease onset. P 250 was chosen as the age at which the mice should start showing a diseased phenotype with the begin of accumulation of aggregates in the brain (Giasson et al. 2002). The time point of disease onset varies greatly among the animals, and is reached per definition when the mice display a weight loss $\geq 20 \%$ in a week or fail rotarod.

The results obtained at P 250 show a clear tendency for a decreased ability of the Fe-treated animals in preferring the new object over the old one, although statistical significance was not achieved due to limited animal numbers ( $p$-value $=$ $0.054)$.

At the disease onset time point, the differences leveled out, which most likely is due to the fact that the novelty seeking behavior in Ctr mice was not as pronounced anymore and therefore differences to other groups were attenuated. One could assume that progressive disease pathology at disease onset overrides the subtle behavioral alterations observed at the crosssectional P 250 time point.

It's noteworthy that at all time points, the distance run by the animals is the same in all groups. In a recent publication, A53T animals intoxicated with Fe, run shorter distances compared to the untreated A53T animals, which was attributed to hypo-activity induced by Fe (Billings et al. 2016). The intoxication model used in the 
aforementioned study is very similar to the one used in our case. In both experiments the animals were treated with Fe-carbonyl from p10 to p17, but the Fe concentration used by Billings and colleagues, was three times higher than the one used in our experiment: $120 \mathrm{mg} / \mathrm{kg}$ bw/day versus $40 \mathrm{mg} / \mathrm{kg}$ bw/day in our study. This higher concentration might have exacerbated the effects of Fe intoxication that we couldn't observe in our mice. The present concentration was chosen because we wanted to reproduce the Fe levels in brain present in the PD patients without overloading the liver (see (Dexter \& Jenner 2013)).

The NOR test performed here applied 10 minutes of memory latency (when the mouse was put back in the cage) between the time were it explored the familiar object and the time were the real test was recorded. Therefore the NOR test was indicative of short term memory. A study carried out in wild type Fe-intoxicated rats, however, reports significantly worse performances in the NOR test by Fe-treated rats, when it was carried in a long term memory regime ( $24 \mathrm{~h}$ memory latency) compared to a short term memory test where the differences were modest. In both the test, Fe intoxication was rescued by the administration of desferroxamine which is an Fe chelator, that chelates Fe(III) like DFP (de Lima et al. 2007; de Lima et al. 2005). The short term memory paradigm was applied in this study because it had previously shown to be able to allow the discernment of cognitive deficits in different murine models for PD. In fact, it was successfully used in MPTP mouse model (Sy et al. 2010), in the Thy-aSyn transgenic mouse model (Magen et al. 2012) and also in the A53T mouse model (Tatenhorst et al. 2016).

\subsubsection{Behavioral analysis: Catwalk analysis}

Catwalk is a very sensitive analysis system for assessing over 200 the gait parameters of a mouse that runs freely on a glass walkway. It has been extensively used in several rodent models of PD (Zhou et al. 2015; Tönges et al. 2012; Tatenhorst et al. 2014). Corresponding to the NOR test the animals were evaluated at P 250 and at disease onset.

At the time of disease onset, the results pointed out that the transgenic A53T animals are severely impaired in gait compared to age-matched WT-Ctr. This 
confirmed that the A53T system is reproducible and these results go in line with what was previously published in this model system (Tatenhorst et al. 2016).

The differences among the various groups of transgenic A53T animals are very mild: while there is a significant reduction of the parameter LF Max Intensity Mean in the Fe-treated group, several other parameters are only diminished by trend. Statistical significance was not reached due to limited animal number, which resulted from unexpected deaths in the different groups. Due to the long-term nature of the experimental setup, a replacement of these animals was not feasible. Nevertheless, the Catwalk analysis is in line with the other behavioral tests suggesting a deleterious effect of Fe intoxication on motor behavior, while the DFP and DFPFe groups tended to perform better.

\subsubsection{Analysis of survival}

Opposite to our initial assumptions, the Kaplan-Meier curve shows no differences in the survival between the groups and the average survival is similar to what has been already published in this model (Tatenhorst et al. 2016; Luk et al. 2012).

DFP, Fe administration, or their combination were not detrimental for the lifespan of the animals. The survival curve also suggests that overall the Fe intoxication paradigm used for this experiment was mild on general health conditions of the animals. Harsher paradigms for Fe intoxication and DFP administration have been previously used. For example, Kaur as well as Billings applied 120 mg/kg bw/day to mice from $P 10$ to $P 17$ in independent experiments, which is three times more than what was given in the present experiment (Kaur et al. 2003; Billings et al. 2016). As for DFP, our animals drank $10 \mathrm{mg} / \mathrm{kg}$ bw/day, and the concentration of the drug in the drinking water was $50 \mathrm{mg} / \mathrm{L}$, but it has been shown that mice tolerate well up to $1 \mathrm{~g} / \mathrm{L}$, which is 20 times as much (Song et al. 2014). However, the same amounts of DFP used in the present work have proven to be beneficial in a mouse model of PD treated of 6-OHDA (Workman et al. 2015) as well as in a Alzheimer model (Prasanthi et al. 2012) . 


\subsubsection{Aggregation analysis by dot blot}

The behavioral tests showed intriguing features regarding learning and motor coordination failure of the transgenic animals treated with Fe. Could this effect be due to a direct interaction of aSyn and Fe at the molecular level? Was this interaction having an effect of the aggregation state of aSyn in this model?

To answer these questions, we performed dot blot analyses on tissue lysates from the cortex that were digested with proteinase $\mathrm{K}(\mathrm{pK})$. Cortex was chosen from different brain areas as it showed the highest amount of aggregates in the original description of the model (Giasson et al. 2002). In order to select aggregated species, a digestion was carried out with pK, which digests the soluble forms of aSyn while it cannot cleave the aggregated forms. The dot-blot was chosen for visualization of aggregated species, because it permits to visualize protein aggregates independent of their size. In a regular western blot large-sized aggregates could escape analysis as they cannot enter the gel because of their dimensions. The detection antibody was directed against anti-human aSyn, because it has been already reported that there is no aggregation of murine aSyn in the model (Giasson et al. 2002; Tatenhorst et al. 2016)

The dot blot analysis shows a clear trend towards a reduction of aSyn aggregation for the animals that have been treated with DFP alone. This result goes in line with a previous publication, where a different chelator, Clioquinol, was used in the A53T mouse model (Finkelstein et al. 2016). In their experimental paradigm they proved that the amount of insoluble aSyn after Clioquinol treatment was significantly lower compared to controls. However, Clioquinol was administered at higher concentrations compared to DFP in our study: $37 \mathrm{mg} / \mathrm{kg}$ bw for Clioquinol against $10 \mathrm{mg} / \mathrm{kg}$ bw for DFP in our experiment. Moreover DFP has a very high affinity for Fe while being lower for other metals, while Clioquinol can chelate a lot of metals besides Fe, including $\mathrm{Cu}$ and $\mathrm{Zn}$ (Devos et al. 2014) and this may affect aSyn aggregation.

Also, our results show a trend for more aggregation in vivo in A53T animals supplemented with Fe and here the DFP treatment does not seem to have an effect 
on aggregation as the band intensities are comparable among the Fe and DFPFe groups. We can hypothesize that in contrast to the effects on intrinsic Fe concentrations (as observed in the DFP only group), even our mild Fe supplementation model (as in groups Fe and DFPFe) results in aggregation of aSyn, which cannot be counteracted by the given DFP concentrations. A preliminary doseresponse study for DFP was not performed here due to the long duration of the in vivo experiment, but might shed light on the effect of DFP dosage on aSyn aggregation.

In addition, it would be interesting to perform dot blot analyses of aggregated aSyn for the other brain areas, particularly considering the data obtained in the behavioral tests. The most interesting areas could be hippocampus, cerebellum and the basal ganglia as they are the areas demanded for motor coordination that are impaired in these animals.

\subsubsection{A53T mice and Fe intoxication in perspective}

The animals used for this experiment are syngenic and have been treated all in the same way within the same treatment group, and still the results display large variations. Nevertheless, there is a clear effect of Fe presence on learning abilities and motor coordination in mice. Since the coordination deficit it's rescued by the use of DFP, we assume that this effect is due to Fe intoxication, although non-chelating effects of DFP cannot be completely excluded.

Fe dys-homeostasis was previously studied in a MPTP model of PD in mice, where animals were treated with Clioquinol (Kaur et al. 2003). Here, the authors showed that Clioquinol administration increased the number of dopaminergic neurons and improved motor behavior. This is of particular interest since MPTP is essentially modelling oxidative stress and metabolic failure in PD. Chelator administration hinders oxidative stress formation due to metal presence. Clioquinol can chelate $\mathrm{Cu}, \mathrm{Zn}$ and Fe altogether thus exerting a protective action. Along the same research line is the study of Finkelstein and colleagues (Finkelstein et al. 2016). Here the same transgenic mouse model (aSyn.A53T) as in our study was used. Mice were treated with Clioquinol for a much shorter time compared to the present work, only 
for 8 months. The animals displayed a better performance in memory test like the Morris Water Maze and they had less aggregates in the brains. Since Clioquinol can chelate different metals, its action is much less specific compared to DFP. Moreover, they could show aggregates that were SDS-insoluble as early as after 8 months of age. In our setup at 8 months of age, animals had no detectable aggregate presence, as analyzed by $\mathrm{pK}$ resistance.

Another similar study to the one carried out in this work is the one performed by Billings and colleagues (Billings et al. 2016). Also in this case the aSyn.A53T animal model was used. Here, it is shown that Fe intoxication at high concentrations can reduce the motoric functions of the A53T animals that cannot be rescued by Clioquinol. The authors propose the use of chelators with higher Fe affinity (like DFP) for further studies. It has also to be taken into account that the studies carried out with Clioquinol, despite showing promising results, would never be translated into clinics. The compound has proven to be highly neurotoxic in humans and at the moment it is employed only as a topical remedy for fungal infections (Mao \& Schimmer 2008). Moreover we wanted to have a paradigm similar to the $\mathrm{Fe}$ intoxication in the patients the difference in the amount of Fe in the PD patients' brains compared to is relatively low (see (Dexter \& Jenner 2013)).

The Fe intoxication paradigm used in this study aimed to be as close as possible to the real condition in patients, avoiding extreme toxic effect of an excessive Fe overload and using a drug that can be used also in the clinics. Therefore the information given by this model is very valuable for the future use in therapy. 


\section{Conclusions}

The three experiments carried out in the present work shed a light on the role of Fe in PD, particularly its role in aSyn pathology. They also showed that X-ray imaging techniques and conventional biological paradigms can be used synergistically in providing insights in this interaction.

In particular, it was possible to analyze for the first time the signal of highly ordered structures directly in the tissue coming from PD patients and to correlate this signal with histological stains and metal distribution. The quantification of the trace metal content in the patients' tissue was in line with other reports and it reflected the metal dys-homeostasis in PD.

The data displayed further that aSyn was able to modify the metal content in the cells and it had an effect on the content either in presence or in absence of excessive Fe supply, thus confirming the tight connection between this protein and metals.

In mice, Fe intoxication in combination with aSyn aggregation has shown to be causative of motor learning deficits that could be rescued by a chelator able to cross the blood brain barrier: DFP. This implies that iron chelators could be used as an additional therapy for future treatment of the disease. DFP is currently being tested in clinical trials on PD patients in combination with conventional therapies. Early results are promising especially when the drug is administered at the first stage of the disease.

More research is needed to fully understand the interaction that occurs between aSyn and metals, but it's definitely a promising field to gather insights on the pathophysiology of the disease for possible translational perspectives in the search for new therapeutic opportunities. 


\section{Summary}

Parkinson's disease (PD) is the second most common neurodegenerative disease worldwide. It's a progressive disease that can be treated only symptomatically and its causes are still not fully understood. Two main factors have shown to play a pivotal role in the etiopathology of PD: the metal dys-homeostasis and the presence of aggregated protein structures (Lewy Bodies) that are mainly composed of the protein alpha-synuclein (aSyn).

The present work had the aim of better characterizing the role of the interaction between aSyn and iron (Fe) in PD. A combination of X-ray-based imaging techniques together with cell-biology methods and animal models was applied for studying this interactions.

The analysis showed for the first time the presence of mostly extracellularly located highly ordered structures in brain tissue of PD patients using $x$-ray diffraction techniques. The distribution and quantification of the trace metals in the samples exhibited an increased Fe content in line with previous data on metal dyshomeostasis in PD.

Particle-induced X-ray emission was able to prove that aSyn overexpression affects the metal content in primary midbrain neurons in presence and in absence of excessive Fe supply. Furthermore, overexpression of aSyn changed the expression of metal transport proteins.

A murine model of aSyn aggregation (prnp.aSyn.A53T) in combination with mild Fe intoxication showed that this interaction leaded to the appearance of motor learning deficits. In this murine paradigm DFP, a Fe chelator that is able to cross the blood brain barrier, could rescue the deleterious effects of Fe intoxication. This suggested that Fe chelators could be combined with the symptomatic therapies for PD for future treatments as first clinical trials with this drug also indicate.

This work used a combination of biological and X-ray imaging techniques to shed a light on the interaction of aSyn and Fe and thus contributed to a deeper understanding of PD pathomechanisms and future disease-modifying translational therapies. 


\section{References}

Alpen, E.L., 1998. Radiation biophysics, second edition, Academic Press.

Anandhan, A. et al., 2015. Overexpression of alpha-synuclein at non-toxic levels increases dopaminergic cell death induced by copper exposure via modulation of protein degradation pathways. Neurobiology of Disease, 81, pp.76-92. Available at: http://dx.doi.org/10.1016/j.nbd.2014.11.018.

Apetri, M.M. et al., 2006. Secondary structure of alpha-synuclein oligomers: Characterization by Raman and atomic force microscopy. Journal of Molecular Biology, 355(1), pp.63-71.

Araki, K. et al., 2015. Synchrotron FTIR micro-spectroscopy for structural analysis of Lewy bodies in the brain of Parkinson's disease patients. Scientific reports, 5(January), p.17625.

Available

at: http://www.nature.com/srep/2015/151201/srep17625/full/srep17625.html.

Avwioro, G., 2011. Histochemical uses of Haematoxylin - A review. Jpcs, 1(April-June), pp.24-34. Available

at: http://www.arpapress.com/Volumes/JPCS/Vol1/JPCS_1_05.pdf.

Barbour, R. et al., 2008. Red blood cells are the major source of alpha-synuclein in blood. Neuro-degenerative diseases, 5(2), pp.55-9. Available at: http://www.ncbi.nlm.nih.gov/pubmed/18182779.

Bartzokis, G. et al., 1999. MRI evaluation of brain iron in earlier- and later-onset Parkinson's disease and normal subjects. Magnetic Resonance Imaging, 17(2), pp.213-222.

Bernhardt, M. et al., 2016. X-Ray Micro- and Nanodiffraction Imaging on Human Mesenchymal Stem Cells and Differentiated Cells. Biophysical Journal, 110(3), pp.680-690. Available at: http://dx.doi.org/10.1016/j.bpj.2015.12.017.

Berrocal, R. et al., 2014. $\alpha$-Synuclein Misfolding Versus Aggregation Relevance to Parkinson's Disease: Critical Assessment and Modeling. Molecular neurobiology, pp.1417-1431. Available at: http://www.ncbi.nlm.nih.gov/pubmed/25139280. 
Bevins, R. a \& Besheer, J., 2006. Object recognition in rats and mice: a one-trial nonmatching-to-sample learning task to study "recognition memory". Nature protocols, 1(3), pp.1306-1311. Available at: http://dx.doi.org/10.1038/nprot.2006.205.

Bharathi \& Rao, K.S.J., 2007. Thermodynamics imprinting reveals differential binding of metals to $\alpha$-synuclein: Relevance to parkinson's disease. Biochemical and Biophysical Research Communications, 359, pp.115-120.

Billings, J.L. et al., 2016. Effects of Neonatal Iron Feeding and Chronic Clioquinol Administration on the Parkinsonian Human A53T Transgenic Mouse. ACS Chemical Neuroscience, 7(3), pp.360-366.

Binolfi, A. et al., 2012. Bioinorganic chemistry of copper coordination to alpha-synuclein: Relevance to Parkinson's disease. Coordination Chemistry Reviews, 256(19-20), pp.2188-2201. Available at: http://dx.doi.org/10.1016/j.ccr.2012.05.004.

Binolfi, A. et al., 2008. Site-specific interactions of $\mathrm{Cu}(\mathrm{II})$ with alpha and beta-synuclein: bridging the molecular gap between metal binding and aggregation. J Am Chem Soc, 130(35), pp.11801-11812. Available at: http://eutils.ncbi.nlm.nih.gov/entrez/eutils/elink.fcgi?dbfrom=pubmed\&id=18693 689\&retmode=ref\&cmd=prlinks\%5Cnpapers3://publication/doi/10.1021/ja80349 $4 v$.

Binolfi, a et al., 2006. Interaction of alpha-synuclein with divalent metal ions reveals key differences: a link between structure, binding specificity and fibrillation enhancement 21. J.Am.Chem.Soc., 128(30), pp.9893-9901.

Bousset, L. et al., 2013. Structural and functional characterization of two alpha-synuclein strains. Nature communications, 4, p.2575. Available at: http://www.pubmedcentral.nih.gov/articlerender.fcgi?artid=3826637\&tool=pmc entrez\&rendertype=abstract.

Braak, H. et al., 2003. Staging of brain pathology related to sporadic Parkinson's disease. Neurobiology of Aging, 24(2), pp.197-211.

Breydo, L. \& Uversky, V., 2011. Role of metal ions in aggregation of intrinsically disordered proteins in neurodegenerative diseases. Metallomics, 3(11), pp.1163- 
80.

Available

at:

http://www.ncbi.nlm.nih.gov/pubmed/21869995\%5Cnhttp://pubs.rsc.org/en/con tent/articlehtml/2011/mt/c1mt00106j.

Burke, R.E. \& O'Malley, K., 2013. Axon Degeneration in Parkinson â€ $€^{\mathrm{TM}}$ s Disease. Exp Neurol., pp.72-83.

Burré, J. et al., 2010. Alpha-synuclein promotes SNARE-complex assembly in vivo and in vitro. Science (New York, N.Y.), 329(5999), pp.1663-7. Available at: http://www.pubmedcentral.nih.gov/articlerender.fcgi?artid=3235365\&tool=pmc entrez\&rendertype=abstract.

Butler, D. a et al., 1988. Scrapie-infected murine neuroblastoma cells produce proteaseresistant prion proteins. Journal of virology, 62(5), pp.1558-1564.

Campbell, R.C., 1989. Statistics for Biologists. 3rd ed. C. U. Press, ed., Cambridge: Cambridge Books Online.

Carboni, E. \& Lingor, P., 2015. Insights on the interaction of alpha-synuclein and metals in the pathophysiology of \{Parkinson\}'s disease. Metallomics, 7(3), pp.395-404. Available at: http://dx.doi.org/10.1039/C4MT00339J.

Castellani, R.J. et al., 2000. Sequestration of iron by Lewy bodies in Parkinson's disease. Acta neuropathologica, 100(2), pp.111-114.

Cesareo, R., 1976. Trace elements analysis in biological samples by radioisotopic X-ray fluorescence. European Journal of Nuclear Medicine, 1(1), pp.49-56.

Chen-Plotkin, A.S., 2014. Unbiased approaches to biomarker discovery in neurodegenerative diseases. Neuron, 84(3), pp.594-607.

Chen, S.W. et al., 2015. Structural characterization of toxic oligomers that are kinetically trapped during $\alpha$-synuclein fibril formation. Proceedings of the National Academy of Sciences of the United States of America, 112(16), pp.E1994-2003. Available at: http://www.pubmedcentral.nih.gov/articlerender.fcgi?artid=4413268\&tool=pmc entrez\&rendertype=abstract.

Chomczynski, P., 1993. Dataset · April 2016. Biotechniques., 15(3), pp.532-4, 536-7.

Chomczynski, P. \& Sacchi, N., 1987. Single-step method of RNA isolation by acid 
guanidinium thiocyanate-phenol-chloroform extraction. Analytical Biochemistry, 162(1), pp.156-159.

Connolly, B.S. \& Lang, A.E., 2014. Pharmacological Treatment of Parkinson Disease. Jama, 311(16), p.1670.

Davies, K.M. et al., 2014. Copper pathology in vulnerable brain regions in Parkinson's disease. Neurobiology of Aging, 35(4), pp.858-866. Available at: http://linkinghub.elsevier.com/retrieve/pii/S0197458013004363.

Davies, P., Moualla, D. \& Brown, D.R., 2011. Alpha-synuclein is a cellular ferrireductase. PloS one, 6(1), p.e15814. Available at: http://www.pubmedcentral.nih.gov/articlerender.fcgi?artid=3018422\&tool=pmc entrez\&rendertype=abstract.

Deng, H. \& Yuan, L., 2014. Genetic variants and animal models in SNCA and Parkinson disease. Ageing Research Reviews, 15(1), pp.161-176. Available at: http://dx.doi.org/10.1016/j.arr.2014.04.002.

Devos, D. et al., 2014. Targeting chelatable iron as a therapeutic modality in Parkinson's disease. Antioxidants \& redox signaling, 21(2), pp.195-210. Available at: http://www.pubmedcentral.nih.gov/articlerender.fcgi?artid=4060813\&tool=pmc entrez\&rendertype=abstract.

Dexter, D.T. et al., 1989. Increased nigral iron content and alterations in other metal ions occurring in brain in Parkinson's disease. Journal of neurochemistry, 52(6), pp.1830-1836.

Dexter, D.T. \& Jenner, P., 2013. Parkinson disease: from pathology to molecular disease mechanisms. Free Radic Biol Med, 62, pp.132-144. Available at: http://www.ncbi.nlm.nih.gov/pubmed/23380027\%5Cnhttp://ac.elscdn.com/S0891584913000282/1-s2.0-S0891584913000282main.pdf?_tid=1d968706-6389-11e4-907a00000aab0f6b\&acdnat=1415040308_2b2424a02dea1981c72c853d7a02399d.

Dias, V., Junn, E. \& Mouradian, M.M., 2014. The Role of Oxidative Stress in Parkinson's Disease. J Parkinsons Dis, 3(4), pp.461-491. 
Dickson, D.W. et al., 1989. Diffuse Lewy body disease: light and electron microscopic immunocytochemistry of senile plaques. Acta Neuropathologica, 78(6), pp.572584.

Doyle, S.M., Genest, O. \& Wickner, S., 2013. Protein rescue from aggregates by powerful molecular chaperone machines. Nature reviews. Molecular cell biology, 14(10), pp.617-29. Available at: http://www.ncbi.nlm.nih.gov/pubmed/24061228.

Dučić, T. et al., 2015. Alpha-Synuclein Regulates Neuronal Levels of Manganese and Calcium. ACS Chemical Neuroscience, 6(10), pp.1769-1779.

Dunnett, S.B. \& Björklund, A., 1999. Prospects for new restorative and neuroprotective treatments in Parkinson's disease. Nature, 399(6738 Suppl), pp.A32-A39.

Earle, K.M., 1968. Studies on Parkinson's disease including x-ray fluorescent spectroscopy of formalin fixed brain tissue. J Neuropathol Exp Neurol., 27(1), pp.114.

Emden, V., Helmut, F. \& Emden, H. van, 2008. Statistics for terrified biologists, Available at: http://centaur.reading.ac.uk/9910/.

Finkelstein, D.I. et al., 2016. Clioquinol Improves Cognitive, Motor Function, and Microanatomy of the Alpha-Synuclein hA53T Transgenic Mice. ACS Chemical Neuroscience, 7(1), p.acschemneuro.5b00253. Available at: http://pubs.acs.org/doi/10.1021/acschemneuro.5b00253.

Fleming, S.M., Fernagut, P.-O. \& Chesselet, M.-F., 2005. Genetic mouse models of parkinsonism: strengths and limitations. NeuroRx: the journal of the American Society for Experimental NeuroTherapeutics, 2(3), pp.495-503. Available at: http://www.pubmedcentral.nih.gov/articlerender.fcgi?artid=1144493\&tool=pmc entrez\&rendertype=abstract.

Fornai, F. et al., 2005. Parkinson-like syndrome induced by continuous MPTP infusion: convergent roles of the ubiquitin-proteasome system and alpha-synuclein. Proceedings of the National Academy of Sciences of the United States of America, 102(9), pp.3413-3418. Available at: http://www.pubmedcentral.nih.gov/articlerender.fcgi?artid=552938\&tool=pmce ntrez\&rendertype=abstract. 
Fredriksson, A. et al., 2000. Maze learning and motor activity deficits in adult mice induced by iron exposure during a critical postnatal period. Brain research. Developmental brain research, 119(1), pp.65-74. Available at: http://www.ncbi.nlm.nih.gov/pubmed/10648873.

Fredriksson, A. et al., 1999. Neonatal iron exposure induces neurobehavioural dysfunctions in adult mice. Toxicology and applied pharmacology, 159(1), pp.2530.

Galvan, A. \& Wichmann, T., 2008. Pathophysiology of parkinsonism. Clin Neurophysiol., 119(7), pp.1459-1474.

Garrick, M.D. et al., 2003. DMT1: A mammalian transporter for multiple metals. BioMetals, 16(1), pp.41-54.

Gianoncelli, a et al., 2015. Soft X-Ray Microscopy Radiation Damage On Fixed Cells Investigated With Synchrotron Radiation FTIR Microscopy. Scientific reports, 5(April), p.10250. Available at: http://www.pubmedcentral.nih.gov/articlerender.fcgi?artid=4431353\&tool=pmc entrez\&rendertype=abstract.

Giasson, B.I. et al., 2002. Neuronal $\alpha$-Synucleinopathy with Severe Movement Disorder in Mice Expressing A53T Human $\alpha$-Synuclein. Neuron, 34(4), pp.521-533. Available at: http://www.sciencedirect.com/science/article/pii/S0896627302006827.

Graewert, M.A. \& Svergun, D.I., 2013. Impact and progress in small and wide angle X-ray scattering (SAXS and WAXS). Current Opinion in Structural Biology, 23(5), pp.748754. Available at: http://dx.doi.org/10.1016/j.sbi.2013.06.007.

Graham, D.R. \& Sidhu, A., 2010. Mice expressing the A53T mutant form of human alphasynuclein exhibit hyperactivity and reduced anxiety-like behavior. Journal of Neuroscience Research, 1783, p.NA-NA. Available at: http://doi.wiley.com/10.1002/jnr.22331.

Grayson, B. et al., 2015. Assessment of disease-related cognitive impairments using the novel object recognition (NOR) task in rodents. Behavioural Brain Research, 285, pp.176-193. Available at: http://dx.doi.org/10.1016/j.bbr.2014.10.025. 
Grolez, G. et al., 2015. Ceruloplasmin activity and iron chelation treatment of patients with Parkinson's disease. BMC neurology, 15, p.74. Available at: http://www.pubmedcentral.nih.gov/articlerender.fcgi?artid=4429376\&tool=pmc entrez\&rendertype=abstract.

Helferich, A.M. et al., 2015. $\alpha$-synuclein interacts with SOD1 and promotes its oligomerization. Molecular neurodegeneration, 10(1), p.66. Available at: http://www.ncbi.nlm.nih.gov/pubmed/26643113.

Hirsch, E.C. et al., 1991. Iron and Aluminum Increase in the Substantia Nigra of Patients with Parkinson's Disease: An X-Ray Microanalysis. Journal of Neurochemistry, 56(2), pp.446-451. Available at: http://www.ncbi.nlm.nih.gov/pubmed/1988548\%5Cnhttp://doi.wiley.com/10.11 11/j.1471-4159.1991.tb08170.x.

Holmes, B.B. \& Diamond, M.I., 2012. Cellular mechanisms of protein aggregate propagation. Current Opinion in Neurology, 25(6), p.1.

Iranzo, A., 2013. Parkinson Disease and Sleep: Sleep-Wake Changes in the Premotor Stage of Parkinson Disease; Impaired Olfaction and Other Prodromal Features. Current Neurology and Neuroscience Reports, 13(9), p.373. Available at: http://link.springer.com/10.1007/s11910-013-0373-0.

Irvine, G. \& El-Agnaf, O., 2008. <Title/>. Molecular Medicine, 14(7-8), p.1. Available at: http://www.molmed.org/content/pdfstore/451_464.Irvine.00100.PDF.

Jackson-Lewis, V. \& Przedborski, S., 2007. Protocol for the MPTP mouse model of Parkinson's disease. Nature Protocols, 2(1), pp.141-151. Available at: http://www.nature.com/doifinder/10.1038/nprot.2006.342.

Jackson, M.S. \& Lee, J.C., 2009. Identification of the minimal copper(II)-binding alphasynuclein sequence. Inorg Chem., 48(19), pp.22-9303-7.

Jagmag, S.A. et al., 2016. Evaluation of models of Parkinson's disease. Frontiers in Neuroscience, $9(\mathrm{JAN})$.

Johansson, S.A.E., Campbell, J.L. \& Malmqvist, K.G., 1995. Particle-Induced X-Ray Emission Spectrometry (Pixe), John Wiley \& Sons Inc. 
Jones, B.J. \& Roberts, D.J., 1968. The quantiative measurement of motor inco-ordination in naive mice using an acelerating rotarod. The Journal of pharmacy and pharmacology, 20(4), pp.302-304.

Kandel, E.R., Schwartz, J.H. \& Jessell, T.M., 2012. Principles of Neural Science,

Kasten, M. \& Klein, C., 2013. The many faces of alpha-synuclein mutations. Movement Disorders, 28(6), pp.697-701. Available at: http://doi.wiley.com/10.1002/mds.25499.

Kaur, D. et al., 2003. Genetic or Pharmacological Iron Chelation Prevents MPTP-Induced Neurotoxicity In Vivo. Neuron, 37(6), pp.899-909. Available at: http://www.sciencedirect.com/science/article/pii/S0896627303001260.

Klein, C. \& Westenberger, A., 2012. Genetics of Parkinson's disease. Cold Spring Harb Perspect Med., 2.

Klucken, J. et al., 2004. Hsp70 reduces ??-synuclein aggregation and toxicity. Journal of Biological Chemistry, 279(24), pp.25497-25502.

Knöferle, J. et al., 2010. TGF- $\beta 1$ enhances neurite outgrowth via regulation of proteasome function and EFABP. Neurobiology of Disease, 38(3), pp.395-404. Available at: http://dx.doi.org/10.1016/j.nbd.2010.02.011.

Laemmli, U.K., 1970. Cleavage of structural proteins during the assembly of the head of bacteriophage T4. Nature, 227(5259), p.680-5.

Langston, J.W., Langston, E.B. \& Irwin, I.I., 1984. MPTP-induced parkinsonism in human and non-human primates--clinical and experimental aspects. Acta Neurol Scand Suppl., 100, pp.49-54.

Lazaro, D.F. et al., 2014. Systematic Comparison of the Effects of Alpha-synuclein Mutations on Its Oligomerization and Aggregation. PLoS Genetics, 10(11).

Leitner, D.F. \& Connor, J.R., 2012. Functional roles of transferrin in the brain. Biochimica et Biophysica Acta - General Subjects, 1820(3), pp.393-402. Available at: http://dx.doi.org/10.1016/j.bbagen.2011.10.016.

Lhermitte, J., Kraus, W.M. \& McAlpine, D., 1924. The Occurence of abnormal deposits of iron in the brain in parkinsonism with special reference to its localisation. The 
Journal of Neurology and Psychopathology, V(19), pp.195-208.

Likhite, N. \& Warawdekar, U.M., 2011. A unique method for isolation and solubilization of proteins after extraction of RNA from tumor tissue using Trizol. Journal of Biomolecular Techniques, 22(1), pp.37-44.

de Lima, M.N.M. et al., 2007. Desferoxamine reverses neonatal iron-induced recognition memory impairment in rats. European Journal of Pharmacology, 570(1-3), pp.111114.

de Lima, M.N.M. et al., 2005. Recognition memory impairment and brain oxidative stress induced by postnatal iron administration. European Journal of Neuroscience, 21(9), pp.2521-2528. Available at: http://doi.wiley.com/10.1111/j.14609568.2005.04083.x.

Lingor, P., Unsicker, K. \& Krieglstein, K., 2000. GDNF and NT-4 protect midbrain dopaminergic neurons from toxic damage by iron and nitric oxide. Experimental neurology, 163(1), pp.55-62. Available at: http://www.ncbi.nlm.nih.gov/pubmed/10785444.

Luk, K.C. et al., 2012. Intracerebral inoculation of pathological $\alpha$-synuclein initiates a rapidly progressive neurodegenerative $\alpha$-synucleinopathy in mice. The Journal of experimental medicine, 209(5), pp.975-86. Available at: http://jem.rupress.org/content/209/5/975.long.

Magen, l. et al., 2012. Cognitive deficits in a mouse model of pre-manifest Parkinson's disease. Eur J Neurosci., 35(6), pp.870-882.

Makrigiannakis, A. et al., 1999. N-cadherin-mediated human granulosa cell adhesion prevents apoptosis: a role in follicular atresia and luteolysis? The American journal of pathology, 154(5), pp.1391-406. Available at: http://www.pubmedcentral.nih.gov/articlerender.fcgi?artid=1866595\&tool=pmc entrez\&rendertype=abstract.

Mao, X. \& Schimmer, A.D., 2008. The toxicology of Clioquinol. Toxicology Letters, 182(13), pp.1-6.

Mayeux, R., 2006. Genetic epidemiology of Alzheimer disease. Alzheimer 
Dis.Assoc.Disord., 20(0893-0341 (Print)), pp.S58-S62.

McLean, P.J., Kawamata, H. \& Hyman, B.T., 2001. ??-Synuclein-enhanced green fluorescent protein fusion proteins form proteasome sensitive inclusions in primary neurons. Neuroscience, 104(3), pp.901-912.

Meguro, R. et al., 2007. Nonheme-iron histochemistry for light and electron microscopy: a historical, theoretical and technical review. Arch Histol Cytol., 70(1), pp.1-19.

Metz, G.A. et al., 2005. The unilateral 6-OHDA rat model of Parkinson's disease revisited: An electromyographic and behavioural analysis. European Journal of Neuroscience, 22(3), pp.735-744.

Moretto, A. \& Colosio, C., 2011. Biochemical and toxicological evidence of neurological effects of pesticides: The example of Parkinson's disease. NeuroToxicology, 32(4), pp.383-391. Available at: http://dx.doi.org/10.1016/j.neuro.2011.03.004.

Morris, A.M. \& Finke, R.G., 2009. alpha-Synuclein aggregation variable temperature and variable $\mathrm{pH}$ kinetic data: A re-analysis using the Finke-Watzky 2-step model of nucleation and autocatalytic growth. Biophysical Chemistry, 140(1-3), pp.9-15. Available at: http://dx.doi.org/10.1016/j.bpc.2008.11.003.

Morrison, G.R. \& Browne, M.T., 1992. Dark-field imaging with the scanning transmission x-ray microscope. Review of Scientific Instruments, 63(1), pp.611-614.

Nedkov, E. \& Dobreva, T., 2004. Wide and small-angle X-ray scattering study of isotactic polypropylene gamma irradiated in bulk. European Polymer Journal, 40(11), pp.2573-2582.

ONU, 2015. World population, ageing. , p.164. Available at: www.un.org/.../population/.../WPA2009/WPA2009.

Oprandy, J.J., Olson, J.G. \& Scott, T.W., 1988. A rapid dot immunoassay for the detection of serum antibodies to eastern equine encephalomyelitis and St. Louis encephalitis viruses in sentinel chickens. Am J Trop Med Hyg., 38(1), pp.181-6.

Ortega, R. et al., 2015. $\alpha$-Synuclein Over-Expression Induces Increased Iron Accumulation and Redistribution in Iron-Exposed Neurons. Molecular neurobiology, p.[Epub ahead of print]. Available at: 
http://www.ncbi.nlm.nih.gov/pubmed/25833099.

Oung, Q.W. et al., 2015. Technologies for Assessment of Motor Disorders in Parkinson's Disease: A Review,

Paik, S.R. et al., 1999. Copper(II)-induced self-oligomerization of alpha-synuclein. The Biochemical journal, 340 ( Pt 3, pp.821-828.

Parkinson, J., 1817. An Essay on the Shaking Palsy.

Patel, B.N. et al., 2002. Ceruloplasmin regulates iron levels in the CNS and prevents free radical injury. The Journal of neuroscience, 22(15), pp.6578-6586.

Peelaerts, W. et al., 2015. $\alpha$-Synuclein strains cause distinct synucleinopathies after local and systemic administration. Nature, 522, pp.340-344. Available at: http://www.ncbi.nIm.nih.gov/pubmed/26061766.

Peneder, T.M. et al., 2011. Chronic exposure to manganese decreases striatal dopamine turnover in human alpha-synuclein transgenic mice. Neuroscience, 180, pp.280-92. Available at: http://www.ncbi.nlm.nih.gov/pubmed/21333719.

Perez, C., Tong, Y. \& Guo, M., 2008. Iron Chelators as Potential Therapeutic Agents for Parkinson's Disease. Current bioactive compounds, 4(3), pp.150-158.

Perls, M., 1867. Nachweis von Eisenoxyd in gewissen Pigmenten. Virchows. Arch. Pathol. Anat., 39, pp.42-8.

Pieri, L. et al., 2016. Cellular response of human neuroblastoma cells to $\alpha$-synuclein fibrils, the main constituent of Lewy bodies. Biochimica et Biophysica Acta-General Subjects, 1860(1), pp.8-19. Available at: http://dx.doi.org/10.1016/j.bbagen.2015.10.007.

Polymeropoulos, M.H., 1997. Mutation in the -Synuclein Gene Identified in Families with Parkinson's Disease. Science, 276(5321), pp.2045-2047.

Prasanthi, J. et al., 2012. Deferiprone reduces amyloid- $\beta$ and tau phosphorylation levels but not reactive oxygen species generation in hippocampus of rabbits fed a cholesterol-enriched diet. J Alzheimers Dis., 30(1), pp.167-182.

Priebe, M. et al., 2014. Scanning X-Ray Nanodiffraction on Dictyostelium discoideum. 
Biophysj, 107(11), pp.2662-2673. Available at: http://dx.doi.org/10.1016/j.bpj.2014.10.027.

Pringsheim, T. et al., 2014. The prevalence of Parkinson's disease: A systematic review and meta-analysis. Movement Disorders, 29(13), pp.1583-1590.

Recasens, A. \& Dehay, B., 2014. Alpha-synuclein spreading in Parkinsonâ€ $€^{\mathrm{TM}_{S}}$ disease. Frontiers in Neuroanatomy, 8(December), pp.1-9. Available at: http://journal.frontiersin.org/article/10.3389/fnana.2014.00159/abstract.

Rodriguez-Fernandez, L., Miranda, J. \& Oliver, A., 1994. Characterization of a Si(Li) detector for PIXE analysis. Journal of X-Ray Science and Technology, 4(3), pp.221246.

Saal, K.-A. et al., 2015. AAV.shRNA-mediated downregulation of ROCK2 attenuates degeneration of dopaminergic neurons in toxin-induced models of Parkinson's disease in vitro and in vivo. Neurobiology of disease, 73, pp.150-62. Available at: http://www.ncbi.nlm.nih.gov/pubmed/25283984.

Seeck, O.H. \& Murphy, B., 2015. X-Ray Diffraction: Modern Experimental Techniques Pan Stanfo.,

Seibert, J. a. \& Boone, J.M., 2005. X-ray imaging physics for nuclear medicine technologists. Part 2: X-ray interactions and image formation. Journal of nuclear medicine technology, 33(1), pp.3-18.

Serpell, L.C. et al., 2000. Fiber diffraction of synthetic alpha-synuclein filaments shows amyloid-like cross-beta conformation. Proceedings of the National Academy of Sciences of the United States of America, 97(9), pp.4897-4902.

Song, D. et al., 2014. The oral iron chelator deferiprone protects against systemic iron overload-induced retinal degeneration in hepcidin knockout mice. Investigative Ophthalmology and Visual Science, 55(7), pp.4525-4532.

Spillantini, M.G. et al., 1997. Alpha-synuclein in Lewy bodies. Nature, 388(6645), pp.839-840.

Sripetchwandee, J. et al., 2014. Combined therapy of iron chelator and antioxidant completely restores brain dysfunction induced by iron toxicity. PLOS ONE, 9(1), 
pp.1-15.

Stroud, J.C. et al., 2012. Toxic fibrillar oligomers of amyloid- have cross- structure. Proceedings of the National Academy of Sciences, 109(20), pp.7717-7722.

Surowka, A.D. et al., 2016. Combined in-situ imaging of structural organization and elemental composition of substantia nigra neurons in the elderly. Talanta. Available at: http://linkinghub.elsevier.com/retrieve/pii/S0039914016305896.

Sy, H.N. et al., 2010. MPTP-induced dopaminergic degeneration and deficits in object recognition in rats are accompanied by neuroinflammation in the hippocampus. Pharmacology Biochemistry and Behavior, 95(2), pp.158-165. Available at: http://dx.doi.org/10.1016/j.pbb.2009.12.020.

Szczerbowska-Boruchowska, M., Krygowska-Wajs, A. \& Adamek, D., 2012. Elemental micro-imaging and quantification of human substantia nigra using synchrotron radiation based $x$-ray fluorescence--in relation to Parkinson's disease. Journal of physics. Condensed matter: an Institute of Physics journal, 24(24), p.244104. Available at: http://www.ncbi.nlm.nih.gov/pubmed/22595486.

Tagliafierro, L. \& Chiba-Falek, O., 2016. Up-regulation of SNCA gene expression: implications to synucleinopathies. Neurogenetics, pp.1-13. Available at: http://dx.doi.org/10.1007/s10048-016-0478-0.

Tatenhorst, L. et al., 2016. Fasudil attenuates aggregation of $\alpha$-synuclein in models of Parkinson's disease. Acta neuropathologica communications, 4(1), p.39. Available at: http://www.ncbi.nlm.nih.gov/pubmed/27101974.

Tatenhorst, L. et al., 2014. Rho Kinase Inhibition by Fasudil in the Striatal 6Hydroxydopamine Lesion Mouse Model of Parkinson Disease. Journal of neuropathology and experimental neurology, 73(8), pp.770-779. Available at: http://www.ncbi.nlm.nih.gov/pubmed/25003236.

Tofaris, G.K. \& Spillantini, M.G., 2007. Physiological and pathological properties of alphasynuclein. Cellular and molecular life sciences: CMLS, 64(17), pp.2194-201. Available at: http://www.ncbi.nlm.nih.gov/pubmed/17605001.

Tönges, L. et al., 2012. Inhibition of rho kinase enhances survival of dopaminergic 
neurons and attenuates axonal loss in a mouse model of Parkinson's disease. Brain, 135, pp.3355-3370.

Trist, B.G. et al., 2016. SOD1 aggregation: A pathological link between Parkinson's disease and amyotrophic lateral sclerosis? In Mov Disord. Berlin.

Tsika, E. et al., 2010. Distinct Region-Specific -Synuclein Oligomers in A53T Transgenic Mice: Implications for Neurodegeneration. Journal of Neuroscience, 30(9), pp.3409-3418.

Available

at:

http://www.jneurosci.org/cgi/doi/10.1523/JNEUROSCI.4977-09.2010.

Tyedmers, J., Mogk, A. \& Bukau, B., 2010. Cellular strategies for controlling protein aggregation. Nature reviews. Molecular cell biology, 11(11), pp.777-788. Available at: http://dx.doi.org/10.1038/nrm2993.

Uchihara, T., 2007. Silver diagnosis in neuropathology: Principles, practice and revised interpretation. Acta Neuropathologica, 113(5), pp.483-499.

Uversky, V.N., Li, J. \& Fink, A.L., 2001. Metal-triggered Structural Transformations, Aggregation, and Fibrillation of Human -Synuclein: A POSSIBLE MOLECULAR LINK BETWEEN PARKINSON'S DISEASE AND HEAVY METAL EXPOSURE. Journal of Biological Chemistry, 276(47), pp.44284-44296. Available at: http://www.jbc.org/cgi/doi/10.1074/jbc.M105343200.

Verma, H.R., 2007. X-ray Fluorescence (XRF) and Particle-Induced X-ray Emission (PIXE). Atomic and Nuclear Analytical Methods, pp.1-90. Available at: http://dx.doi.org/10.1007/978-3-540-30279-7_1.

Wang, C. et al., 2011. Redox reactions of the $\alpha$-synuclein-Cu2+ complex and their effects on neuronal cell viability. Biochemistry, 49(37), pp.323-43.

Weinhausen, B. et al., 2012. X-ray nano-diffraction on cytoskeletal networks. New Journal of Physics, 14, p.85013.

Wobrauschek, P., Streli, C. \& Selin Lindgren, E., 2010. Energy Dispersive, X-Ray Fluorescence Analysis X-Ray Spectrometry. Encyclopedia of Analytical Chemistry.

Workman, D.G. et al., 2015. Protection from neurodegeneration in the 6hydroxydopamine (6-OHDA) model of Parkinson's with novel 1-hydroxypyridin-2- 
one metal chelators. Metallomics, 7, pp.867-876. Available at: http://xlink.rsc.org/?DOI=C4MT00326H.

Wright Willis, A. et al., 2010. Geographic and ethnic variation in Parkinson disease: A population-based study of us medicare beneficiaries. Neuroepidemiology, 34(3), pp.143-151.

Yamaguchi, H. et al., 1990. Acta Neuropatholosm. , pp.569-572.

Youdim, M.B.H., Stephenson, G. \& Ben Shachar, D., 2004. Ironing iron out in Parkinson's disease and other neurodegenerative diseases with iron chelators: a lesson from 6hydroxydopamine and iron chelators, desferal and VK-28. Annals of the New York Academy of Sciences, 1012, pp.306-25. Available at: http://www.ncbi.nIm.nih.gov/pubmed/15105275.

Zecca, L. et al., 1996. Interaction of neuromelanin and iron in substantia nigra and other areas of human brain. Neuroscience, 73(2), pp.407-415.

Zhou, M. et al., 2015. Gait analysis in three different 6-hydroxydopamine rat models of Parkinson's disease. Neuroscience Letters, 584, pp.184-189. Available at: http://dx.doi.org/10.1016/j.neulet.2014.10.032. 


\section{Acknowledgments}

(Ringraziamenti)

My first big "thank you" goes to Prof. Dr. Paul Lingor for allowing me to do my thesis in his group. Thank you for giving me the freedom to work in an independent manner while supporting and guiding me during this fascinating travel into science. I've learnt a lot from you as a person and as a scientist.

I thank Prof. Dr. Mathias Bähr for giving me the opportunity to work in the Neurology department and being part of my extended examination board.

I want to express my gratitude to Prof. Dr. Tim Salditt for being my second supervisor, for the cooperation, the helpful suggestions and discussions throughout my PhD.

I thank prof. Dr. Tiago Outeiro for being part of my thesis committee and for his valuable comments during the meetings. Furthermore I want to thank Dr. Sebastian Kügler and Dr. Kamin Dean for being members of my extended examination board and for agreeing to evaluate my work and attend my PhD defense.

Special thanks go to all the people in the lab. A big "thank you" to Lisa, Vivian and Sabine. Thanks a lot for being so helpful, for giving me excellent technical support and for sharing some of the tricks you gained through your experience.

I want to thank also Dr. Lars Tatenhorst for the help with my mice and for the helpful discussions. Thanks to Anna who helped me in becoming a bit better organized than I was 3 years ago. Thanks to Kim that made me learn a lot of "unconventional" German (Tschüssykowsky!!). Thanks to Jiannan that gave me so much affection. Thanks to Vinicius who had always a nice word for me. Thanks to Lucas and Karina, keep on singing and playing, please, you are so impressive. Thanks to Björn, for the time spent together while lysing cells for WB at the opposite site of the bench. Thanks to all the awesome AG Lingor group, you were like a family to me. Thanks to all the people in the Waldweg for all the help you gave to this crazy Italian.

I cannot forget the people at the IRP, it was cool to be a "visiting scholar" in your department, it's a very different way of thinking compared to a biology lab but 
it's in some ways complementary and it was very good for me. Special thanks to JanDavid and Mareike, for all your help during the beamtime and especially J-D for the awesome work done for the data analysis.

Thanks to Richard, Asuncion and Stefan of the CENBG. It was very nice staying in Gradignan doing experiments with you!

Special thanks to my incredible housemates. Thank you Goran and Silvia for being so supportive with me. When I had a hard day in the lab I could always chitchat with you and I knew was not alone. This meant a lot for me. Thank you Irena. More radler for everyone. Grazie a Rita e Elisa. Italians know it better!

Ora i ringraziamenti in italiano. Grazie innanzitutto ai miei genitori per avermi fatto venir qui a vivere una delle più belle avventure della mia vita. Non è stato facile per voi, lo so, e non è stato facile per me avervi lontano. Ma grazie per il vostro supporto che non mi avete mai fatto mancare. Un grazie anche alla nonna e alla zia. Non sono mai uscita con "chi non conoscevo".

Grazie sentitissimo ad Andrea. Lo sai quanto sei speciale per me, quanto il tuo supporto mi sia stato indispensabile. Tu sei indispensabile per me. Grazie per ricordarmi che andare in vacanza e rilassarmi un po' può farmi bene.

Grazie ad Alex per essere esattamente come sei. Mi manchi un sacco ma trovi sempre il modo per ricordarmi che l'amicizia vera va al di là dei km di distanza.

Grazie a Laura. Sei una persona speciale e anche se non ci vediamo spesso, so che su di te posso sempre contare. Mi dispiace ancora non poter essere stata presente al tuo dottorato.

Grazie a Lucia. Sei una fantastica amica nerd. Ti voglio bene sciaparella.

Grazie a tutti i miei amici urbinati, vicini e lontani. Vi porto sempre con me. 


\section{Publications}

$\alpha$-Synuclein Over-Expression Induces Increased Iron Accumulation and Redistribution in Iron-Exposed Neurons.

Ortega R, Carmona A, Roudeau S, Perrin L, Dučić T, Carboni E, Bohic S, Cloetens P, Lingor $\mathrm{P}$.

Mol Neurobiol. 2016 Apr;53(3):1925-34. doi: 10.1007/s12035-015-9146-x.

\section{Alpha-Synuclein Regulates Neuronal Levels of Manganese and Calcium.}

Dučić T*, Carboni E* , Lai B, Chen S, Michalke B, Lázaro DF, Outeiro TF, Bähr M, Barski E, Lingor $P$.

ACS Chem Neurosci. 2015 Oct 21;6(10):1769-79. doi: 10.1021/acschemneuro.5b00093.

* Authors contributed equally to this work

Insights on the interaction of alpha-synuclein and metals in the pathophysiology of Parkinson's disease.

Carboni $E$, Lingor $P$.

Metallomics. 2015 Mar;7(3):395-404. doi: 10.1039/c4mt00339j. Review.

Recent advances in synthesis, characterization of hydroxyapatite/polyurethane composites and study of their biocompatible properties.

Popescu LM, Piticescu RM, Antonelli A, Rusti CF, Carboni E, Sfara C, Magnani M, Badilita V, Vasile E, Trusca R, Buruiana T.

J Mater Sci Mater Med. 2013 Nov;24(11):2491-503. doi: 10.1007/s10856-013-5005-3. 


\section{Curriculum Vitae}

\section{Personal details}

\begin{tabular}{|c|c|}
\hline Name & Eleonora Carboni \\
\hline \multirow[t]{2}{*}{ Address } & Geiststr. 3c \\
\hline & 37073 Göttingen \\
\hline Email & eleonora.carboni@med.uni-goettingen.de \\
\hline Date and place of birth & $21^{\text {st }}$ August 1986 in Urbino, -PU- Italy \\
\hline Nationality & Italian \\
\hline
\end{tabular}

\section{Education}

January 2013 -

October 2016

October 2008 -

March 2011

October 2005 -

September 2008

June 2005

\section{PhD Thesis Georg-August-University, Göttingen}

University Medicine, Department of Neurology

GGNB Program "Molecular biology of the brain"

"Visualization of aggregating alpha-synuclein and its interaction with iron"

Supervisor: Prof. Dr. Paul Lingor

\section{Master of Science Alma Mater Studiorum, Bologna}

M.Sc. Program "Pharmaceutical Biotechnology"

Master thesis at the Prion Lab, S.I.S.S.A., Trieste, Italy

"full length and truncated murine prion proteins: cloning, expression and characterization of two chimeric proteins for single molecule spectroscopy studies"

Supervisor: Prof. Dr. Giuseppe Legname

\section{Bachelor of Science Alma Mater Studiorum, Bologna}

B.Sc. Program "Biotechnology"

Master thesis at the Analytical \& Bioanalytical Chemistry dept., Alma Mater Studiorum, Bologna, Italy

"Clonaggio e mutagenesi della fotoproteina equorina per la produzione di nuovi marcatori bioluminescenti per applicazioni bioanalitiche"

Supervisor: Prof. Dr. Aldo Roda

Istituto tecnico Industriale Statale "E. Mattei", Urbino

High school diploma 